Supporting Information

\title{
Synthesis of Trifluoromethylated Tetrasubstituted Allenes via Palladium-catalyzed Carbene Transfer Reaction
}

\author{
Chao Pei, Zhen Yang and Rene M. Koenigs* \\ rene.koenigs@rwth-aachen.de \\ RWTH Aachen University \\ Institute of Organic Chemistry \\ Landoltweg 1, D-52074 Aachen, Germany
}

Table of Contents

General Information

Important Safety Note

Experimental Procedures

Reaction Optimization

Physical Data

References

S16

Spectra

S17 


\section{General Information}

Unless otherwise noted, all commercially available compounds were used as provided without further purification. Chemicals used in this manuscript were purchased from Sigma Aldrich, Alfa Aesar, Fluorochem and Carl Roth. Solvents used in reactions were p.A. grade. All reactions were performed under argon using degassed solvents. Solvents for chromatography were technical grade and distilled prior to use. Analytical thin-layer chromatography (TLC) was performed on Macherey-Nagel silica gel aluminium plates with F-254 indicator, visualised by irradiation with UV light. Column chromatography was performed using silica gel Merck 60 (particle size 0.063 $-0.2 \mathrm{~mm})$. Solvent mixtures are understood as volume/volume.

${ }^{1} \mathrm{H}$ NMR, ${ }^{19} \mathrm{~F}$ NMR and ${ }^{13} \mathrm{C}$ NMR were recorded on a Varian AV600/AV400, an Agilent DD2 400 Bruker or Avance III $300 \mathrm{MHz}$ NMR spectrometer in $\mathrm{CDCl}_{3}$. Data are reported in the following order: chemical shift $(\delta)$ in ppm; multiplicities are indicated br (broadened singlet), s (singlet), d (doublet), t (triplet), q (quartet), m (multiplet); coupling constants $(J)$ are in Hertz $(\mathrm{Hz})$.

HRMS data were recorded on a ThermoFisher Scientific LTQ Orbitrap XL using ESI ionization or on a Finnigan MAT 95 using EI ionization at $70 \mathrm{eV}$. Reactions were heated with in an aluminum block.

\section{Important Safety Note}

Handling of diazo compounds should only be done in a well-ventilated fume cupboard using an additional blast shield. No incidents occurred handling of diazoalkanes during the preparation of this manuscript, yet the reader should be aware of carcinogenicity and explosiveness of the herein described diazo compounds. General safety precautions when working with diazomethane and its derivatives should be followed. Any reactions described in this manuscript should not be performed without strict risk assessment and proper safety precautions. 


\section{Experimental Procedures}

\section{General procedure for preparation of 2,2-diarylvinyl halides (GP-I)}

Step 1: A round-bottom flask was charged with methyltriphenylphosphonium bromide (3.56 g, 2.0 equiv.) and $t$ BuOK (1.12 g, 2.0 equiv.), dried THF $(15 \mathrm{~mL})$ was added under argon at $0{ }^{\circ} \mathrm{C}$ and stirred for 1 hour. After the mixture was cooled again to $0{ }^{\circ} \mathrm{C}, 2$,2-diaryl ketone ( $5 \mathrm{mmol}, 1.0$ equiv.) in THF ( $\left.5 \mathrm{~mL}\right)$ was added dropwise and stirred for $12 \mathrm{~h}$. The reaction was quenched with saturated $\mathrm{NH}_{4} \mathrm{Cl}$ (aq.) and extracted with ethyl acetate. The combined organic layer was dried over $\mathrm{MgSO}_{4}$, concentrated in vacuo and the residue was purified by column chromatography using $n$-hexane : ethyl acetate as eluent to afford the 1,1-diarylethylenes.

Step 2: This step was done by the literature procedure. ${ }^{1}$ To a suspension of 1,1-diarylethylenes $(3 \mathrm{mmol}, 1.0$ equiv.) in AcOH (10 mL) was added NBS (534 mg, 1.0 equiv.) or NCS (400 mg, 1.0 equiv.). The resulting mixture was stirred in a pre-heated oil bath at $70{ }^{\circ} \mathrm{C}$ for $4 \mathrm{~h}$. After cooling down to room temperature naturally, the reaction was neutralized by slowly adding $\mathrm{NaOH} / \mathrm{NaHCO}_{3}(1: 1)$ and extracted with ethyl acetate. The combined organic layers were dried over $\mathrm{MgSO}_{4}$ and concentrated in vacuo. The product was purified by column chromatography using $n$-hexane : ethyl acetate as eluent to afford the corresponding 2,2-diarylvinyl bromides or 2,2-diarylvinyl chlorides.

9a-d, ${ }^{1} 9 \mathbf{e},{ }^{2} 9 \mathbf{f},{ }^{3} 9 \mathrm{~g},{ }^{2} 9 \mathbf{h},{ }^{1} 9 \mathbf{j},{ }^{1} 9 \mathbf{k},{ }^{1} 9 \mathrm{~m},{ }^{1} 9 \mathbf{n},{ }^{1} 9 \mathbf{p},{ }^{1} 9 \mathbf{r},{ }^{2}{ }^{13}{ }^{1}$ were reported in the indicated references

General procedure for the Pd-catalyzed carbene transfer reactions of 2,2-diarylvinyl halides (GP-II)

In a test tube, the $\mathrm{Pd}(\mathrm{OAc})_{2}(4.5 \mathrm{mg}, 10 \mathrm{~mol} \%), \mathrm{PPh}_{3}(7.9 \mathrm{mg}, 15 \mathrm{~mol} \%)$ and $\mathrm{K}_{2} \mathrm{CO}_{3}(41.4 \mathrm{mg}, 1.5$ equiv.) were dissolved in $1.0 \mathrm{~mL}$ THF under argon and stirred in a pre-heated oil bath at $60{ }^{\circ} \mathrm{C}$ for 15 min. 2,2-Diarylvinyl halides ( $0.2 \mathrm{mmol}, 1.0$ equiv.) and trifluorodiazoalkanes (1.5 equiv.) in THF (1.5 mL) was added and stirred at $60{ }^{\circ} \mathrm{C}$ for $12 \mathrm{~h}$. The product was purified by column chromatography using $n$-hexane : ethyl acetate.

\section{General procedure for the TfOH-catalyzed Friedel-Crafts alkylation of allene (GP-III)}

To a suspension of trifluoromethylated allene $\mathbf{1 2 b}(72.8 \mathrm{mg}, 0.2 \mathrm{mmol})$ in $\mathrm{CH}_{2} \mathrm{Cl}_{2}(2 \mathrm{~mL})$ was added TfOH $(18$ $\mu \mathrm{L}, 0.15 \mathrm{mmol}$ ) at room temperature. The resulting mixture was stirred at room temperature for $2 \mathrm{~h}$. Then the mixture was poured into ice water $(20 \mathrm{~mL})$ and extracted with $\mathrm{CH}_{2} \mathrm{Cl}_{2}$. The combined organic layers were washed with saturated $\mathrm{NaHCO}_{3}$ (aq.), dried over $\mathrm{MgSO}_{4}$ and concentrated in vacuo. The residue was purified by column chromatography using $n$-hexane as eluent to afford the $1 H$-indene $\mathbf{1 6}$ (71 $\mathrm{mg}, 97 \%$ yield).

\section{General procedure for the $\mathrm{Yb}(\mathrm{OTf})_{3}$-catalyzed Friedel-Crafts alkylation of allene (GP-IV)}

To a test tube were successively added $\mathbf{1 2 b}(72.8 \mathrm{mg}, 0.2 \mathrm{mmol}), \mathrm{Yb}(\mathrm{OTf})_{3}(6.2 \mathrm{mg}, 5 \mathrm{~mol} \%)$ and $\mathrm{CH}_{3} \mathrm{NO}_{2}(2$ $\mathrm{mL}$ ). The resulting mixture was stirred in a pre-heated oil bath at $80{ }^{\circ} \mathrm{C}$ for $12 \mathrm{~h}$. On completion, the reaction mixture was concentrated in vacuo and the product was directly purified by column chromatography using $n$ hexane as eluent to afford the $\mathbf{1 6}$ (55 $\mathrm{mg}, 75 \%$ yield) and $\mathbf{1 7}$ (14 $\mathrm{mg}, 19 \%$ yield).

\section{Gram-scale reaction}

In a test tube, the $\mathrm{Pd}(\mathrm{OAc})_{2}(39.3 \mathrm{mg}, 5 \mathrm{~mol} \%), \mathrm{PPh}_{3}(68.9 \mathrm{mg}, 7.5 \mathrm{~mol} \%)$ and $\mathrm{K}_{2} \mathrm{CO}_{3}(724.5 \mathrm{mg}, 1.5$ equiv.) were dissolved in $15 \mathrm{~mL}$ THF under argon and stirred in a pre-heated oil bath at $60{ }^{\circ} \mathrm{C}$ for $15 \mathrm{~min}$. 4,4'-(2Bromoethene-1,1-diyl)bis(methylbenzene) (1.00 g, $3.5 \mathrm{mmol}, 1.0$ equiv.) and trifluorodiazoalkane (976.5 mg, 1.5 equiv.) in THF (20 mL) was added via syringe and stirred at $60^{\circ} \mathrm{C}$ for $12 \mathrm{~h}$. The crude product was purified by column chromatography using $n$-hexane as eluent; $90 \%$ yield (1.14 g). 


\section{Reaction Optimization}

\begin{tabular}{|c|c|c|c|c|}
\hline $\begin{array}{l}0.2 \mathrm{mn} \\
9 \mathbf{9 a}\end{array}$ & $\begin{array}{r}0.1 \\
0.3 n \\
4\end{array}$ & $\begin{array}{ll} & \mathrm{Pd}( \\
& \\
\mathrm{CF}_{3} & \\
& \end{array}$ & $\begin{array}{l}\frac{f_{2}(10 \mathrm{~mol} \%)}{(15 \mathrm{~mol} \%)} \\
\stackrel{\text { solvent }}{\text { overnight }}\end{array}$ & ${ }^{\mathrm{Ph}} \prod_{i} \mathrm{CF}_{3}$ \\
\hline entry ${ }^{[a]}$ & ligand & base & solvent & yield $\%$ \\
\hline 1 & dpph & $\mathrm{CsOAc}$ & THF & 15 \\
\hline 2 & dpph & $\mathrm{KOH}$ & THF & 9 \\
\hline 3 & dpph & $\mathrm{K}_{2} \mathrm{CO}_{3}$ & THF & 72 \\
\hline 4 & dpph & $\mathrm{Li}_{2} \mathrm{CO}_{3}$ & THF & 8 \\
\hline 5 & dpph & $\mathrm{LiOH}$ & THF & 3 \\
\hline 6 & dpph & $\mathrm{NaOAc}$ & THF & 5 \\
\hline 7 & dpph & $\mathrm{NEt}_{3}$ & THF & 8 \\
\hline 8 & dppb & $\mathrm{K}_{2} \mathrm{CO}_{3}$ & THF & 74 \\
\hline 9 & dppe & $\mathrm{K}_{2} \mathrm{CO}_{3}$ & THF & 76 \\
\hline 10 & dppf & $\mathrm{K}_{2} \mathrm{CO}_{3}$ & THF & 71 \\
\hline 11 & $r a c$-BINAP & $\mathrm{K}_{2} \mathrm{CO}_{3}$ & THF & 5 \\
\hline 12 & $\mathrm{PAd}_{2} n \mathrm{Bu}$ & $\mathrm{K}_{2} \mathrm{CO}_{3}$ & THF & 72 \\
\hline 13 & DavePhos & $\mathrm{K}_{2} \mathrm{CO}_{3}$ & THF & 4 \\
\hline 14 & SPhos & $\mathrm{K}_{2} \mathrm{CO}_{3}$ & THF & traces \\
\hline 15 & XPhos & $\mathrm{K}_{2} \mathrm{CO}_{3}$ & THF & traces \\
\hline 16 & RuPhos & $\mathrm{K}_{2} \mathrm{CO}_{3}$ & THF & traces \\
\hline 17 & PFur $_{3}$ & $\mathrm{~K}_{2} \mathrm{CO}_{3}$ & THF & 67 \\
\hline 18 & $\mathrm{PoTol}_{3}$ & $\mathrm{~K}_{2} \mathrm{CO}_{3}$ & THF & 6 \\
\hline 19 & $\mathrm{PPh}_{3}$ & $\mathrm{~K}_{2} \mathrm{CO}_{3}$ & THF & $95(92)^{[b]}$ \\
\hline 20 & $\mathrm{PPh}_{3}$ & $\mathrm{~K}_{2} \mathrm{CO}_{3}$ & 1,2-DCE & 42 \\
\hline 21 & $\mathrm{PPh}_{3}$ & $\mathrm{~K}_{2} \mathrm{CO}_{3}$ & toluene & 56 \\
\hline 22 & $\mathrm{PPh}_{3}$ & $\mathrm{~K}_{2} \mathrm{CO}_{3}$ & 1,4-dioxane & 92 \\
\hline 23 & $\mathrm{PPh}_{3}$ & $\mathrm{~K}_{2} \mathrm{CO}_{3}$ & DMF & 19 \\
\hline $24^{[\mathrm{c}]}$ & - & $\mathrm{K}_{2} \mathrm{CO}_{3}$ & THF & traces \\
\hline $25^{[\mathrm{d}]}$ & $\mathrm{PPh}_{3}$ & $\mathrm{~K}_{2} \mathrm{CO}_{3}$ & THF & 8 \\
\hline
\end{tabular}

[a] Reaction condition: $0.2 \mathrm{mmol} 9 \mathrm{a}, 0.3 \mathrm{mmol} 4 \mathbf{a}, 10 \mathrm{~mol} \% \mathrm{Pd}(\mathrm{OAc})_{2}$, $15 \mathrm{~mol} \%$ ligand and $0.3 \mathrm{mmol}$ base were dissolved in $2.5 \mathrm{~mL}$ solvent and stirred at $60^{\circ} \mathrm{C}$ for $12 \mathrm{~h}$ under Argon atmosphere. The yield was determined by ${ }^{19} \mathrm{~F}-\mathrm{NMR}$ of the reaction crude. The internal standard is $\mathrm{PhCF}_{3}$. ${ }^{[\mathrm{b}]}$ Yield refers to isolated products. ${ }^{[\mathrm{c}]}$ Without ligand. ${ }^{[\mathrm{d}]} 5$ mol\% $\mathrm{Pd}_{2}(\mathrm{dba})_{3}$ was used. 


\section{Physical Data}

\section{1-Bromo-4-(2-bromo-1-phenylvinyl)benzene (9i)}<smiles>BrC=C(c1ccccc1)c1ccc(Br)cc1</smiles>

The titled compound (9i) was synthesized according to the general procedure GP-I, and was obtained after silica gel column chromatography using $n$-hexane as colorless oil $(85 \%, 860 \mathrm{mg}$, isomeric ratio: 5:3).

${ }^{1}$ H NMR $(600 \mathrm{MHz}$, Chloroform- $d): \delta=7.56(\mathrm{~d}, J=8.1 \mathrm{~Hz}, 1.27 \mathrm{H}), 7.48-7.37(\mathrm{~m}, 1.91 \mathrm{H}), 7.35-7.28(\mathrm{~m}$, $2.74 \mathrm{H}), 7.24-7.17(\mathrm{~m}, 2.60 \mathrm{H}), 7.10(\mathrm{~d}, J=8.3 \mathrm{~Hz}, 0.76 \mathrm{H}), 6.80(\mathrm{~s}, 1 \mathrm{H}) \mathrm{ppm}$.

${ }^{13}$ C NMR (151 MHz, Chloroform- $d$ ): $\delta=145.9,140.3,139.7,138.6,138.0,131.7,131.6,131.5,129.7,129.2$, $128.6,128.4,128.3,127.7,122.4,122.3,105.8,105.7 \mathrm{ppm}$.

IR (KBr): 3431, 3059, 2923, 2654, 2325, 2085, 1953, 1908, 1790, 1658, 1623, 1590, 1483, 1442, 1388, 1321 , $1260,1213,1175,1151,1098,1069,1007,952,912,825,795,750,693 \mathrm{~cm}^{-1}$.

HRMS (APCI) $m / z:[\mathrm{M}+\mathrm{H}]^{+}$mass found: 336.9222, mass calculated for $\mathrm{C}_{14} \mathrm{H}_{11} \mathrm{Br}_{2}{ }^{+}: 336.9220$.

1-(2-Bromo-1-phenylvinyl)-3-methylbenzene (91)<smiles>Cc1cccc(/C(=C\Br)c2ccccc2)c1</smiles>

The titled compound (91) was synthesized according to the general procedure GP-I, and was obtained after silica gel column chromatography using $n$-hexane as colorless oil $(90 \%, 732 \mathrm{mg}$, isomeric ratio: 1:1).

${ }^{1}$ H NMR $(600 \mathrm{MHz}$, Chloroform- $d)$ : $\delta=7.45(\mathrm{t}, J=7.2 \mathrm{~Hz}, 2 \mathrm{H}), 7.42-7.39(\mathrm{~m}, 1 \mathrm{H}), 7.39-7.30(\mathrm{~m}, 6 \mathrm{H}), 7.29$ $-7.20(\mathrm{~m}, 4 \mathrm{H}), 7.19-7.13(\mathrm{~m}, 3 \mathrm{H}), 7.09(\mathrm{~s}, 1 \mathrm{H}), 7.06(\mathrm{~d}, J=7.8 \mathrm{~Hz}, 1 \mathrm{H}), 6.80(\mathrm{~s}, 2 \mathrm{H}), 2.42(\mathrm{~s}, 3 \mathrm{H}), 2.36(\mathrm{~s}, 3 \mathrm{H})$ ppm.

${ }^{13}$ C NMR (151 MHz, Chloroform- $d$ ): $\delta=147.1,147.0,140.9,140.8,139.2,139.1,138.1,137.9,130.2,129.7$, $129.0,128.8,128.5,128.4,128.39,128.31,128.2,128.1,128.0,127.7,126.8,124.9,105.17,105.13,21.57,21.51$ ppm.

IR (KBr): 3393, 3059, 3028, 2920, 2859, 2661, 2326, 2085, 2000, 1942, 1880, 1801, 1593, 1490, 1442, 1379 , 1324, 1219, 1184, 1074, 1031, 1000, 954, 911, 884, 841, 773, 728, $696 \mathrm{~cm}^{-1}$.

HRMS (APCI) $m / z:[\mathrm{M}+\mathrm{H}]^{+}$mass found: 273.0273, mass calculated for $\mathrm{C}_{15} \mathrm{H}_{14} \mathrm{Br}^{+}: 273.0278$.

\section{1-(2-Bromo-1-phenylvinyl)-2-chlorobenzene (9o)}<smiles>Clc1ccccc1/C(=C\Br)c1ccccc1</smiles>

The titled compound (9o) was synthesized according to the general procedure GP-I, and was obtained after silica gel column chromatography using $n$-hexane as colorless oil $(83 \%, 725 \mathrm{mg}$, isomeric ratio: 3:1).

${ }^{1}$ H NMR (600 MHz, Chloroform- $d$ ): $\delta=7.77-7.21(\mathrm{~m}, 9 \mathrm{H}), 7.04(\mathrm{~s}, 0.75 \mathrm{H}), 6.65(\mathrm{~s}, 0.25 \mathrm{H}) \mathrm{ppm}$.

${ }^{13}$ C NMR (151 MHz, Chloroform- $d$ ): $\delta=144.7,144.0,140.1,138.5,138.2,137.9,133.35,133.31,131.5,131.2$, $130.2,130.0,129.5,129.3,128.7,128.3,128.1,128.0,127.0,126.8,126.6,108.08,108.07,107.7$ ppm.

IR (KBr): 3856, 3388, 3063, 2923, 2664, 2324, 2111, 1995, 1882, 1808, 1594, 1492, 1470, 1437, 1324, 1258 , $1216,1156,1126,1054,1033,912,865,809,750,692 \mathrm{~cm}^{-1}$.

HRMS (APCI) $m / z:[\mathrm{M}+\mathrm{H}]^{+}$mass found: 292.9727, mass calculated for $\mathrm{C}_{14} \mathrm{H}_{11} \mathrm{BrCl}^{+}: 292.9731$. 
<smiles>Br/C=C(/c1ccccc1)c1cccc2ccccc12</smiles>

The titled compound (9q) was synthesized according to the general procedure GP-I, and was obtained after silica gel column chromatography using $n$-hexane as white solid $(92 \%, 850 \mathrm{mg}$, isomeric ratio: $2: 1)$.

${ }^{1}$ H NMR $(600 \mathrm{MHz}$, Chloroform- $d): \delta=7.97-7.94(\mathrm{~m}, 2.5 \mathrm{H}), 7.89(\mathrm{~d}, J=7.8 \mathrm{~Hz}, 1 \mathrm{H}), 7.83(\mathrm{~d}, J=8.4 \mathrm{~Hz}, 1 \mathrm{H})$, $7.64-7.56(\mathrm{~m}, 2 \mathrm{H}), 7.55-7.42(\mathrm{~m}, 5 \mathrm{H}), 7.38(\mathrm{t}, J=7.5 \mathrm{~Hz}, 1 \mathrm{H}), 7.34-7.28(\mathrm{~m}, 5.5 \mathrm{H}), 7.22(\mathrm{~s}, 1 \mathrm{H}), 6.71(\mathrm{~s}$, $0.5 \mathrm{H}) \mathrm{ppm}$.

${ }^{13}$ C NMR (151 MHz, Chloroform- $d$ ): $\delta=145.6,145.0,139.9,139.1,139.0,137.0,133.98,133.90,131.7,131.0$, $129.1,128.8,128.7,128.5,128.4,128.3,128.27,128.22,128.1,127.7,127.3,126.6,126.5,126.4,126.1,126.0$, $125.9,125.6,125.3,108.1,106.5 \mathrm{ppm}$.

IR (KBr): 3847, 3057, 2922, 2664, 2321, 2113, 1995, 1880, 1811, 1590, 1494, 1441, 1393, 1317, 1253, 1215 , $1182,1154,1075,1016,969,917,864,771,693 \mathrm{~cm}^{-1}$.

HRMS (APCI) $m / z:[\mathrm{M}+\mathrm{H}]^{+}$mass found: 309.0281, mass calculated for $\mathrm{C}_{18} \mathrm{H}_{14} \mathrm{Br}^{+}: 309.0273$.

\section{5-(Bromomethylene)-10,11-dihydro-5H-dibenzo[ $a, d][7]$ annulene $(9 \mathrm{~s})$}<smiles>BrC=C1c2ccccc2CCc2ccccc21</smiles>

The titled compound (9s) was synthesized according to the general procedure GP-I, and was obtained after silica gel column chromatography using $n$-hexane as white solid $(91 \%, 772 \mathrm{mg})$.

${ }^{1}$ H NMR (400 MHz, Chloroform- $d$ ): $\delta=7.39-7.33(\mathrm{~m}, 1 \mathrm{H}), 7.31-7.15(\mathrm{~m}, 6 \mathrm{H}), 7.10(\mathrm{~d}, J=7.4 \mathrm{~Hz}, 1 \mathrm{H}), 6.65$ (s, 1H), $3.50-3.24(\mathrm{~m}, 2 \mathrm{H}), 3.03-2.73(\mathrm{~m}, 2 \mathrm{H}) \mathrm{ppm}$.

${ }^{13}$ C NMR (101 MHz, Chloroform- $d$ ): $\delta=147.7,139.1,138.8,138.5,137.7,130.3,128.6,128.45,128.40,128.3$, $128.2,126.3,125.9,106.1,33.4,31.9 \mathrm{ppm}$.

IR (KBr): 3855, 3362, 3064, 2911, 2854, 2656, 2325, 2185, 2083, 1924, 1819, 1737, 1602, 1482, 1442, 1357 , $1322,1257,1221,1203,1156,1087,1032,965,904,810,750,697 \mathrm{~cm}^{-1}$.

HRMS (APCI) $m / z:[\mathrm{M}+\mathrm{H}]^{+}$mass found: 285.0277, mass calculated for $\mathrm{C}_{16} \mathrm{H}_{14} \mathrm{Br}^{+}: 285.0273$.

5-(Bromomethylene)-5H-dibenzo[a,d][7]annulene (9t)<smiles>BrC=C1c2ccccc2C=Cc2ccccc21</smiles>

The titled compound (9t) was synthesized according to the general procedure GP-I, and was obtained after silica gel column chromatography using $n$-hexane as white solid $(85 \%, 722 \mathrm{mg})$.

${ }^{1}$ H NMR $(300 \mathrm{MHz}$, Chloroform- $d)$ : $\delta=7.53-7.48(\mathrm{~m}, 1 \mathrm{H}), 7.47-7.41(\mathrm{~m}, 1 \mathrm{H}), 7.41-7.30(\mathrm{~m}, 6 \mathrm{H}), 6.94(\mathrm{~d}$, $J=12.0 \mathrm{~Hz}, 1 \mathrm{H}), 6.88(\mathrm{~d}, J=12.0 \mathrm{~Hz}, 1 \mathrm{H}), 6.41(\mathrm{~d}, J=0.7 \mathrm{~Hz}, 1 \mathrm{H}) \mathrm{ppm}$.

${ }^{13}$ C NMR (75 MHz, Chloroform- $d$ ): $\delta=145.9,139.4,136.0,134.0,133.9,131.2,130.5,129.1,128.8,128.7$, 128.6, 128.1, 127.87, 127.82, 127.3, $107.8 \mathrm{ppm}$.

IR (KBr): 3857, 3550, 3057, 3014, 2922, 2854, 2321, 2113, 2018, 1913, 1836, 1742, 1603, 1552, 1484, 1432 , 1372, 1326, 1303, 1273, 1210, 1157, 1090, 1036, 986, 955, 927, 882, 857, 797, 770, $725 \mathrm{~cm}^{-1}$.

HRMS (APCI) $m / z:[\mathrm{M}+\mathrm{H}]^{+}$mass found: 283.0124, mass calculated for $\mathrm{C}_{16} \mathrm{H}_{12} \mathrm{Br}^{+}: 283.0116$. 
<smiles>FC(F)(F)C(=C(c1ccccc1)c1ccccc1)c1ccccc1</smiles>

The titled compound was synthesized according to the general procedure GP-II, and was obtained after silica gel column chromatography using $n$-hexane as colorless oil $(92 \%, 62 \mathrm{mg})$.

${ }^{1}$ H NMR $(600 \mathrm{MHz}$, Chloroform- $d): \delta=7.56(\mathrm{~d}, J=7.8 \mathrm{~Hz}, 2 \mathrm{H}), 7.44-7.37(\mathrm{~m}, 12 \mathrm{H}), 7.34(\mathrm{t}, J=7.4 \mathrm{~Hz}, 1 \mathrm{H})$ ppm.

${ }^{13}$ C NMR (151 MHz, Chloroform- $d$ ): $\delta=207.0(\mathrm{q}, J=3.9 \mathrm{~Hz}), 134.3,129.8,129.0,128.9,128.8,128.6,127.2$, $123.5(\mathrm{q}, J=275.2 \mathrm{~Hz}), 118.3,104.6(\mathrm{q}, J=34.3 \mathrm{~Hz}) \mathrm{ppm}$. Due to the overlap of aromatic carbon peaks, one carbon signal is missing.

${ }^{19}$ F NMR (565 MHz, Chloroform- $d$ ): $\delta=-59.91 \mathrm{ppm}$.

IR (KBr): 3061, 3032, 2926, 2855, 2321, 2162, 2049, 1947, 1888, 1805, 1763, 1598, 1493, 1447, 1375, 1296, $1167,1118,1031,999,928,841,763,691,663 \mathrm{~cm}^{-1}$.

HRMS (ESI) $m / z:[\mathrm{M}]^{+}$mass found: 336.1119 , mass calculated for $\mathrm{C}_{22} \mathrm{H}_{15} \mathrm{~F}_{3}{ }^{+}: 336.1120$.

4,4'-(4,4,4-Trifluoro-3-phenylbuta-1,2-diene-1,1-diyl)bis(methylbenzene) (12b)<smiles>Cc1ccc(C(=C=C(c2ccccc2)C(F)(F)F)c2ccc(C)cc2)cc1</smiles>

The titled compound was synthesized according to the general procedure GP-II, and was obtained after silica gel column chromatography using $n$-hexane as white solid $(93 \%, 68 \mathrm{mg})$.

${ }^{1}$ H NMR $(600 \mathrm{MHz}$, Chloroform- $d): \delta=7.54(\mathrm{~d}, J=7.7 \mathrm{~Hz}, 2 \mathrm{H}), 7.39(\mathrm{t}, J=7.6 \mathrm{~Hz}, 2 \mathrm{H}), 7.33(\mathrm{~d}, J=7.2 \mathrm{~Hz}$, $1 \mathrm{H}), 7.30(\mathrm{~d}, J=8.0 \mathrm{~Hz}, 4 \mathrm{H}), 7.21(\mathrm{~d}, J=7.9 \mathrm{~Hz}, 4 \mathrm{H}), 2.40(\mathrm{~s}, 6 \mathrm{H}) \mathrm{ppm}$.

${ }^{13}$ C NMR (151 MHz, Chloroform- $d$ ): $\delta=206.9(\mathrm{q}, J=3.9 \mathrm{~Hz}), 138.7,131.4,130.0,129.6,128.9,128.6,128.4$, 127.1, $123.5(\mathrm{q}, J=275.1 \mathrm{~Hz}), 118.1,104.1(\mathrm{q}, J=34.3 \mathrm{~Hz}), 21.4 \mathrm{ppm}$.

${ }^{19}$ F NMR (565 MHz, Chloroform- $d$ ): $\delta=-59.91 \mathrm{ppm}$.

IR (KBr): 3352, 3027, 2924, 2857, 2159, 2042, 1913, 1732, 1661, 1606, 1505, 1450, 1414, 1373, 1290, 1187 , $1156,1119,1031,968,928,821,761,718,696,658 \mathrm{~cm}^{-1}$.

HRMS (ESI) $m / z:[M]^{+}$mass found: 364.1435 , mass calculated for $\mathrm{C}_{24} \mathrm{H}_{19} \mathrm{~F}_{3}{ }^{+}: 364.1433$.

4,4'-(4,4,4-Trifluoro-3-phenylbuta-1,2-diene-1,1-diyl)bis(fluorobenzene) (12c)<smiles>Fc1ccc(C(=C=C(c2ccccc2)C(F)(F)F)c2ccc(F)cc2)cc1</smiles>

The titled compound was synthesized according to the general procedure GP-II, and was obtained after silica gel column chromatography using $n$-hexane as colorless oil $(91 \%, 68 \mathrm{mg})$.

${ }^{1}$ H NMR $(600 \mathrm{MHz}$, Chloroform- $d): \delta=7.53(\mathrm{~d}, J=7.7 \mathrm{~Hz}, 2 \mathrm{H}), 7.41(\mathrm{t}, J=7.7 \mathrm{~Hz}, 2 \mathrm{H}), 7.38-7.34(\mathrm{~m}, 5 \mathrm{H})$, $7.11(\mathrm{t}, J=8.5 \mathrm{~Hz}, 4 \mathrm{H}) \mathrm{ppm}$.

${ }^{13}$ C NMR (151 MHz, Chloroform- $\left.d\right): \delta=206.6(\mathrm{q}, J=4.2 \mathrm{~Hz}), 163.1(\mathrm{~d}, J=249.2 \mathrm{~Hz}), 130.4(\mathrm{~d}, J=8.3 \mathrm{~Hz})$, $130.1(\mathrm{~d}, J=3.5 \mathrm{~Hz}), 129.5,129.1,128.8,127.1,123.3(\mathrm{q}, J=275.1 \mathrm{~Hz}), 116.6,116.1(\mathrm{~d}, J=21.8 \mathrm{~Hz}), 104.8(\mathrm{q}$, $J=34.3 \mathrm{~Hz}) \mathrm{ppm}$.

${ }^{19}$ F NMR $(565 \mathrm{MHz}$, Chloroform- $d$ ): $\delta=-60.00(\mathrm{~s}, 3 \mathrm{~F}),-112.33--112.38(\mathrm{~m}, 2 \mathrm{~F}) \mathrm{ppm}$.

IR (KBr): 3340, 3063, 2926, 2157, 2029, 1895, 1767, 1653, 1601, 1504, 1418, 1370, 1296, 1230, 1162, 1120, $1014,929,836,763,721,690,659 \mathrm{~cm}^{-1}$.

HRMS (ESI) $m / z:[\mathrm{M}]^{+}$mass found: 372.0932 , mass calculated for $\mathrm{C}_{22} \mathrm{H}_{13} \mathrm{~F}_{5}{ }^{+}: 372.0931$. 
<smiles>FC(F)(F)C(=C=C(c1ccc(Cl)cc1)c1ccc(Cl)cc1)c1ccccc1</smiles>

The titled compound was synthesized according to the general procedure GP-II, and was obtained after silica gel column chromatography using $n$-hexane as colorless oil $(94 \%, 76 \mathrm{mg})$.

${ }^{1}$ H NMR $(600 \mathrm{MHz}$, Chloroform- $d): \delta=7.51(\mathrm{~d}, J=7.7 \mathrm{~Hz}, 2 \mathrm{H}), 7.42-7.35(\mathrm{~m}, 7 \mathrm{H}), 7.31(\mathrm{~d}, J=8.5 \mathrm{~Hz}, 4 \mathrm{H})$ ppm.

${ }^{13}$ C NMR (151 MHz, Chloroform- $d$ ): $\delta=206.7$ (q, $\left.J=3.9 \mathrm{~Hz}\right), 135.0,132.4,129.9,129.3,129.2,129.1,128.9$, 127.1, 123.2 (q, $J=275.4 \mathrm{~Hz}), 116.6,105.3$ (q, $J=34.3 \mathrm{~Hz}) \mathrm{ppm}$.

${ }^{19}$ F NMR (565 MHz, Chloroform- $d$ ): $\delta=-59.96$ ppm.

IR (KBr): 3333, 3064, 2926, 2856, 2163, 2033, 1902, 1771, 1653, 1592, 1489, 1409, 1369, 1296, 1168, 1123 , $1091,1013,928,909,829,765,719,692,662 \mathrm{~cm}^{-1}$.

HRMS (ESI) $m / z$ : [M] ${ }^{+}$mass found: 404.0344, mass calculated for $\mathrm{C}_{22} \mathrm{H}_{13} \mathrm{Cl}_{2} \mathrm{~F}_{3}{ }^{+}: 404.0340$.

4,4'-(4,4,4-Trifluoro-3-phenylbuta-1,2-diene-1,1-diyl)bis(methoxybenzene) (12e)<smiles>COc1ccc(C(=C=C(c2ccccc2)C(F)(F)F)c2ccc(OC)cc2)cc1</smiles>

The titled compound was synthesized according to the general procedure GP-II, and was obtained after silica gel column chromatography using $n$-hexane : ethyl acetate $=20: 1$ as colorless oil $(96 \%, 76 \mathrm{mg})$.

${ }^{1}$ H NMR $(600 \mathrm{MHz}$, Chloroform- $d): \delta=7.53(\mathrm{~d}, J=7.8 \mathrm{~Hz}, 2 \mathrm{H}), 7.38(\mathrm{t}, J=7.6 \mathrm{~Hz}, 2 \mathrm{H}), 7.36-7.30(\mathrm{~m}, 5 \mathrm{H})$, $6.93(\mathrm{~d}, J=8.7 \mathrm{~Hz}, 4 \mathrm{H}), 3.84(\mathrm{~s}, 6 \mathrm{H}) \mathrm{ppm}$.

${ }^{13}$ C NMR (151 MHz, Chloroform- $d$ ): $\delta=206.8$ (q, $\left.J=3.9 \mathrm{~Hz}\right), 160.1,130.2,130.0,128.9,128.3,127.0,126.6$, $123.5(\mathrm{q}, J=275.1 \mathrm{~Hz}), 117.6,114.3,103.9(\mathrm{q}, J=34.1 \mathrm{~Hz}), 55.5 \mathrm{ppm}$.

${ }^{19}$ F NMR (565 MHz, Chloroform- $d$ ): $\delta=-59.93 \mathrm{ppm}$.

IR (KBr): 3005, 2932, 2841, 2547, 2195, 2043, 1893, 1730, 1605, 1507, 1461, 1371, 1294, 1247, 1168, 1117 , $1031,928,834,762,729,693,658 \mathrm{~cm}^{-1}$.

HRMS (ESI) $m / z:[M]^{+}$mass found: 396.1329 , mass calculated for $\mathrm{C}_{24} \mathrm{H}_{19} \mathrm{~F}_{3} \mathrm{O}_{2}{ }^{+}: 396.1331$.

(4,4,4-Trifluoro-1-( $p$-tolyl)buta-1,2-diene-1,3-diyl)dibenzene (12f)<smiles>Cc1ccc(C(=C=C(c2ccccc2)C(F)(F)F)c2ccccc2)cc1</smiles>

The titled compound was synthesized according to the general procedure GP-II, and was obtained after silica gel column chromatography using $n$-hexane as colorless oil $(89 \%, 62 \mathrm{mg})$.

${ }^{1}$ H NMR $(600 \mathrm{MHz}$, Chloroform- $d): \delta=7.55(\mathrm{~d}, J=7.7 \mathrm{~Hz}, 2 \mathrm{H}), 7.43-7.37(\mathrm{~m}, 7 \mathrm{H}), 7.34(\mathrm{~d}, J=7.3 \mathrm{~Hz}, 1 \mathrm{H})$, $7.31(\mathrm{~d}, J=7.5 \mathrm{~Hz}, 2 \mathrm{H}), 7.22(\mathrm{~d}, J=7.8 \mathrm{~Hz}, 2 \mathrm{H}), 2.40(\mathrm{~s}, 3 \mathrm{H}) \mathrm{ppm}$.

${ }^{13}$ C NMR (151 MHz, Chloroform- $d$ ): $\delta=206.9(\mathrm{q}, J=3.7 \mathrm{~Hz}), 138.8,134.5,131.3,123.0,129.7,129.0,128.9$, $128.8,128.7,128.6,128.5,127.2,123.5$ (q, $J=275.1 \mathrm{~Hz}), 118.2,104.4$ (q, $J=34.0 \mathrm{~Hz}), 21.4 \mathrm{ppm}$.

${ }^{19}$ F NMR (565 MHz, Chloroform- $\left.d\right)$ : $\delta=-59.91 \mathrm{ppm}$.

IR (KBr): 3030, 2924, 2859, 2166, 1944, 1802, 1763, 1601, 1495, 1448, 1413, 1372, 1297, 1166, 1118, 1031 , $968,928,820,762,694,660 \mathrm{~cm}^{-1}$.

HRMS (ESI) $m / z:[\mathrm{M}]^{+}$mass found: 350.1274 , mass calculated for $\mathrm{C}_{23} \mathrm{H}_{17} \mathrm{~F}_{3}{ }^{+}: 350.1276$. 
<smiles>Fc1ccc(C(=CC(F)(F)F)c2ccccc2)cc1</smiles>

The titled compound was synthesized according to the general procedure GP-II, and was obtained after silica gel column chromatography using $n$-hexane as colorless oil $(96 \%, 68 \mathrm{mg})$.

${ }^{1} \mathrm{H}$ NMR $(600 \mathrm{MHz}$, Chloroform- $d): \delta=7.55(\mathrm{~d}, J=7.8 \mathrm{~Hz}, 2 \mathrm{H}), 7.46-7.33(\mathrm{~m}, 10 \mathrm{H}), 7.11(\mathrm{t}, J=8.5 \mathrm{~Hz}, 2 \mathrm{H})$ ppm.

${ }^{13}$ C NMR (151 MHz, Chloroform- $d$ ): $\delta=206.7$ (q, $\left.J=4.0 \mathrm{~Hz}\right), 163.0(\mathrm{~d}, J=248.6 \mathrm{~Hz}), 134.1,130.4(\mathrm{~d}, J=8.1$ $\mathrm{Hz}), 130.2(\mathrm{~d}, J=3.4 \mathrm{~Hz}), 129.5,129.0,128.9,128.8,128.6,127.0,123.3(\mathrm{q}, J=275.1 \mathrm{~Hz}), 117.4,115.9(\mathrm{~d}, J=$ $21.9 \mathrm{~Hz}), 104.6(\mathrm{q}, J=34.4 \mathrm{~Hz}) \mathrm{ppm}$. Due to the overlap of aromatic carbon peaks, one carbon signal is missing. ${ }^{19}$ F NMR (565 MHz, Chloroform- $d$ ): $\delta=-59.95$ (s, 3F), $-112.64--112.68(\mathrm{~m}, 1 \mathrm{~F}) \mathrm{ppm}$.

IR (KBr): 3062, 2926, 2168, 1947, 1893, 1766, 1600, 1503, 1448, 1413, 1372, 1296, 1231, 1165, 1120, 1031, $967,929,838,761,694,661 \mathrm{~cm}^{-1}$.

HRMS (ESI) $m / z:[\mathrm{M}]^{+}$mass found: 354.1027 , mass calculated for $\mathrm{C}_{22} \mathrm{H}_{14} \mathrm{~F}_{4}^{+}: 354.1026$.

(1-(4-Chlorophenyl)-4,4,4-trifluorobuta-1,2-diene-1,3-diyl)dibenzene (12h)<smiles>FC(F)(F)C(=Cc1ccccc1)c1ccc(Cl)cc1</smiles>

The titled compound was synthesized according to the general procedure GP-II, and was obtained after silica gel column chromatography using $n$-hexane as colorless oil $(93 \%, 69 \mathrm{mg})$.

${ }^{1}$ H NMR (600 MHz, Chloroform- $d$ ): $\delta=7.55(\mathrm{~d}, J=7.3 \mathrm{~Hz}, 2 \mathrm{H}), 7.44-7.38(\mathrm{~m}, 9 \mathrm{H}), 7.38-7.33(\mathrm{~m}, 3 \mathrm{H}) \mathrm{ppm}$. ${ }^{13}$ C NMR (151 MHz, Chloroform- $d$ ): $\delta=206.9(\mathrm{q}, J=4.1 \mathrm{~Hz}), 134.8,133.9,132.8,130.0,129.4,129.2,129.1$, $129.06,129.02,128.8,128.7,127.2,123.4(\mathrm{q}, J=275.2 \mathrm{~Hz}), 117.5,104.9(\mathrm{q}, J=34.4 \mathrm{~Hz}) \mathrm{ppm}$.

${ }^{19}$ F NMR $(565 \mathrm{MHz}$, Chloroform- $d$ ): $\delta=-59.91 \mathrm{ppm}$.

IR (KBr): 3062, 2926, 2856, 2168, 2025, 1944, 1900, 1768, 1702, 1596, 1489, 1448, 1405, 1371, 1296, 1168 , $1211,1013,966,928,830,760,730,693,661 \mathrm{~cm}^{-1}$.

HRMS (ESI) $\mathrm{m} / z:[\mathrm{M}]^{+}$mass found: 370.0729 , mass calculated for $\mathrm{C}_{22} \mathrm{H}_{14} \mathrm{ClF}_{3}{ }^{+}: 370.0730$. 
<smiles>FC(F)(F)C(=C=C(c1ccccc1)c1ccc(Br)cc1)c1ccccc1</smiles>

The titled compound was synthesized according to the general procedure GP-II, and was obtained after silica gel column chromatography using $n$-hexane as colorless oil $(69 \%, 57 \mathrm{mg})$.

${ }^{1}$ H NMR $(600 \mathrm{MHz}$, Chloroform- $d$ ): $\delta=7.55-7.50(\mathrm{~m}, 4 \mathrm{H}), 7.45-7.33(\mathrm{~m}, 8 \mathrm{H}), 7.28(\mathrm{~d}, J=8.5 \mathrm{~Hz}, 2 \mathrm{H}) \mathrm{ppm}$.

${ }^{13}$ C NMR (151 MHz, Chloroform- $d$ ): $\delta=206.8$ (q, $\left.J=3.8 \mathrm{~Hz}\right), 133.8,133.3,132.2,130.3,129.4,129.1,129.07$, $129.03,128.8,128.7,127.2,123.3(\mathrm{q}, J=275.3 \mathrm{~Hz}), 123.0,117.5,105.0(\mathrm{q}, J=34.4 \mathrm{~Hz}) \mathrm{ppm}$.

${ }^{19}$ F NMR (565 MHz, Chloroform- $\left.d\right)$ : $\delta=-59.94$ ppm.

IR (KBr): 3061, 3032, 2925, 2856, 2324, 2077, 1946, 1901, 1767, 1594, 1488, 1448, 1400, 1370, 1296, 1167 , $1120,1071,1009,965,928,827,760,692,664 \mathrm{~cm}^{-1}$.

HRMS (ESI) $m / z:[\mathrm{M}]^{+}$mass found: 414.0227, mass calculated for $\mathrm{C}_{22} \mathrm{H}_{14} \mathrm{BrF}_{3}{ }^{+}: 414.0225$.

(4,4,4-Trifluoro-1-(4-methoxyphenyl)buta-1,2-diene-1,3-diyl)dibenzene (12j)<smiles>COc1ccc(C(=C=C(c2ccccc2)C(F)(F)F)c2ccccc2)cc1</smiles>

The titled compound was synthesized according to the general procedure GP-II, and was obtained after silica gel column chromatography using $n$-hexane : ethyl acetate $=40: 1$ as colorless oil $(93 \%, 68 \mathrm{mg})$.

${ }^{1}$ H NMR $(600 \mathrm{MHz}$, Chloroform- $d): \delta=7.38(\mathrm{~d}, J=7.8 \mathrm{~Hz}, 2 \mathrm{H}), 7.28-7.19(\mathrm{~m}, 7 \mathrm{H}), 7.17(\mathrm{~d}, J=8.7 \mathrm{~Hz}, 3 \mathrm{H})$, $6.77(\mathrm{~d}, J=8.5 \mathrm{~Hz}, 2 \mathrm{H}), 3.67(\mathrm{~s}, 3 \mathrm{H}) \mathrm{ppm}$.

${ }^{13}$ C NMR (151 MHz, Chloroform- $d$ ): $\delta=206.9$ (q, $\left.J=4.0 \mathrm{~Hz}\right), 160.2,134.6,130.0,129.0,128.9,128.8,128.7$, $128.5,127.1,126.3,123.5(\mathrm{q}, J=275.2 \mathrm{~Hz}), 118.0,114.4,104.3(\mathrm{q}, J=34.2 \mathrm{~Hz}), 55.5 \mathrm{ppm}$. Due to the overlap of aromatic carbon peaks, one carbon signal is missing.

${ }^{19}$ F NMR (565 MHz, Chloroform- $d$ ): $\delta=-59.89 \mathrm{ppm}$.

IR (KBr): 3033, 2933, 2840, 2208, 2157, 2056, 1944, 1888, 1734, 1604, 1508, 1449, 1372, 1295, 1249, 1168 , $1117,1032,966,928,833,801,764,726,695,660 \mathrm{~cm}^{-1}$.

HRMS (ESI) $m / z:[\mathrm{M}+\mathrm{H}]^{+}$mass found: 367.1293 , mass calculated for $\mathrm{C}_{23} \mathrm{H}_{18} \mathrm{~F}_{3} \mathrm{O}^{+}: 367.1304$. 


\section{4-(4,4,4-Trifluoro-1,3-diphenylbuta-1,2-dien-1-yl)-1,1'-biphenyl (12k)}<smiles>FC(F)(F)C(=Cc1ccc(-c2ccccc2)cc1)c1ccccc1</smiles>

The titled compound was synthesized according to the general procedure GP-II, and was obtained after silica gel column chromatography using $n$-hexane as colorless oil $(95 \%, 78 \mathrm{mg})$.

${ }^{1} \mathrm{H}$ NMR $(600 \mathrm{MHz}$, Chloroform- $d): \delta=7.63(\mathrm{t}, J=7.6 \mathrm{~Hz}, 4 \mathrm{H}), 7.58(\mathrm{~d}, J=7.8 \mathrm{~Hz}, 2 \mathrm{H}), 7.53-7.31(\mathrm{~m}, 13 \mathrm{H})$ ppm.

${ }^{13}$ C NMR (151 MHz, Chloroform- $d$ ): $\delta=207.1(\mathrm{q}, J=3.9 \mathrm{~Hz}), 141.7,140.6,134.3,133.2,129.8,129.2,129.07$, $129.02,128.9,128.8,128.6,127.8,127.7,127.2,127.19,127.18,123.5$ (q, $J=275.3 \mathrm{~Hz}), 118.1,104.7(\mathrm{q}, J=$ $34.2 \mathrm{~Hz}) \mathrm{ppm}$.

${ }^{19}$ F NMR $(565 \mathrm{MHz}$, Chloroform- $d$ ): $\delta=-59.85 \mathrm{ppm}$.

IR (KBr): 3060, 3032, 2925, 2856, 2236, 2156, 2020, 1945, 1803, 1752, 1679, 1599, 1489, 1448, 1407, 1373 , $1296,1166,1119,1032,1004,967,927,841,762,731,693,662 \mathrm{~cm}^{-1}$.

HRMS (ESI) $m / z:[M]^{+}$mass found: 412.1431 , mass calculated for $\mathrm{C}_{28} \mathrm{H}_{19} \mathrm{~F}_{3}{ }^{+}: 412.1433$.

\section{(4,4,4-Trifluoro-1-(m-tolyl)buta-1,2-diene-1,3-diyl)dibenzene (12l)}<smiles>Cc1cccc(C(=C=C(c2ccccc2)c2ccccc2)c2ccccc2)c1</smiles>

The titled compound was synthesized according to the general procedure GP-II, and was obtained after silica gel column chromatography using $n$-hexane as colorless oil $(87 \%, 61 \mathrm{mg})$.

${ }^{1} \mathrm{H}$ NMR $(600 \mathrm{MHz}$, Chloroform- $d$ ): $\delta=7.56(\mathrm{~d}, J=7.9 \mathrm{~Hz}, 2 \mathrm{H}), 7.44-7.37(\mathrm{~m}, 7 \mathrm{H}), 7.34(\mathrm{t}, J=7.4 \mathrm{~Hz}, 1 \mathrm{H})$, $7.31(\mathrm{t}, J=7.8 \mathrm{~Hz}, 1 \mathrm{H}), 7.25-7.19(\mathrm{~m}, 3 \mathrm{H}), 2.38(\mathrm{~s}, 3 \mathrm{H}) \mathrm{ppm}$.

${ }^{13}$ C NMR (151 MHz, Chloroform- $d$ ): $\delta=206.9$ (q, $\left.J=3.6 \mathrm{~Hz}\right), 138.7,134.4,134.2,129.8,129.6,129.3,129.0$, $128.9,128.8,128.79,128.74,128.5,127.1,126.0,123.5(\mathrm{q}, J=275.2 \mathrm{~Hz}), 118.4,104.4(\mathrm{q}, J=34.3 \mathrm{~Hz}), 21.6$ ppm.

${ }^{19}$ F NMR $(565 \mathrm{MHz}$, Chloroform- $d$ ): $\delta=-59.89 \mathrm{ppm}$.

IR (KBr): 3032, 2925, 2859, 2161, 2039, 1947, 1882, 1764, 1701, 1600, 1492, 1448, 1372, 1296, 1209, 1165 , $1119,1032,999,928,838,763,694,663 \mathrm{~cm}^{-1}$.

HRMS (ESI) $m / z:[\mathrm{M}]^{+}$mass found: 350.1275 , mass calculated for $\mathrm{C}_{23} \mathrm{H}_{17} \mathrm{~F}_{3}{ }^{+}: 350.1276$. 
<smiles>Cc1ccccc1/C(=N/C(=C\c1ccccc1)C(F)(F)F)c1ccccc1</smiles>

The titled compound was synthesized according to the general procedure GP-II, and was obtained after silica gel column chromatography using $n$-hexane as white solid $(93 \%, 65 \mathrm{mg})$.

${ }^{1} \mathrm{H}$ NMR (600 MHz, Chloroform- $d$ ): $\delta=7.58(\mathrm{~d}, J=7.8 \mathrm{~Hz}, 2 \mathrm{H}), 7.43(\mathrm{t}, J=7.7 \mathrm{~Hz}, 2 \mathrm{H}), 7.40-7.26(\mathrm{~m}, 10 \mathrm{H})$, $2.26(\mathrm{~s}, 3 \mathrm{H}) \mathrm{ppm}$.

${ }^{13} \mathbf{C}$ NMR (151 MHz, Chloroform- $d$ ): $\delta=205.5$ (q, $\left.J=4.0 \mathrm{~Hz}\right), 137.1,134.0,133.7,130.8,130.2,130.0,129.1$, $129.0,128.8,128.6,128.5,127.3,126.4,123.6$ (q, $J=275.1 \mathrm{~Hz}), 116.5,104.1$ (q, $J=34.3 \mathrm{~Hz}), 20.2 \mathrm{ppm}$. Due to the overlap of aromatic carbon peaks, one carbon signal is missing.

${ }^{19}$ F NMR $(565 \mathrm{MHz}$, Chloroform- $d$ ): $\delta=-59.68 \mathrm{ppm}$.

IR (KBr): 3062, 3022, 2924, 2856, 2319, 2076, 1944, 1891, 1808, 1736, 1598, 1491, 1451, 1375, 1296, 1123 , $1031,999,928,868,839,804,763,725,692,661 \mathrm{~cm}^{-1}$.

HRMS (ESI) $m / z:[M]^{+}$mass found: 350.1276 , mass calculated for $\mathrm{C}_{23} \mathrm{H}_{17} \mathrm{~F}_{3}{ }^{+}: 350.1276$.

(4,4,4-Trifluoro-1-(2-fluorophenyl)buta-1,2-diene-1,3-diyl)dibenzene (12n)<smiles></smiles>

The titled compound was synthesized according to the general procedure GP-II, and was obtained after silica gel column chromatography using $n$-hexane as colorless oil $(82 \%, 58 \mathrm{mg})$.

${ }^{1} \mathrm{H}$ NMR $(600 \mathrm{MHz}$, Chloroform- $d): \delta=7.57(\mathrm{~d}, J=7.9 \mathrm{~Hz}, 2 \mathrm{H}), 7.47-7.33(\mathrm{~m}, 10 \mathrm{H}), 7.21(\mathrm{t}, J=7.5 \mathrm{~Hz}, 1 \mathrm{H})$, $7.17(\mathrm{t}, J=9.1 \mathrm{~Hz}, 1 \mathrm{H}) \mathrm{ppm}$.

${ }^{13}$ C NMR (151 MHz, Chloroform- $d$ ): $\delta=206.9(\mathrm{q}, J=3.9 \mathrm{~Hz}), 160.4(\mathrm{~d}, J=250.4 \mathrm{~Hz}), 133.7,131.5(\mathrm{~d}, J=2.6$ $\mathrm{Hz}), 130.6(\mathrm{~d}, J=8.0 \mathrm{~Hz}), 129.4,129.02,129.00,128.8,128.7,127.7,127.3,124.6(\mathrm{~d}, J=3.7 \mathrm{~Hz}), 123.4(\mathrm{q}, J=$ $275.0 \mathrm{~Hz}), 122.0(\mathrm{~d}, J=14.6 \mathrm{~Hz}), 116.4(\mathrm{~d}, J=21.5 \mathrm{~Hz}), 112.2,104.4(\mathrm{q}, J=34.0 \mathrm{~Hz}) \mathrm{ppm}$.

${ }^{19}$ F NMR $(565 \mathrm{MHz}$, Chloroform- $d$ ) : $\delta=-59.95(\mathrm{~s}, 3 \mathrm{~F}),-111.58--111.62(\mathrm{~m}, 1 \mathrm{~F}) \mathrm{ppm}$.

IR (KBr): 3063, 2926, 2856, 2157, 1949, 1803, 1765, 1582, 1491, 1450, 1376, 1297, 1259, 1221, 1168, 1120 , 1032, 999, 929, 861, 815, 757, $662 \mathrm{~cm}^{-1}$.

HRMS (ESI) $m / z$ : [M] mass found: 354.1026 , mass calculated for $\mathrm{C}_{22} \mathrm{H}_{14} \mathrm{~F}_{4}{ }^{+}: 354.1026$. 
<smiles>FC(F)(F)/C(=C/c1ccccc1Cl)c1ccccc1</smiles>

The titled compound was synthesized according to the general procedure GP-II, and was obtained after silica gel column chromatography using $n$-hexane as colorless oil $(90 \%, 67 \mathrm{mg})$.

${ }^{1}$ H NMR $(600 \mathrm{MHz}$, Chloroform- $d): \delta=7.60(\mathrm{~d}, J=7.8 \mathrm{~Hz}, 2 \mathrm{H}), 7.53-7.49(\mathrm{~m}, 1 \mathrm{H}), 7.47-7.44(\mathrm{~m}, 1 \mathrm{H}), 7.43$ $-7.33(\mathrm{~m}, 8 \mathrm{H}), 7.29-7.26(\mathrm{~m}, 2 \mathrm{H}) \mathrm{ppm}$.

${ }^{13}$ C NMR (151 MHz, Chloroform- $d$ ): $\delta=206.0(\mathrm{q}, J=3.7 \mathrm{~Hz}), 134.2$, 133.3, 131.8, 130.3, 130.1, 129.3, 129.04, $129.01,128.72,128.71,127.4,127.3,127.2,123.4(\mathrm{q}, J=275.1 \mathrm{~Hz}), 115.5,105.0(\mathrm{q}, J=34.3 \mathrm{~Hz}) \mathrm{ppm}$. Due to the overlap of aromatic carbon peaks, one carbon signal is missing.

${ }^{19}$ F NMR (565 MHz, Chloroform- $d$ ): $\delta=-59.77 \mathrm{ppm}$.

IR (KBr): 3063, 2926, 2161, 2035, 1950, 1805, 1738, 1597, 1493, 1445, 1376, 1297, 1168, 1120, 1054, 1034, $929,866,838,756,728,690,662 \mathrm{~cm}^{-1}$.

HRMS (ESI) $\mathrm{m} / z$ : [M] $]^{+}$mass found: 370.0730 , mass calculated for $\mathrm{C}_{22} \mathrm{H}_{14} \mathrm{ClF}_{3}{ }^{+}: 370.0730$.

\section{2-(4,4,4-Trifluoro-1,3-diphenylbuta-1,2-dien-1-yl)naphthalene (12p)}<smiles>FC(F)(F)C(=Cc1ccc2ccccc2c1)c1ccccc1</smiles>

The titled compound was synthesized according to the general procedure GP-II, and was obtained after silica gel column chromatography using $n$-hexane as colorless oil $(93 \%, 72 \mathrm{mg})$.

${ }^{1} \mathbf{H}$ NMR $(600 \mathrm{MHz}$, Chloroform- $d): \delta=7.90-7.85(\mathrm{~m}, 2 \mathrm{H}), 7.85-7.79(\mathrm{~m}, 2 \mathrm{H}), 7.60(\mathrm{~d}, J=7.8 \mathrm{~Hz}, 2 \mathrm{H}), 7.55$ (dd, $J=8.6,1.8 \mathrm{~Hz}, 1 \mathrm{H}), 7.54-7.47(\mathrm{~m}, 4 \mathrm{H}), 7.47-7.38(\mathrm{~m}, 5 \mathrm{H}), 7.36(\mathrm{t}, J=7.3 \mathrm{~Hz}, 1 \mathrm{H}) \mathrm{ppm}$.

${ }^{13}$ C NMR $(151 \mathrm{MHz}$, Chloroform- $d$ ): $\delta=207.4(\mathrm{q}, J=3.9 \mathrm{~Hz}), 134.4,133.5,133.4,131.7,129.8,129.1,129.0$, $128.9,128.8,128.7,128.6,128.4,128.1,127.9,127.2,126.8,126.7,126.3,123.5$ (d, $J=275.2 \mathrm{~Hz}), 118.5,104.7$ $(\mathrm{q}, J=34.3 \mathrm{~Hz}) \mathrm{ppm}$.

${ }^{19}$ F NMR (565 MHz, Chloroform- $d$ ): $\delta=-59.83 \mathrm{ppm}$.

IR (KBr): 3059, 2924, 2854, 2318, 2074, 1944, 1760, 1598, 1495, 1447, 1378, 1296, 1237, 1167, 1120, 1030, $1000,963,928,859,816,748,694 \mathrm{~cm}^{-1}$.

HRMS (ESI) $\mathrm{m} / \mathrm{z}$ : [M] $]^{+}$mass found: 386.1276 , mass calculated for $\mathrm{C}_{26} \mathrm{H}_{17} \mathrm{~F}_{3}{ }^{+}: 386.1276$. 
<smiles>FC(F)(F)C(=NC(c1ccccc1)c1ccccc1)c1cccc2ccccc12</smiles>

The titled compound was synthesized according to the general procedure GP-II, and was obtained after silica gel column chromatography using $n$-hexane as white solid $(92 \%, 71 \mathrm{mg})$.

${ }^{1}$ H NMR $(600 \mathrm{MHz}$, Chloroform- $d$ ): $\delta=7.95-7.87(\mathrm{~m}, 3 \mathrm{H}), 7.60(\mathrm{~d}, J=7.0 \mathrm{~Hz}, 1 \mathrm{H}), 7.58-7.54(\mathrm{~m}, 3 \mathrm{H}), 7.50$ (t, $J=7.5 \mathrm{~Hz}, 1 \mathrm{H}), 7.44-7.35(\mathrm{~m}, 3 \mathrm{H}), 7.35-7.25(\mathrm{~m}, 6 \mathrm{H}) \mathrm{ppm}$.

${ }^{13}$ C NMR (151 MHz, Chloroform- $d$ ): $\delta=206.2$ (q, $\left.J=3.6 \mathrm{~Hz}\right), 134.4,134.0,131.9,131.7,129.8,129.3,129.04$, $129.02,128.65,128.60,128.5,127.9,127.4,127.3,126.6,126.3,126.1,125.7,123.5$ (q, $J=275.2 \mathrm{~Hz}), 115.7$, $104.2(\mathrm{q}, J=34.2 \mathrm{~Hz}) \mathrm{ppm}$.

${ }^{19}$ F NMR $(565 \mathrm{MHz}$, Chloroform- $d$ ): $\delta=-59.72 \mathrm{ppm}$.

IR (KBr): 3347, 3058, 2923, 2854, 2667, 2318, 2079, 1937, 1815, 1732, 1592, 1493, 1446, 1394, 1369, 1338, $1292,1252,1206,1163,1123,1028,970,926,863,797,770,694 \mathrm{~cm}^{-1}$.

HRMS (ESI) $m / z$ : [M] mass found: 386.1275 , mass calculated for $\mathrm{C}_{26} \mathrm{H}_{17} \mathrm{~F}_{3}{ }^{+}: 386.1276$.

\section{2-(4,4,4-Trifluoro-1,3-diphenylbuta-1,2-dien-1-yl)thiophene (12r)}<smiles>FC(F)(F)C(=C=C(c1ccccc1)c1cccs1)c1ccccc1</smiles>

The titled compound was synthesized according to the general procedure GP-II, and was obtained after silica gel column chromatography using $n$-hexane as white solid $(76 \%, 52 \mathrm{mg})$.

${ }^{1}$ H NMR (600 MHz, Chloroform- $d$ ): $\delta=7.60-7.50(\mathrm{~m}, 4 \mathrm{H}), 7.46-7.31(\mathrm{~m}, 7 \mathrm{H}), 7.14-7.04(\mathrm{~m}, 2 \mathrm{H}) \mathrm{ppm}$.

${ }^{13}$ C NMR (151 MHz, Chloroform- $d$ ) : $\delta=206.9$ (q, $\left.J=3.9 \mathrm{~Hz}\right), 136.7,134.1,129.6,129.1,129.0,128.9,128.7$, $128.6,127.94,127.92,127.4,127.0,123.1(\mathrm{q}, J=275.5 \mathrm{~Hz}), 113.1,104.7(\mathrm{q}, J=34.5 \mathrm{~Hz}) \mathrm{ppm}$.

${ }^{19}$ F NMR (565 MHz, Chloroform- $d$ ): $\delta=-60.12 \mathrm{ppm}$.

IR (KBr): 3064, 3032, 2926, 2674, 2325, 2089, 1884, 1803, 1702, 1598, 1530, 1493, 1446, 1379, 1295, 1260 , $1228,1166,1121,1033,998,927,834,761,694,663 \mathrm{~cm}^{-1}$.

HRMS (ESI) $\mathrm{m} / z$ : $[\mathrm{M}]^{+}$mass found: 342.0680 , mass calculated for $\mathrm{C}_{20} \mathrm{H}_{13} \mathrm{~F}_{3} \mathrm{~S}^{+}: 342.0684$.

\section{5-(3,3,3-Trifluoro-2-phenylprop-1-en-1-ylidene)-10,11-dihydro-5H-dibenzo[ $[a, d][7]$ annulene (12s)}<smiles>FC(F)(F)C(=C=C1c2ccccc2CCc2ccccc21)c1ccccc1</smiles>

The titled compound was synthesized according to the general procedure GP-II, and was obtained after silica gel column chromatography using $n$-hexane as colorless oil $(86 \%, 62 \mathrm{mg})$.

${ }^{1}$ H NMR $(600 \mathrm{MHz}$, Chloroform- $d$ ): $\delta=7.49(\mathrm{~d}, J=7.8 \mathrm{~Hz}, 2 \mathrm{H}), 7.43(\mathrm{~d}, J=7.4 \mathrm{~Hz}, 2 \mathrm{H}), 7.37(\mathrm{t}, J=7.6 \mathrm{~Hz}$, $2 \mathrm{H}), 7.30(\mathrm{t}, J=7.2 \mathrm{~Hz}, 1 \mathrm{H}), 7.26(\mathrm{t}, J=7.1 \mathrm{~Hz}, 2 \mathrm{H}), 7.21-7,19(\mathrm{~m}, 4 \mathrm{H}), 3.35-3.30(\mathrm{~m}, 2 \mathrm{H}), 3.17-3.11(\mathrm{~m}$, 2H) $\mathrm{ppm}$.

${ }^{13}$ C NMR $(151 \mathrm{MHz}$, Chloroform- $d): \delta=207.5(\mathrm{q}, J=4.1 \mathrm{~Hz}), 139.5,133.9,130.0,129.8,129.2,128.9,128.8$, $128.4,127.2,126.7,123.8(\mathrm{q}, J=275.1 \mathrm{~Hz}), 119.1,102.6(\mathrm{q}, J=34.0 \mathrm{~Hz}), 33.5 \mathrm{ppm}$.

${ }^{19}$ F NMR $(565 \mathrm{MHz}$, Chloroform- $d$ ): $\delta=-59.87 \mathrm{ppm}$.

IR (KBr): 3069, 3022, 2922, 2855, 2325, 2086, 1945, 1807, 1732, 1599, 1488, 1447, 1369, 1300, 1185, 1159, $1116,1035,928,892,836,806,752,717,691,663 \mathrm{~cm}^{-1}$.

HRMS (ESI) $m / z$ : [M] mass found: 362.1277 , mass calculated for $\mathrm{C}_{24} \mathrm{H}_{17} \mathrm{~F}_{3}{ }^{+}: 362.1276$. 
<smiles>FC(F)(F)C(=C=C1c2ccccc2C=Cc2ccccc21)c1ccccc1</smiles>

The titled compound was synthesized according to the general procedure GP-II, and was obtained after silica gel column chromatography using $n$-hexane as white solid $(81 \%, 58 \mathrm{mg})$.

${ }^{1}$ H NMR $(600 \mathrm{MHz}$, Chloroform- $d): \delta=7.49(\mathrm{~d}, J=7.0 \mathrm{~Hz}, 2 \mathrm{H}), 7.42(\mathrm{~d}, J=7.5 \mathrm{~Hz}, 2 \mathrm{H}), 7.40-7.31(\mathrm{~m}, 8 \mathrm{H})$, $7.28(\mathrm{~d}, J=7.2 \mathrm{~Hz}, 1 \mathrm{H}), 6.89(\mathrm{~s}, 2 \mathrm{H}) \mathrm{ppm}$.

${ }^{13}$ C NMR (151 MHz, Chloroform- $d$ ): $\delta=207.3(\mathrm{q}, J=4.2 \mathrm{~Hz}), 134.8,133.6,131.2,129.7,129.5,129.3,128.86$, $128.85,128.6,128.3,127.2,124.0(\mathrm{q}, J=275.0 \mathrm{~Hz}), 118.5,102.1(\mathrm{q}, J=33.9 \mathrm{~Hz}) \mathrm{ppm}$.

${ }^{19}$ F NMR (565 MHz, Chloroform- $\left.d\right): \delta=-59.89$ ppm.

IR (KBr): 3063, 3026, 2926, 2859, 2162, 2037, 1949, 1720, 1597, 1490, 1435, 1374, 1297, 1227, 1163, 1121 , $1039,973,929,878,849,798,765,742,691,665 \mathrm{~cm}^{-1}$.

HRMS (ESI) $m / z:[\mathrm{M}]^{+}$mass found: 360.1122 , mass calculated for $\mathrm{C}_{24} \mathrm{H}_{15} \mathrm{~F}_{3}{ }^{+}: 360.1120$.

\section{(3-(4-Chlorophenyl)-4,4,4-trifluorobuta-1,2-diene-1,1-diyl)dibenzene (12u)}<smiles>FC(F)(F)C(=C=C(c1ccccc1)c1ccccc1)c1ccc(Cl)cc1</smiles>

The titled compound was synthesized according to the general procedure GP-II, and was obtained after silica gel column chromatography using $n$-hexane as colorless oil $(92 \%, 68 \mathrm{mg})$.

${ }^{1}$ H NMR (600 MHz, Chloroform- $d$ ): $\delta=7.46(\mathrm{~d}, J=8.2 \mathrm{~Hz}, 2 \mathrm{H}), 7.43-7.34(\mathrm{~m}, 12 \mathrm{H}) \mathrm{ppm}$.

${ }^{13}$ C NMR (151 MHz, Chloroform- $d$ ): $\delta=206.9$ (q, $\left.J=3.8 \mathrm{~Hz}\right), 134.6,134.0,129.2,129.0,128.9,128.7,128.4$, $128.3,123.2(\mathrm{q}, J=275.1 \mathrm{~Hz}), 118.7,103.7(\mathrm{q}, J=34.7 \mathrm{~Hz}) \mathrm{ppm}$.

${ }^{19}$ F NMR $(565 \mathrm{MHz}$, Chloroform- $d)$ : $\delta=-60.10 \mathrm{ppm}$.

IR (KBr): 3061, 2926, 2857, 2185, 1948, 1897, 1765, 1710, 1596, 1492, 1450, 1369, 1296, 1170, 1120, 1015 , 966, 927, 902, 828, 766, $695 \mathrm{~cm}^{-1}$.

HRMS (ESI) $m / z:[\mathrm{M}]^{+}$mass found: 370.0725 , mass calculated for $\mathrm{C}_{22} \mathrm{H}_{14} \mathrm{ClF}_{3}{ }^{+}: 370.0730$.

\section{(4,4,4-Trifluoro-3-(p-tolyl)buta-1,2-diene-1,1-diyl)dibenzene (12v)}<smiles>Cc1ccc(C(=C=C(c2ccccc2)c2ccccc2)C(F)(F)F)cc1</smiles>

The titled compound was synthesized according to the general procedure GP-II, and was obtained after silica gel column chromatography using $n$-hexane as colorless oil $(89 \%, 62 \mathrm{mg})$.

${ }^{1}$ H NMR $(600 \mathrm{MHz}$, Chloroform- $d): \delta=7.43(\mathrm{~d}, J=8.0 \mathrm{~Hz}, 2 \mathrm{H}), 7.41-7.36(\mathrm{~m}, 10 \mathrm{H}), 7.20(\mathrm{~d}, J=8.1 \mathrm{~Hz}, 2 \mathrm{H})$, 2.37 (s, 3H) ppm.

${ }^{13}$ C NMR (151 MHz, Chloroform- $d$ ): $\delta=206.7$ (q, $\left.J=4.0 \mathrm{~Hz}\right), 138.6,134.4,129.7,128.9,128.8,128.7,127.0$, $126.7,123.5$ (q, $J=275.3 \mathrm{~Hz}), 118.1,104.5$ (q, $J=34.3 \mathrm{~Hz}), 21.3 \mathrm{ppm}$.

${ }^{19}$ F NMR (565 MHz, Chloroform- $d$ ): $\delta=-60.02 \mathrm{ppm}$.

IR (KBr): 3335, 3059, 3031, 2923, 2855, 2221, 2038, 1948, 1901, 1805, 1736, 1656, 1599, 1513, 1492, 1449 , $1373,1296,1166,1118,1029,927,818,767,743,694 \mathrm{~cm}^{-1}$.

HRMS (ESI) $m / z$ : [M] $]^{+}$mass found: 350.1276 , mass calculated for $\mathrm{C}_{23} \mathrm{H}_{17} \mathrm{~F}_{3}{ }^{+}: 350.1276$. 
<smiles>Fc1ccc(C(=C=C(c2ccccc2)c2ccccc2)C(F)(F)F)cc1</smiles>

The titled compound was synthesized according to the general procedure GP-II, and was obtained after silica gel column chromatography using $n$-hexane as white solid $(93 \%, 66 \mathrm{mg})$.

${ }^{1}$ H NMR $(600 \mathrm{MHz}$, Chloroform- $d): \delta=7.51(\mathrm{dd}, J=8.0,5.5 \mathrm{~Hz}, 2 \mathrm{H}), 7.43-7.38(\mathrm{~m}, 10 \mathrm{H}), 7.09(\mathrm{t}, J=8.7 \mathrm{~Hz}$, 2H) ppm.

${ }^{13}$ C NMR (151 MHz, Chloroform- $\left.d\right): \delta=206.7(\mathrm{q}, J=3.7 \mathrm{~Hz}), 162.8(\mathrm{~d}, J=248.9 \mathrm{~Hz}), 134.2,129.0(\mathrm{~d}, J=7.5$ $\mathrm{Hz}), 128.99,128.90,128.7,125.8(\mathrm{~d}, J=3.6 \mathrm{~Hz}), 118.5,116.1(\mathrm{~d}, J=21.8 \mathrm{~Hz}), 103.7$ (q, $J=34.7 \mathrm{~Hz}) \mathrm{ppm}$. Due to the overlap of aromatic carbon peaks, one carbon signal is missing.

${ }^{19}$ F NMR $(565 \mathrm{MHz}$, Chloroform- $d$ ): $\delta=-60.23$ (s, 3F), -112.80--112.85 (m, 1F) ppm.

IR (KBr): 3065, 2925, 2856, 2325, 1892, 1764, 1734, 1649, 1601, 1508, 1449, 1368, 1292, 1236, 1171, 1123 , $1025,998,967,927,835,770,744,694,663 \mathrm{~cm}^{-1}$.

HRMS (ESI) $m / z:[\mathrm{M}]^{+}$mass found: 354.1028 , mass calculated for $\mathrm{C}_{22} \mathrm{H}_{14} \mathrm{~F}_{4}{ }^{+}: 354.1026$.

6-Methyl-1-phenyl-3-(p-tolyl)-1-(trifluoromethyl)-1H-indene (16)<smiles></smiles>

The titled compound was synthesized according to the general procedure GP-III, and was obtained after silica gel column chromatography using $n$-hexane as colorless oil $(97 \%, 71 \mathrm{mg})$.

${ }^{1}$ H NMR $(400 \mathrm{MHz}$, Chloroform- $d): \delta=7.57(\mathrm{~d}, J=8.1 \mathrm{~Hz}, 2 \mathrm{H}), 7.54(\mathrm{~d}, J=8.1 \mathrm{~Hz}, 2 \mathrm{H}), 7.47(\mathrm{~d}, J=7.8 \mathrm{~Hz}$, 2H), $7.38-7.31(\mathrm{~m}, 3 \mathrm{H}), 7.29(\mathrm{~d}, J=7.8 \mathrm{~Hz}, 2 \mathrm{H}), 7.24(\mathrm{~d}, J=7.9 \mathrm{~Hz}, 1 \mathrm{H}), 6.58(\mathrm{~s}, 1 \mathrm{H}), 2.45$ (s, 3H), 2.43 (s, 3H) $\mathrm{ppm}$.

${ }^{13}$ C NMR (101 MHz, Chloroform- $d$ ): $\delta=147.1,144.3,141.0,138.5,136.6,135.0,131.6,130.7,129.5,129.3$, $128.7,128.0,127.9,127.7,126.8$ (q, $J=282.6 \mathrm{~Hz}), 126.6,121.4,64.0$ (q, $J=26.7 \mathrm{~Hz}), 21.7,21.4 \mathrm{ppm}$.

${ }^{19}$ F NMR (376 MHz, Chloroform- $d$ ): $\delta=-67.38$ ppm.

IR (KBr): 3030, 2923, 2865, 1902, 1803, 1607, 1505, 1449, 1380, 1346, 1246, 1151, 1068, 1037, 975, 939, $889,809,758,718,695,663 \mathrm{~cm}^{-1}$.

HRMS (ESI) $m / z:[\mathrm{M}]^{+}$mass found: 364.1434 , mass calculated for $\mathrm{C}_{24} \mathrm{H}_{19} \mathrm{~F}_{3}{ }^{+}: 364.1433$. 
<smiles>COc1ccc(C2(c3ccc(C)cc3)C=C(C(F)(F)F)c3ccccc32)cc1</smiles>

The titled compound was synthesized according to the general procedure GP-IV, and was obtained after silica gel column chromatography using $n$-hexane as colorless oil $(19 \%, 14 \mathrm{mg})$.

${ }^{1}$ H NMR $(600 \mathrm{MHz}$, Chloroform- $d): \delta=7.49(\mathrm{~d}, J=7.5 \mathrm{~Hz}, 1 \mathrm{H}), 7.39-7.31(\mathrm{~m}, 2 \mathrm{H}), 7.28(\mathrm{t}, J=7.4 \mathrm{~Hz}, 1 \mathrm{H})$, $7.18(\mathrm{~s}, 1 \mathrm{H}), 7.07(\mathrm{~s}, 8 \mathrm{H}), 2.31(\mathrm{~s}, 6 \mathrm{H}) \mathrm{ppm}$.

${ }^{13}$ C NMR (151 MHz, Chloroform- $d$ ): $\delta=150.6,146.2(\mathrm{q}, J=5.0 \mathrm{~Hz}), 138.7,137.2,137.1,132.2(\mathrm{q}, J=34.3 \mathrm{~Hz})$, 129.4, 127.7, 127.6, 127.1, 125.7, 124.2 (q, $J=270.1 \mathrm{~Hz}), 121.6,65.6,21.1 \mathrm{ppm}$.

${ }^{19}$ F NMR (565 MHz, Chloroform- $d$ ): $\delta=-63.91 \mathrm{ppm}$.

IR (KBr): 3025, 2924, 2866, 2164, 2027, 1907, 1736, 1628, 1580, 1508, 1461, 1377, 1312, 1288, 1267, 1211, $1169,1121,1023,979,919,851,813,767,743,663 \mathrm{~cm}^{-1}$.

HRMS (APCI) $m / z:[\mathrm{M}+\mathrm{H}]^{+}$mass found: 365.1511, mass calculated for $\mathrm{C}_{24} \mathrm{H}_{20} \mathrm{~F}_{3}{ }^{+}: 365.1508$.

\section{References}

1. Zhang, G.; Bai, R.-X.; Li, C.-H.; Feng, C.-G.; Lin, G.-Q. Halogenation of 1,1-Diarylethylenes by NHalosuccinimides. Tetrahedron 2019, 75, 1658-1662.

2. Chang, M.-Y.; Tai, H.-Y.; Tsai, C.-Y.; Chuang, Y.-J.; Lin, Y.-T. Synthesis of substituted aryl enol ethers. Tetrahedron Lett. 2014, 55, 6482-6485.

3. Kong, W.; Che, C.; Wu, J.; Ma, L.; Zhu, G. Pd-Catalyzed Regio- and Stereoselective Addition of Boronic Acids to Silylacetylenes: A Stereodivergent Assembly of $\beta, \beta$-Disubstituted Alkenylsilanes and Alkenyl Halides. J. Org. Chem. 2014, 79, 5799-5805. 


\section{Spectra of compounds}

1-Bromo-4-(2-bromo-1-phenylvinyl)benzene (9i)

${ }^{1}$ H NMR (600 MHz, Chloroform- $d$ )

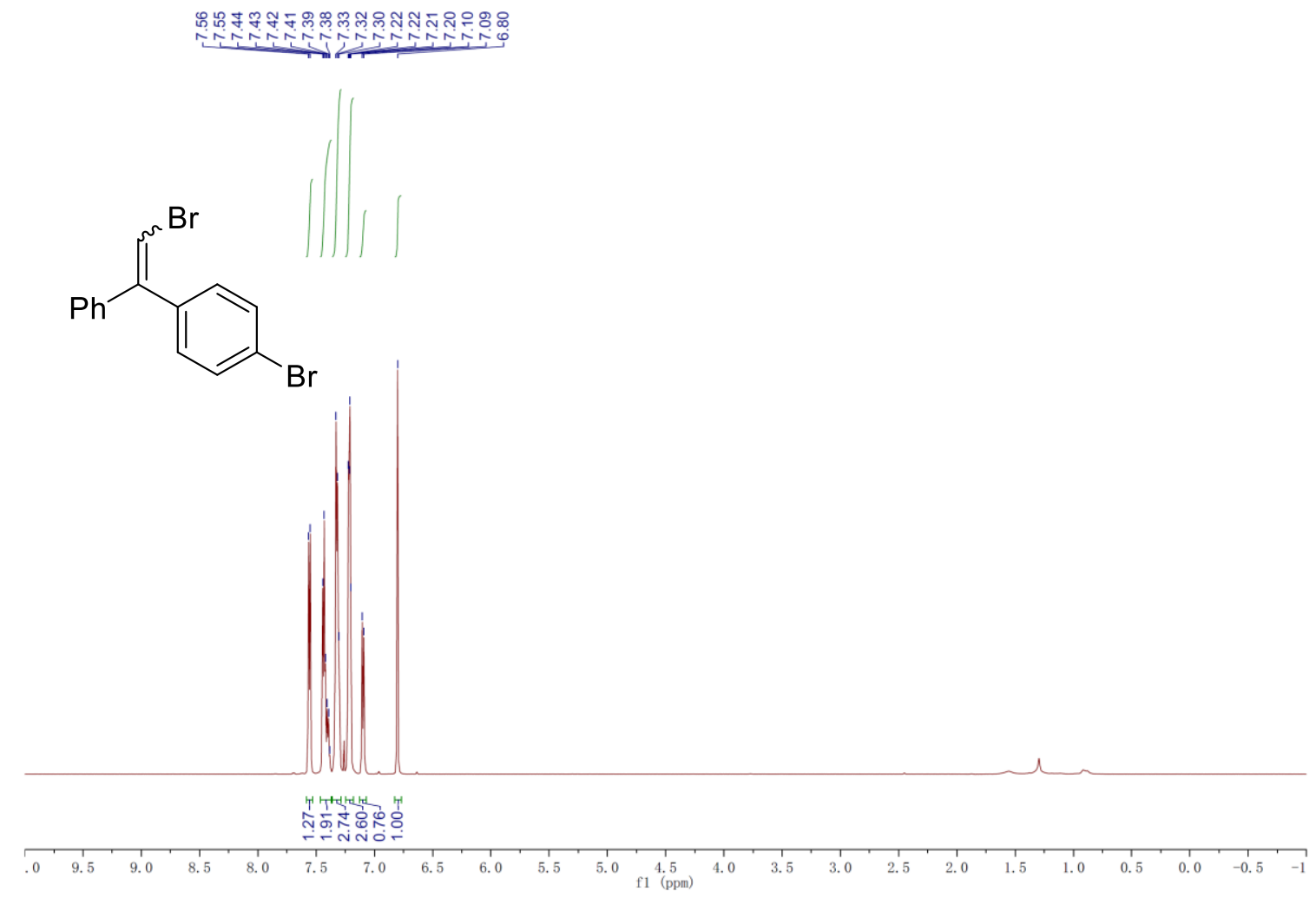

${ }^{13}$ C NMR (151 MHz, Chloroform-d)

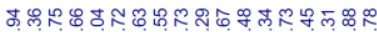

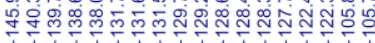<smiles>Br/C=C(\c1ccccc1)c1ccc(Br)cc1</smiles>

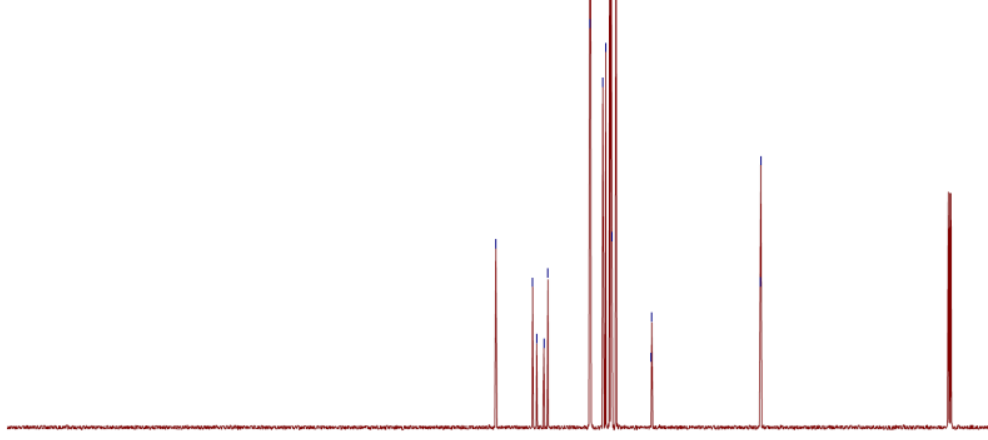


1-(2-Bromo-1-phenylvinyl)-3-methylbenzene (9l)

${ }^{1}$ H NMR (600 MHz, Chloroform-d)

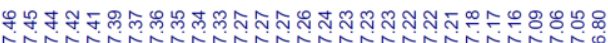

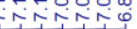

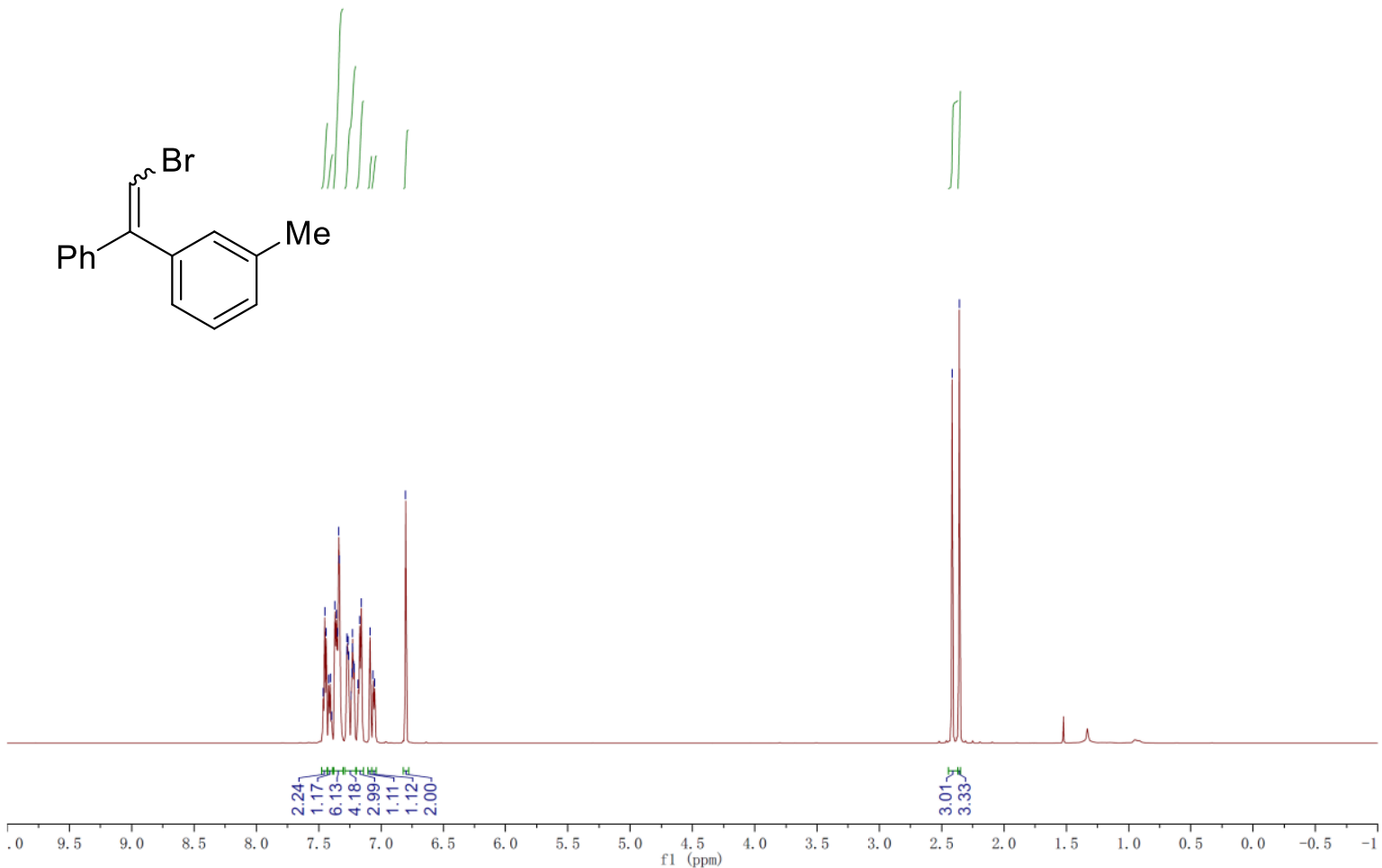

${ }^{13}$ C NMR (151 MHz, Chloroform-d)

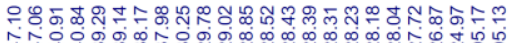

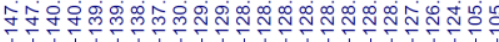

กิ่

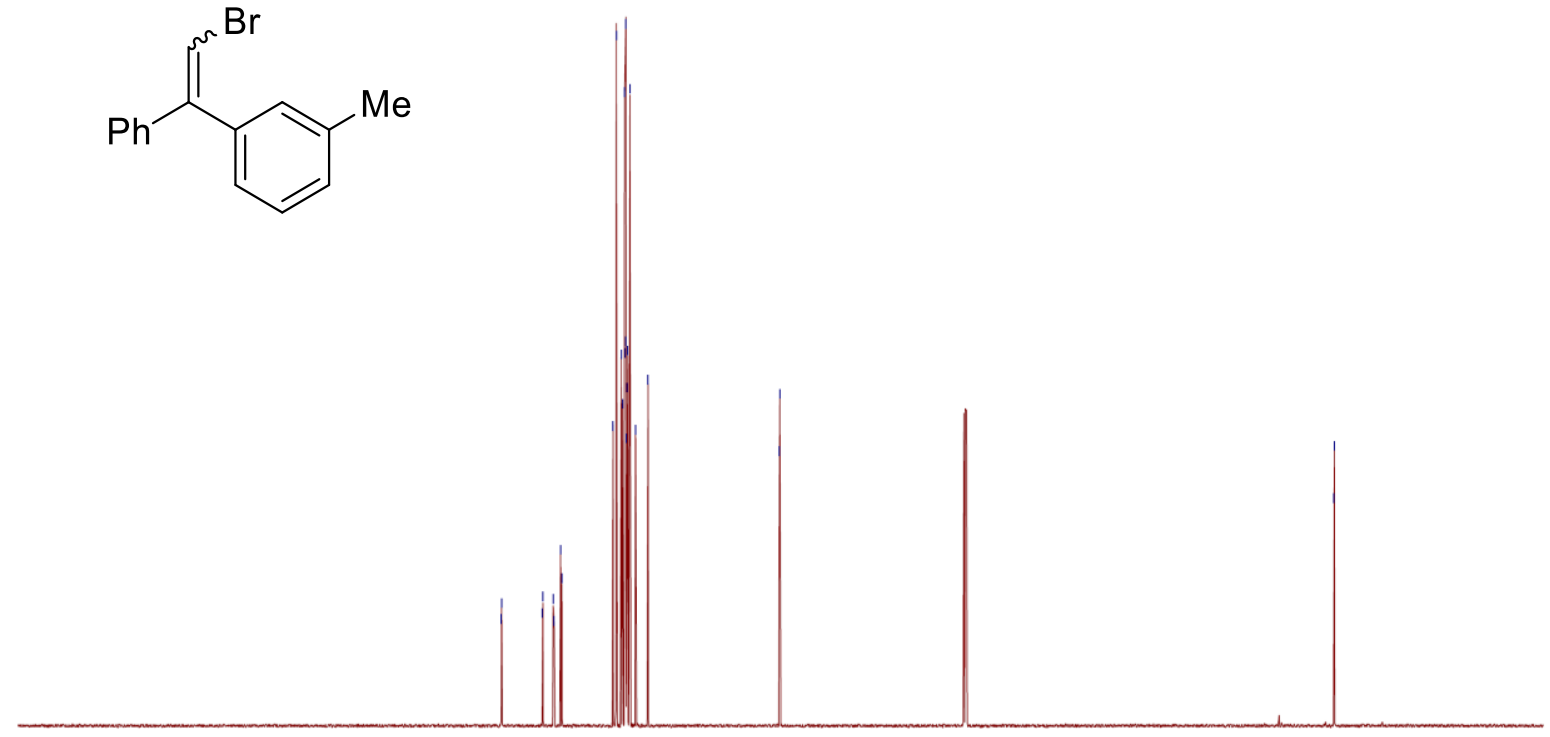

$\begin{array}{lllllllllllll}20 & 210 & 200 & 190 & 180 & 170 & 160 & 150 & 140 & 130 & 120 & 110 & 100 \\ \mathrm{f} 1 & (\mathrm{pmpm})\end{array}$ 
1-(2-Bromo-1-phenylvinyl)-2-chlorobenzene (9o)

${ }^{1}$ H NMR (600 MHz, Chloroform- $d$ )

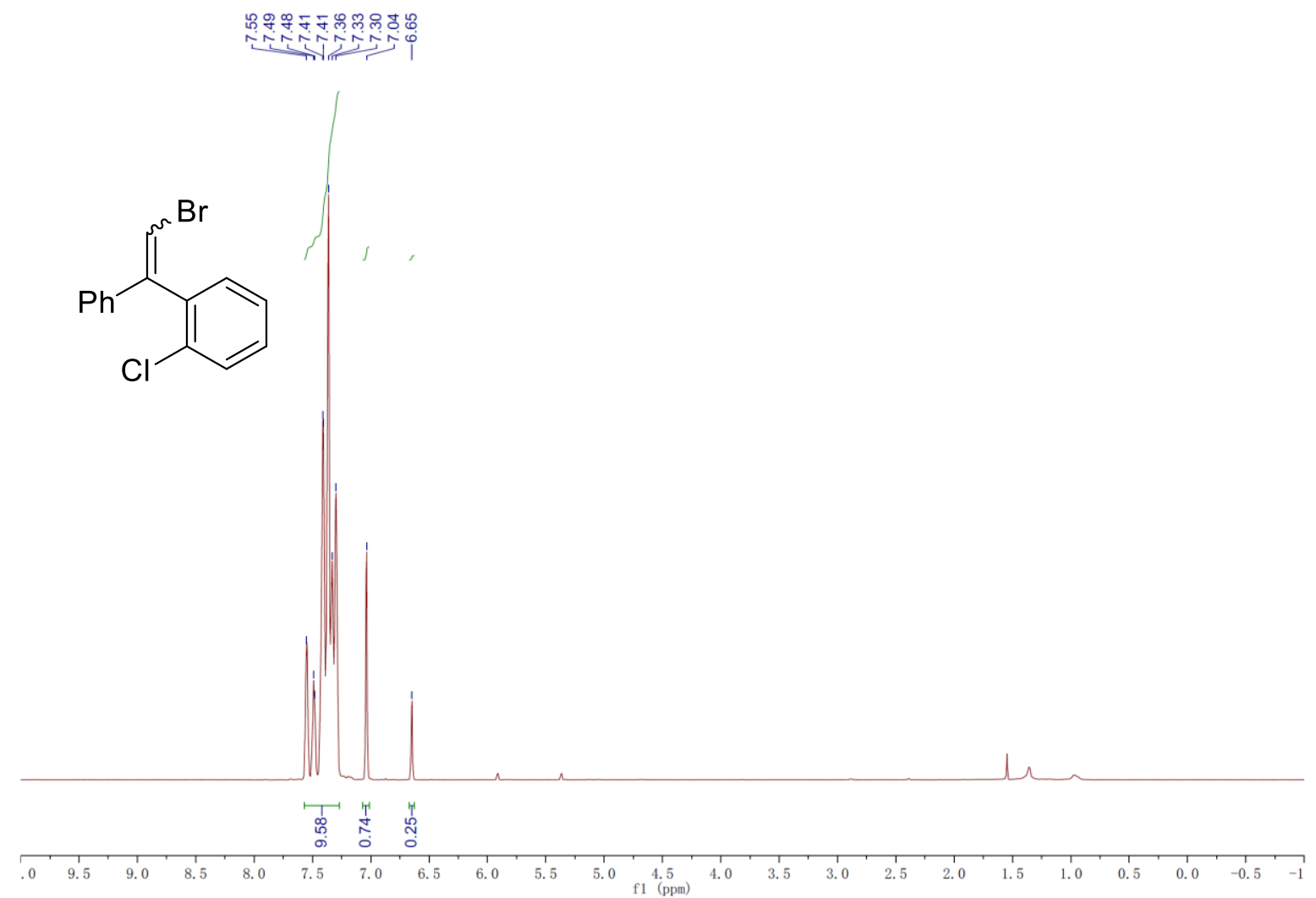

${ }^{13}$ C NMR (151 MHz, Chloroform-d)

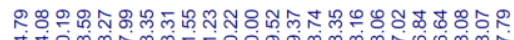

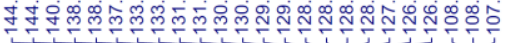

(r)

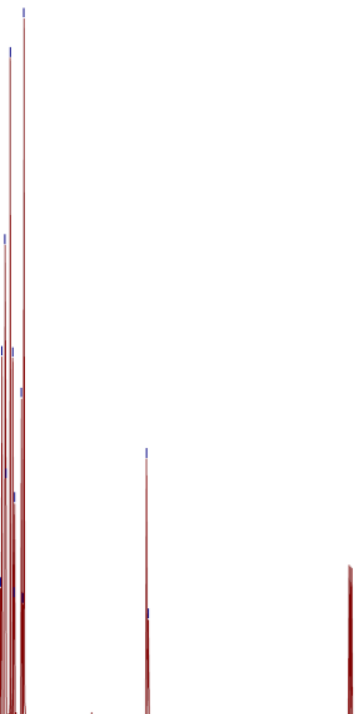

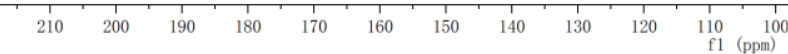


1-(2-Bromo-1-phenylvinyl)naphthalene (9q)

${ }^{1}$ H NMR (600 MHz, Chloroform- $d$ )

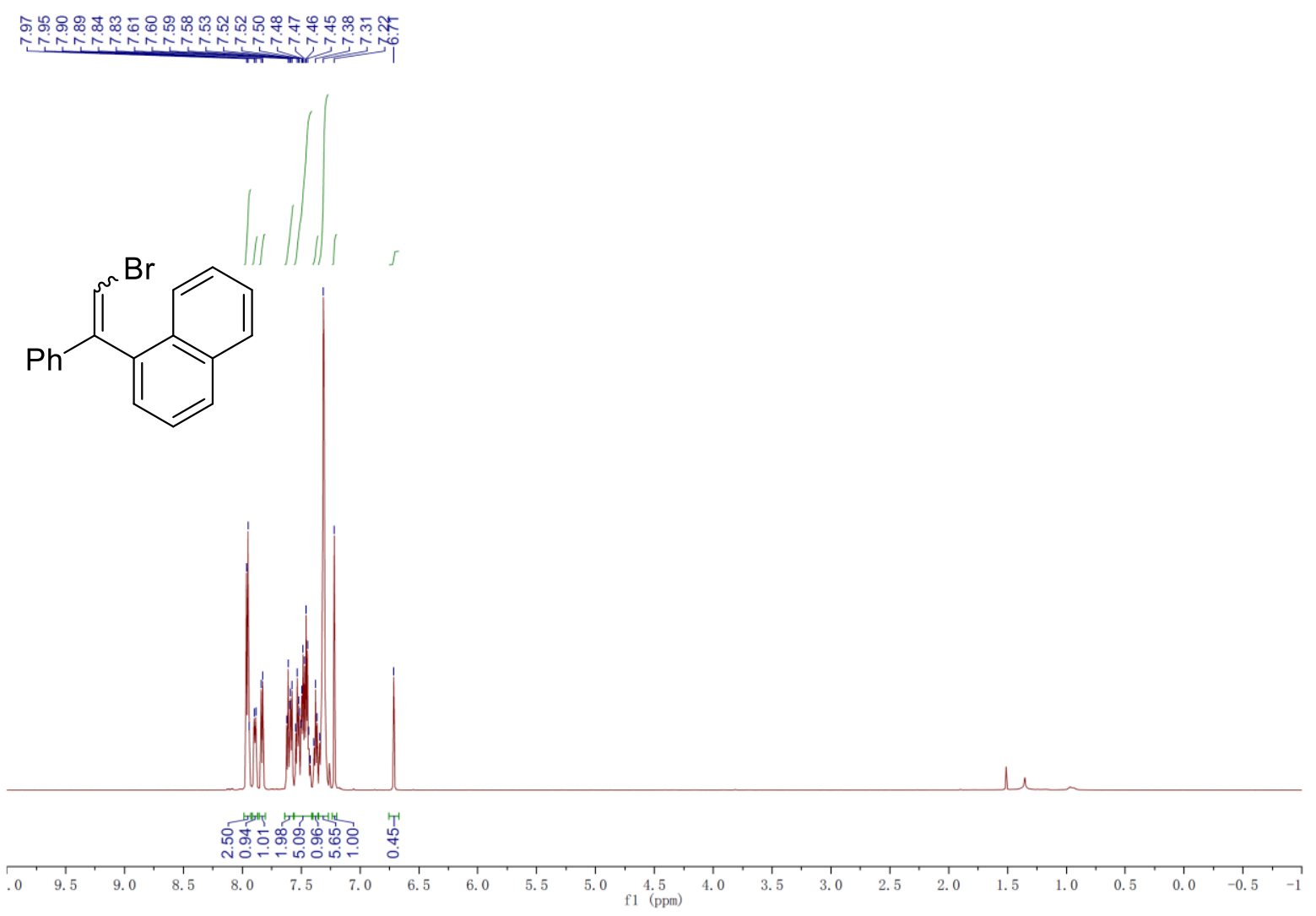

${ }^{13}$ C NMR (151 MHz, Chloroform- $d$ )

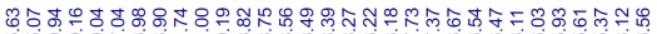

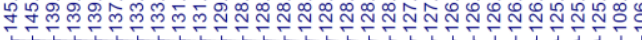

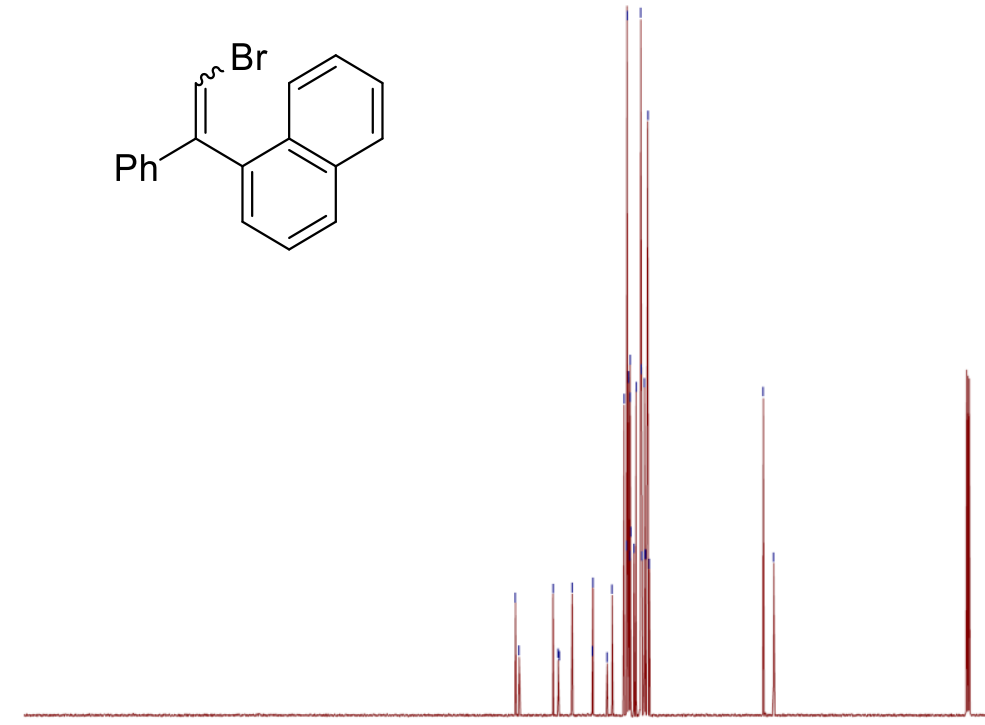

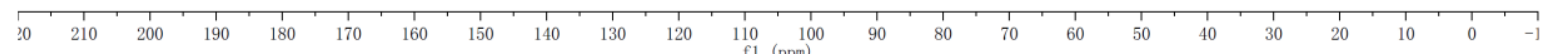


5-(Bromomethylene)-10,11-dihydro-5H-dibenzo[ $a, d][7]$ annulene $(9 \mathrm{~s})$

${ }^{1}$ H NMR (400 MHz, Chloroform- $d$ )

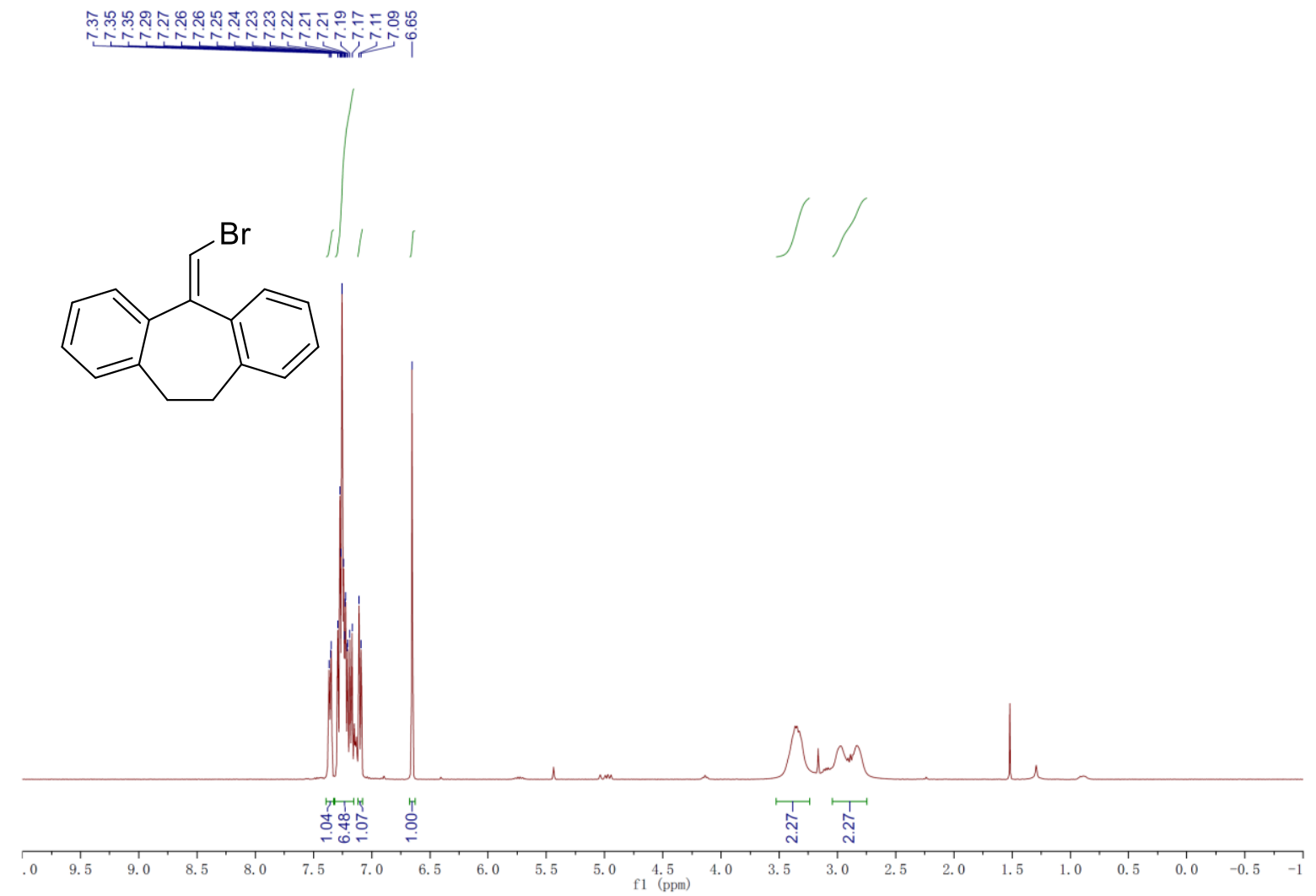

${ }^{13}$ C NMR (101 MHz, Chloroform-d)

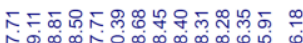

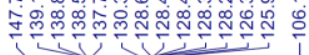

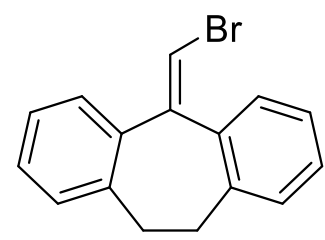

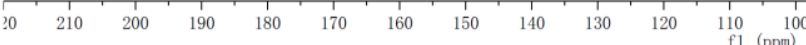


5-(Bromomethylene)-5H-dibenzo[ $[a, d][7]$ annulene (9t)

${ }^{1}$ H NMR (300 MHz, Chloroform- $d$ )

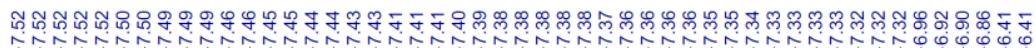

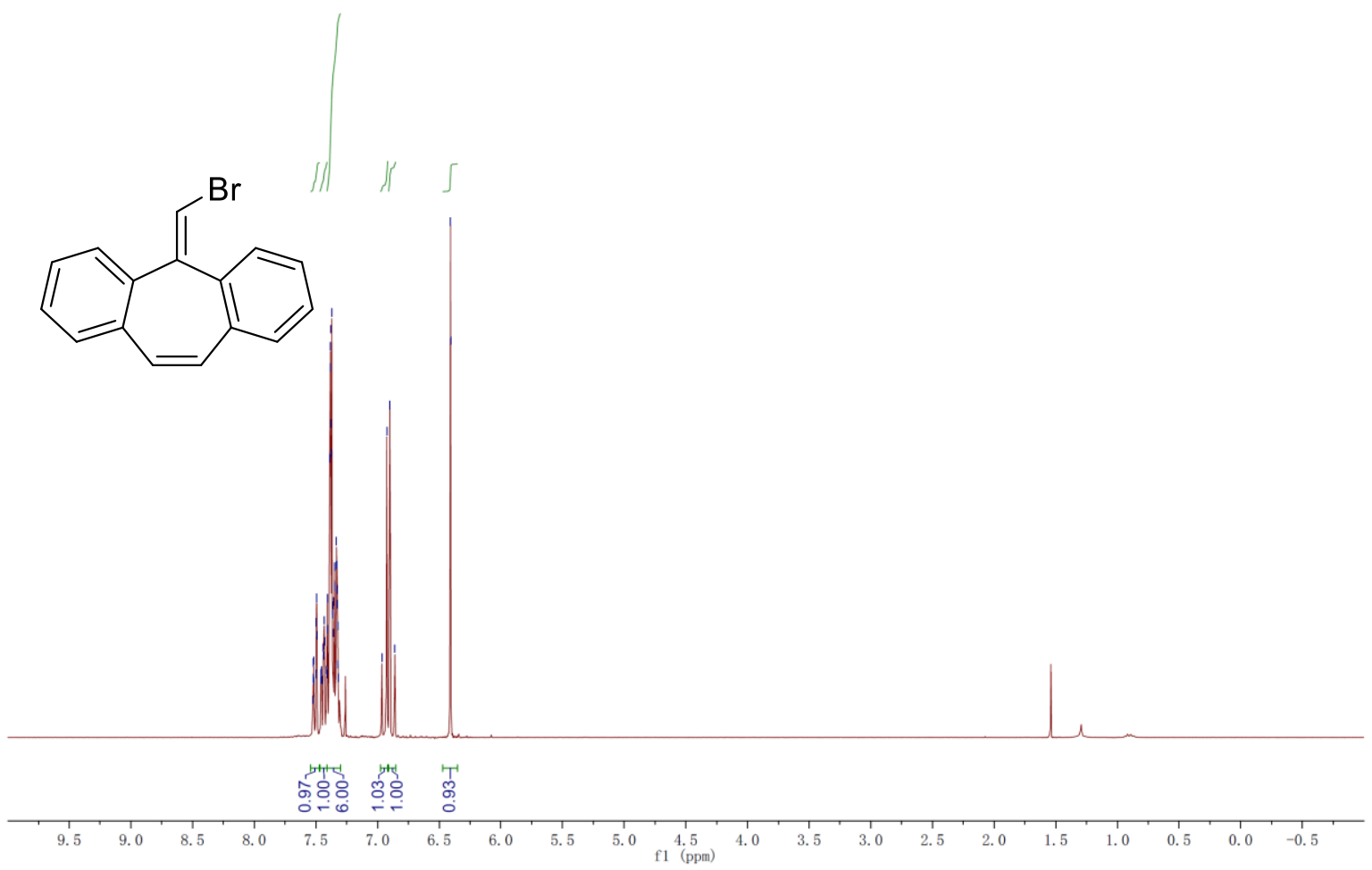

${ }^{13}$ C NMR (75 MHz, Chloroform- $d$ )

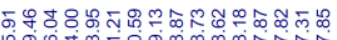

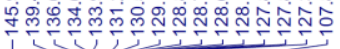<smiles>BrC=C1c2ccccc2C=Cc2ccccc21</smiles>

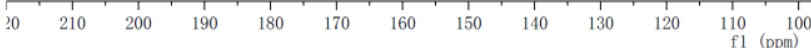


(4,4,4-Trifluorobuta-1,2-diene-1,1,3-triyl)tribenzene (12a)

${ }^{1}$ H NMR (600 MHz, Chloroform- $d$ )

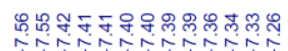<smiles>FC(F)(F)C(=C=C(c1ccccc1)c1ccccc1)c1ccccc1</smiles>

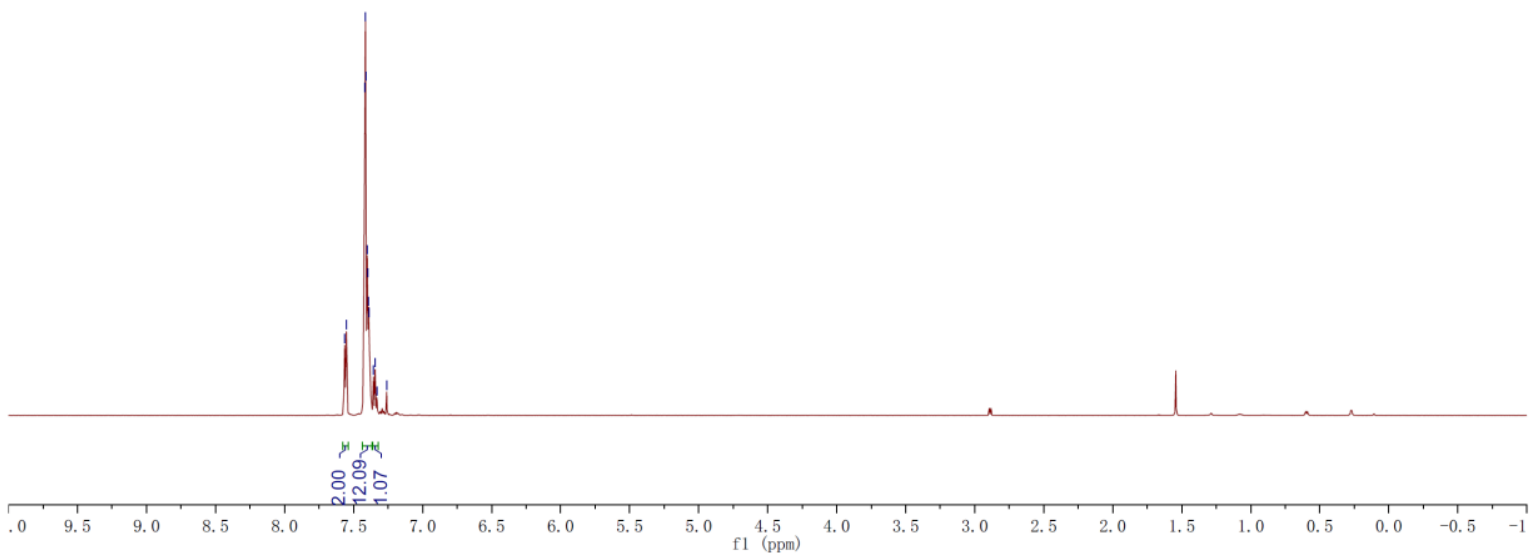

${ }^{13}$ C NMR (151 MHz, Chloroform- $d$ )

ร.850.

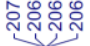

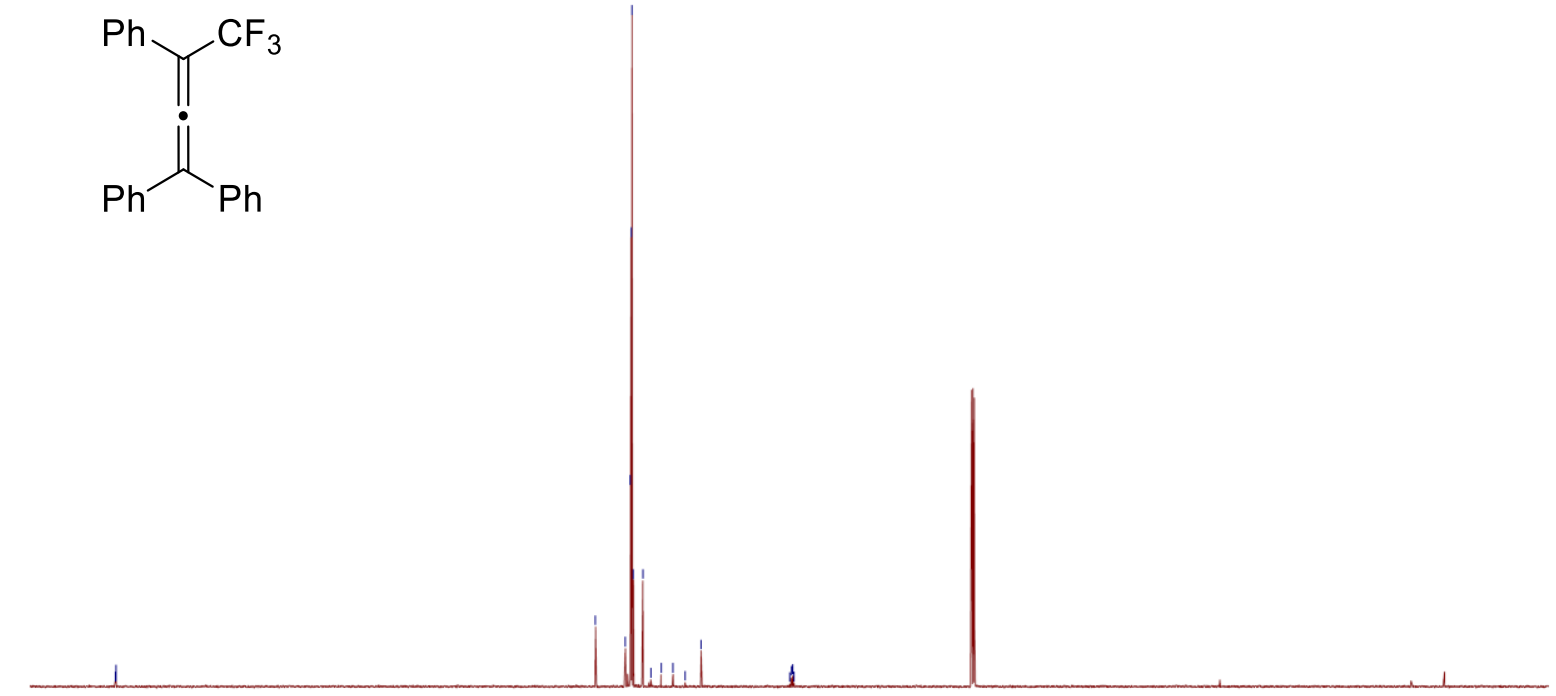

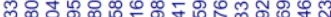

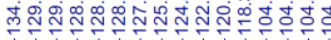

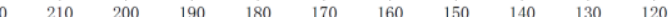

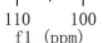

90

7 
${ }^{19}$ F NMR (565 MHz, Chloroform- $d$ )

๙্ণ

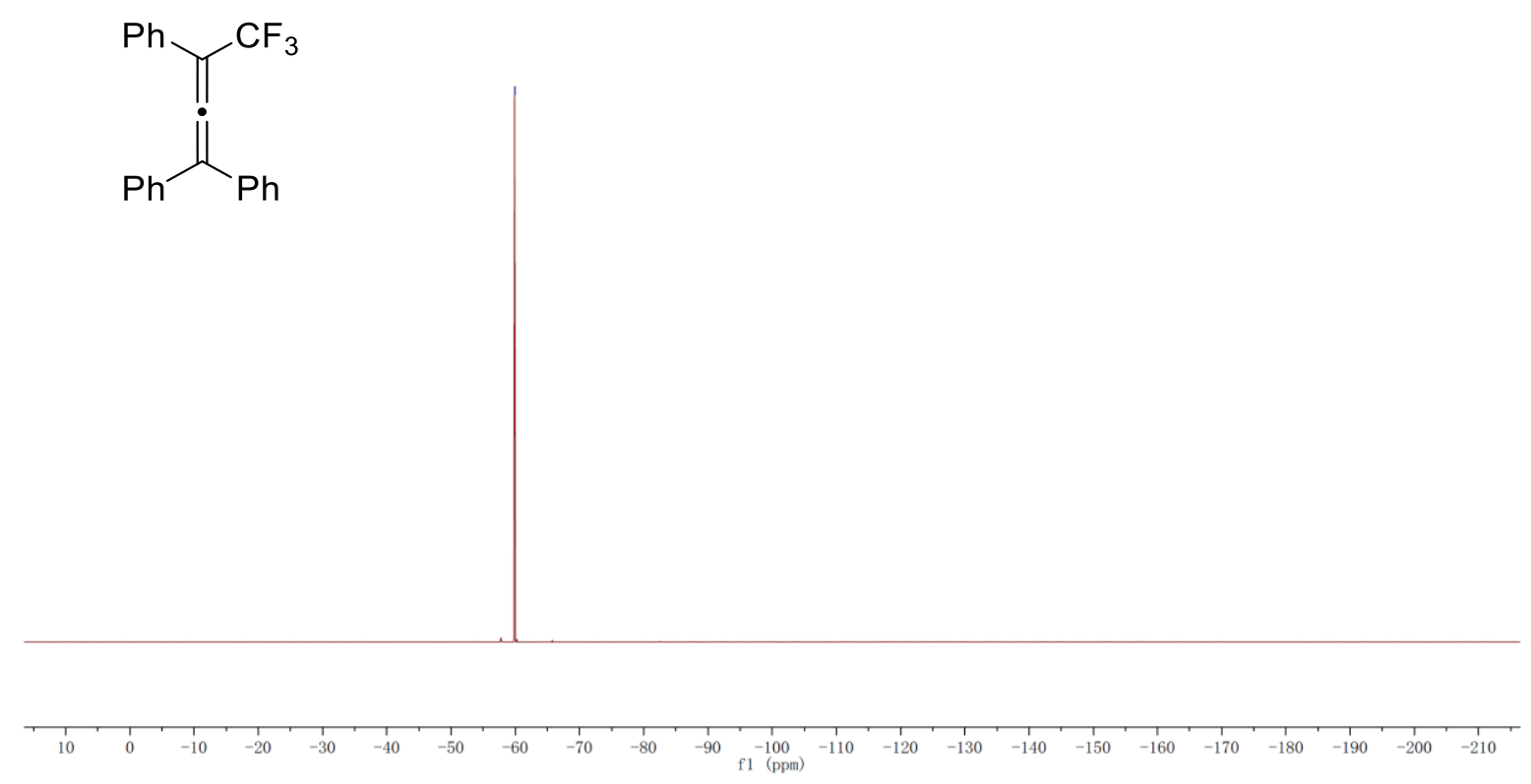


4,4'-(4,4,4-Trifluoro-3-phenylbuta-1,2-diene-1,1-diyl)bis(methylbenzene) (12b)

${ }^{1}$ H NMR (600 MHz, Chloroform- $d$ )

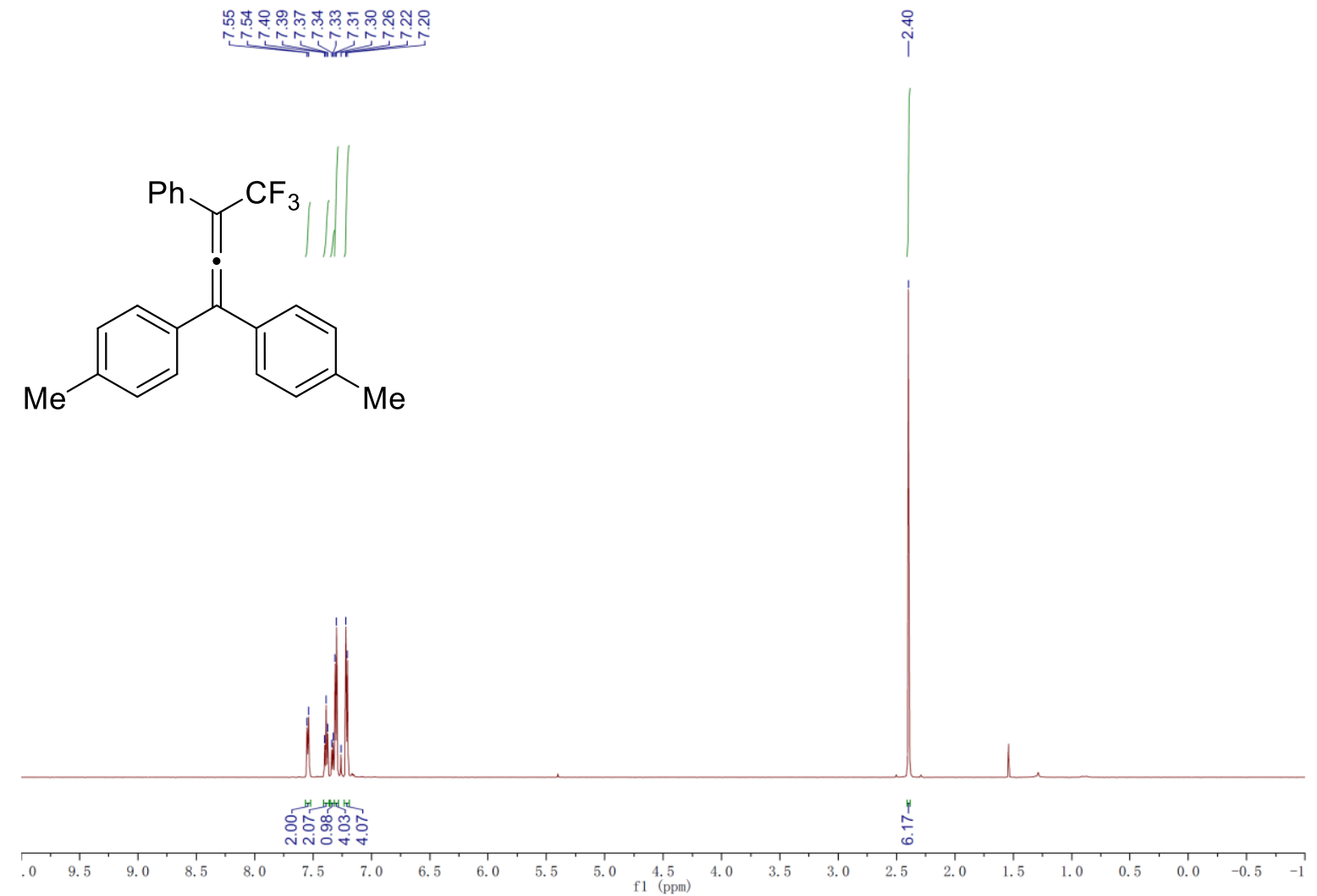

${ }^{13}$ C NMR (151 MHz, Chloroform-d)
นู요

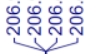

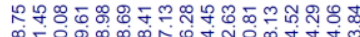

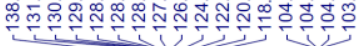<smiles>Cc1ccc(C(=C=C(C(F)(F)F)C(F)(F)F)c2ccc(C)cc2)cc1</smiles>
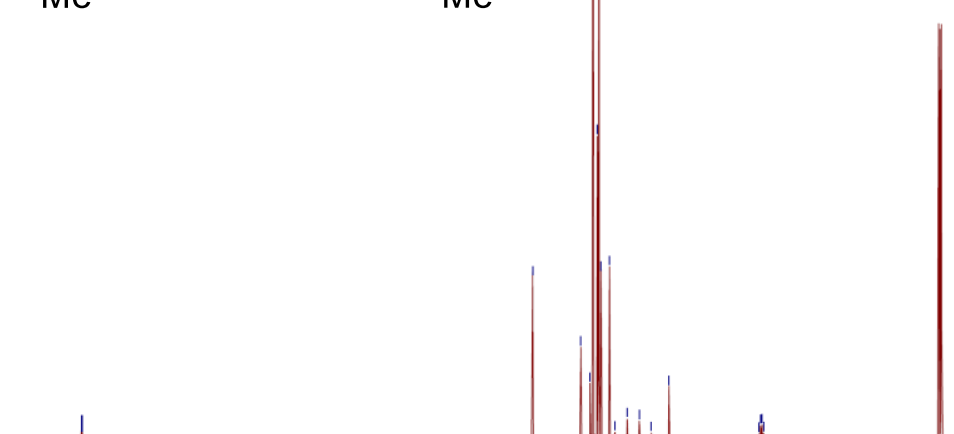

$\begin{array}{rrrrrrrrrrrr}210 & 200 & 190 & 180 & 170 & 160 & 150 & 140 & 130 & 120 & \begin{array}{r}110 \\ \text { f1 }\end{array}\left(\begin{array}{ll}100 \\ (\mathrm{pmm})\end{array}\right.\end{array}$ 
${ }^{19}$ F NMR (565 MHz, Chloroform- $d$ )<smiles>Cc1ccc(C(=C=C(c2ccccc2)C(F)(F)F)c2ccc(C)cc2)cc1</smiles>

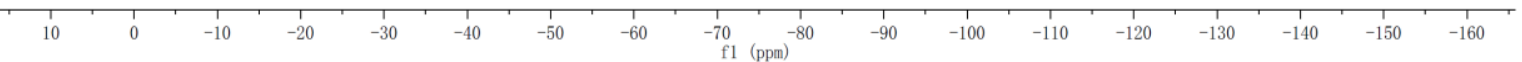


4,4'-(4,4,4-Trifluoro-3-phenylbuta-1,2-diene-1,1-diyl)bis(fluorobenzene) (12c)

${ }^{1}$ H NMR (600 MHz, Chloroform- $d$ )

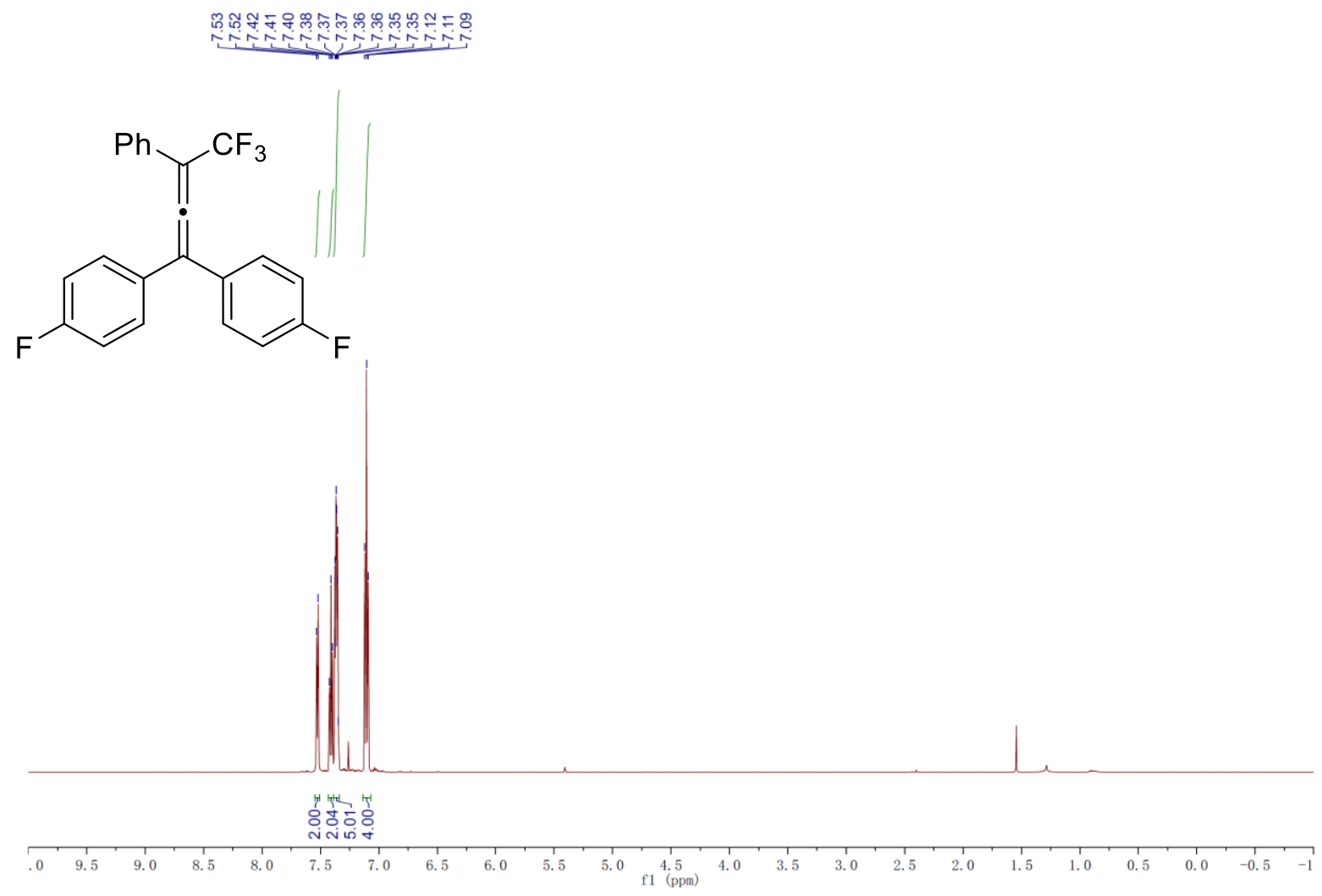

${ }^{13}$ C NMR (151 MHz, Chloroform- $d$ )

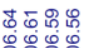

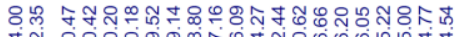

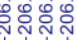
颔<smiles>Fc1ccc(C(=C=C(c2ccc(F)cc2)C(F)(F)F)c2ccc(F)cc2)cc1</smiles>

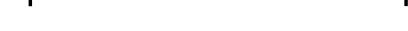

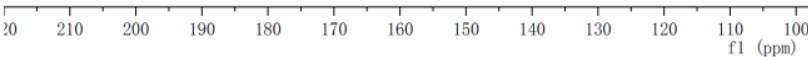


${ }^{19}$ F NMR (565 MHz, Chloroform- $d$ )

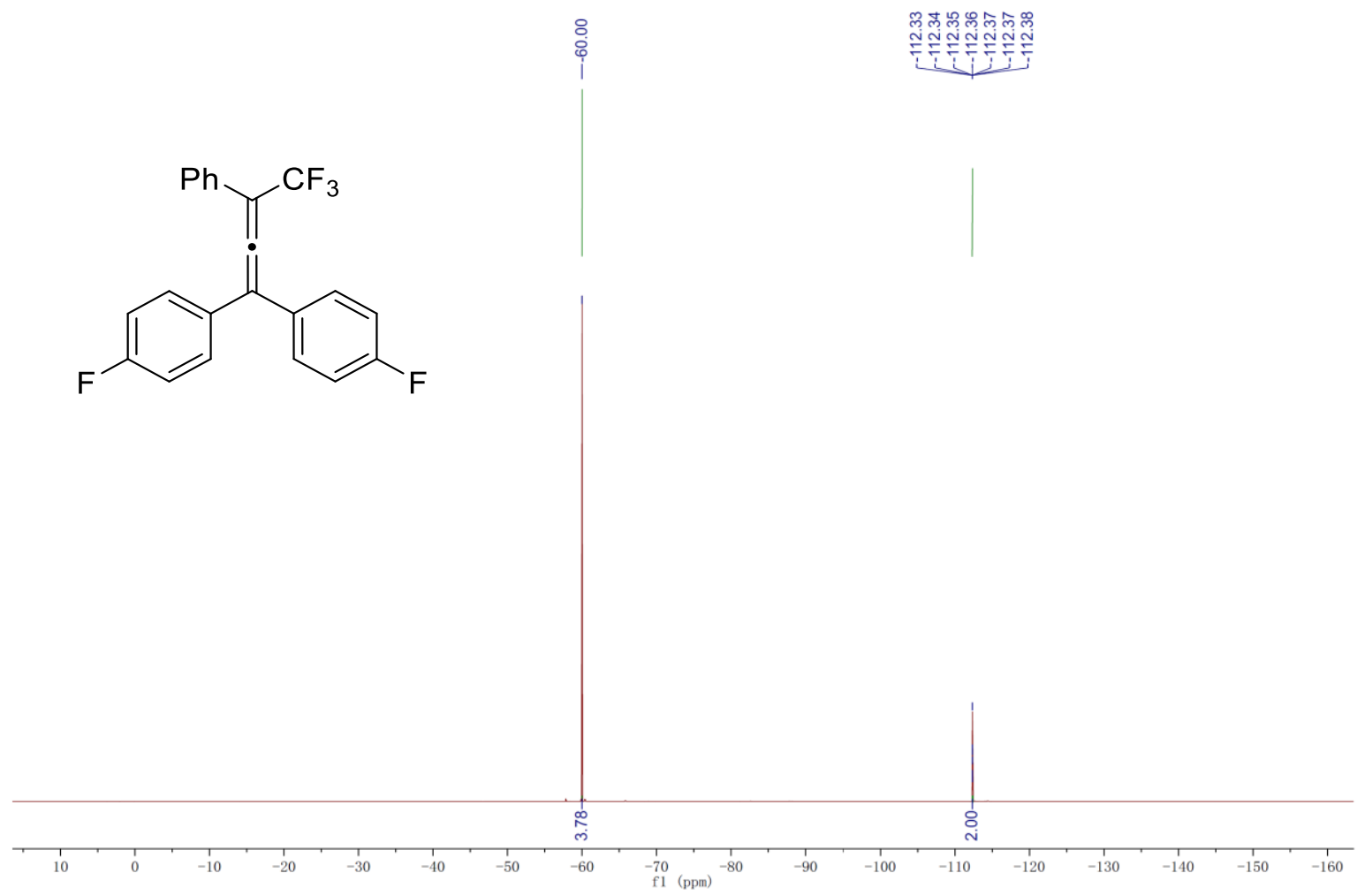


4,4'-(4,4,4-Trifluoro-3-phenylbuta-1,2-diene-1,1-diyl)bis(chlorobenzene) (12d)

${ }^{1}$ H NMR (600 MHz, Chloroform- $d$ )

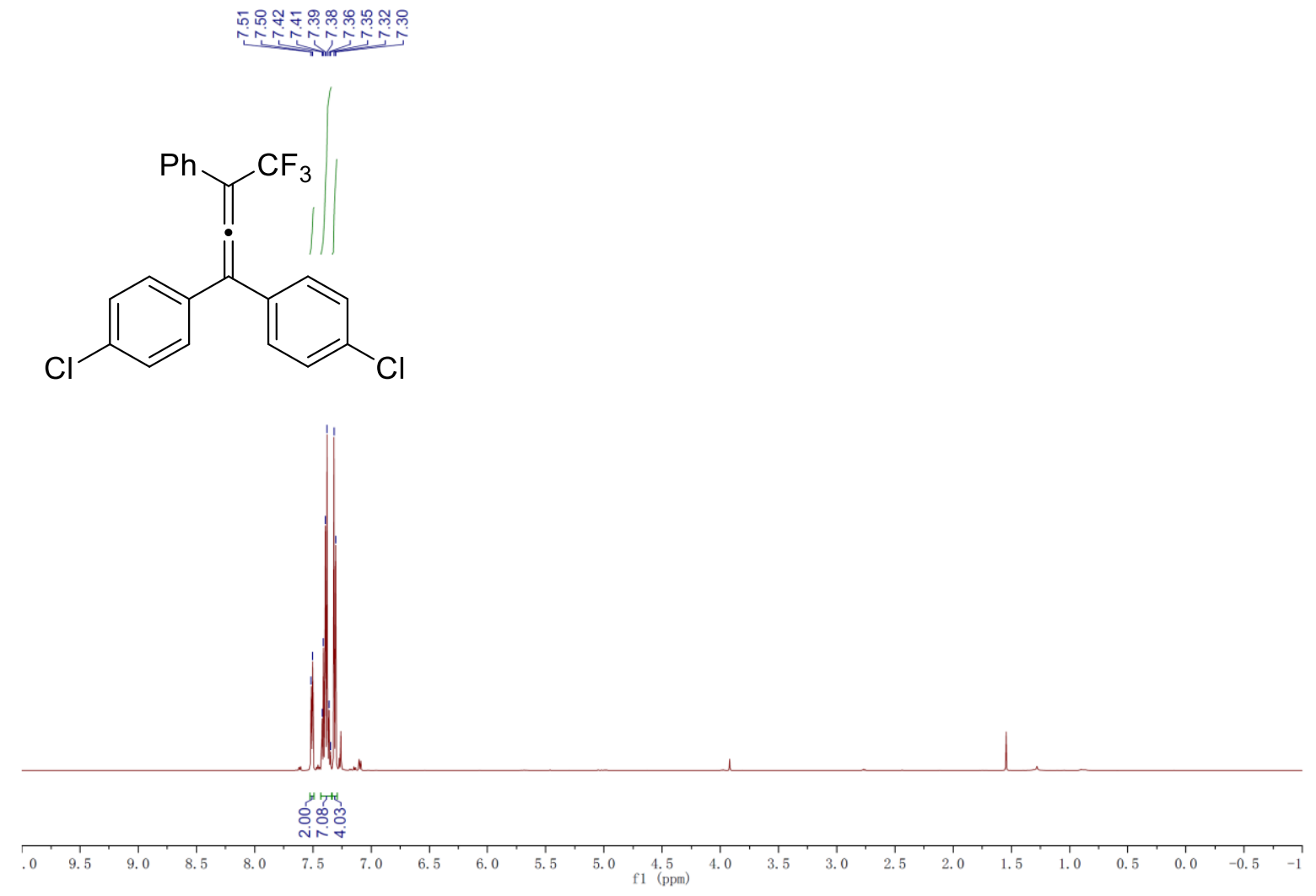

${ }^{13}$ C NMR (151 MHz, Chloroform- $d$ )

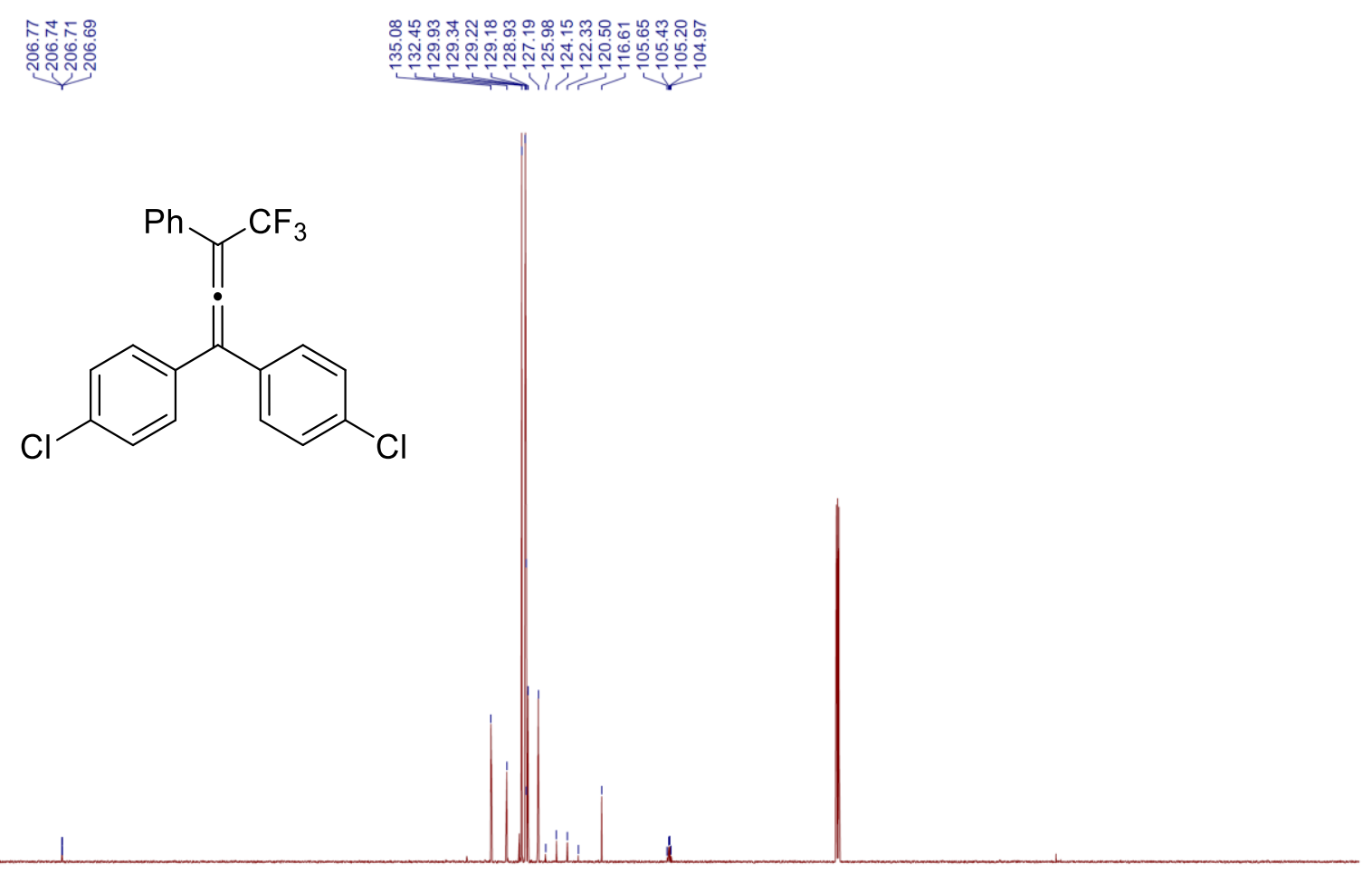

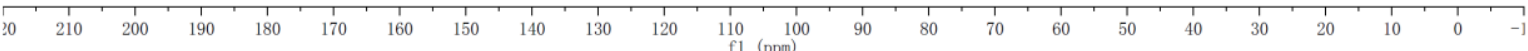


${ }^{19}$ F NMR (565 MHz, Chloroform- $d$ )

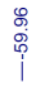<smiles>FC(F)(F)C(=NC(c1ccc(Cl)cc1)c1ccc(Cl)cc1)c1ccccc1</smiles>

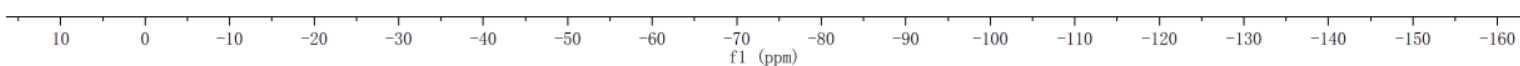


4,4'-(4,4,4-Trifluoro-3-phenylbuta-1,2-diene-1,1-diyl)bis(methoxybenzene) (12e)

${ }^{1}$ H NMR (600 MHz, Chloroform- $d$ )

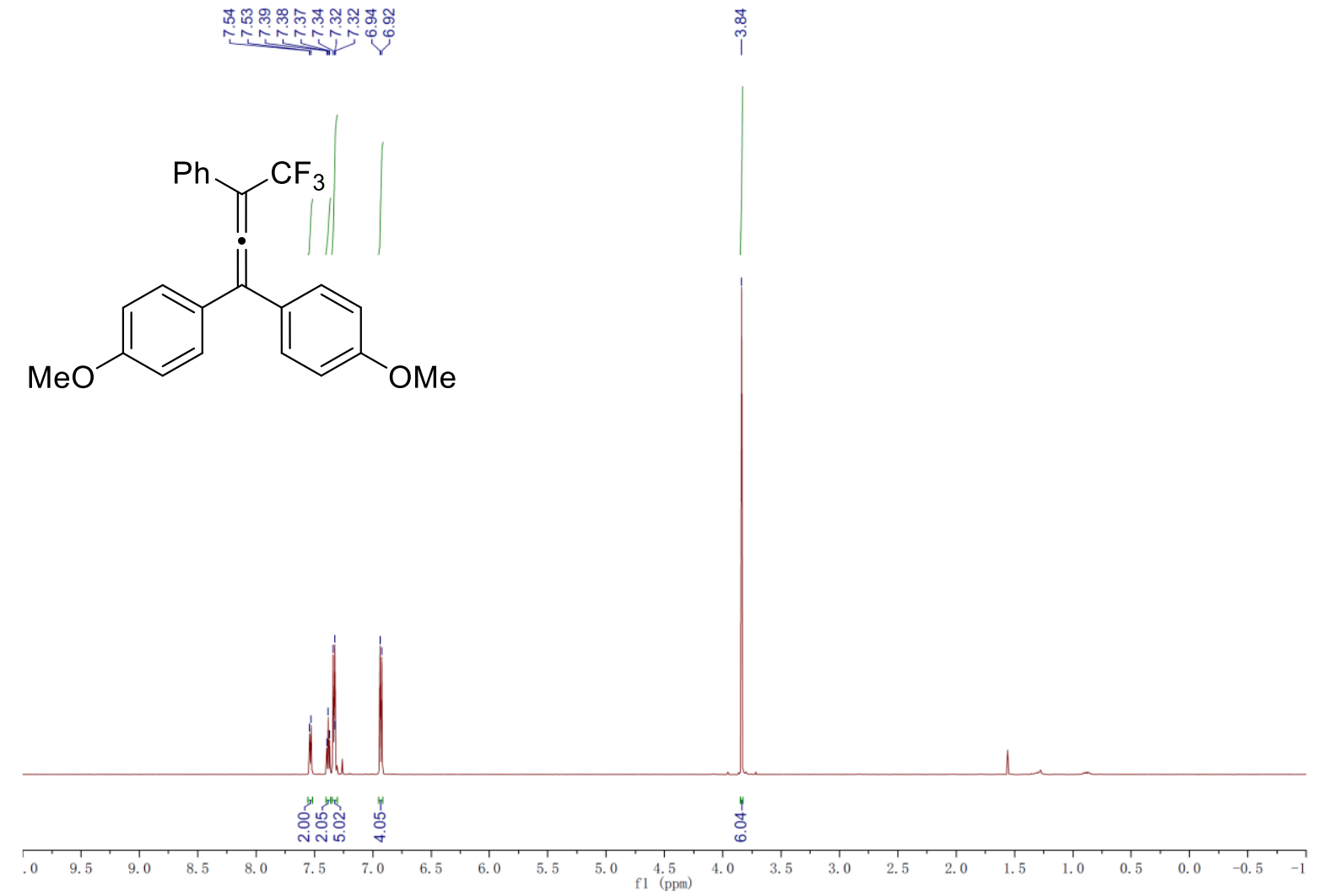

${ }^{13}$ C NMR (151 MHz, Chloroform-d)

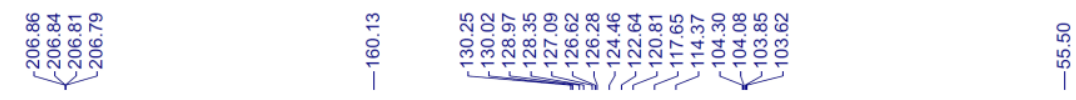

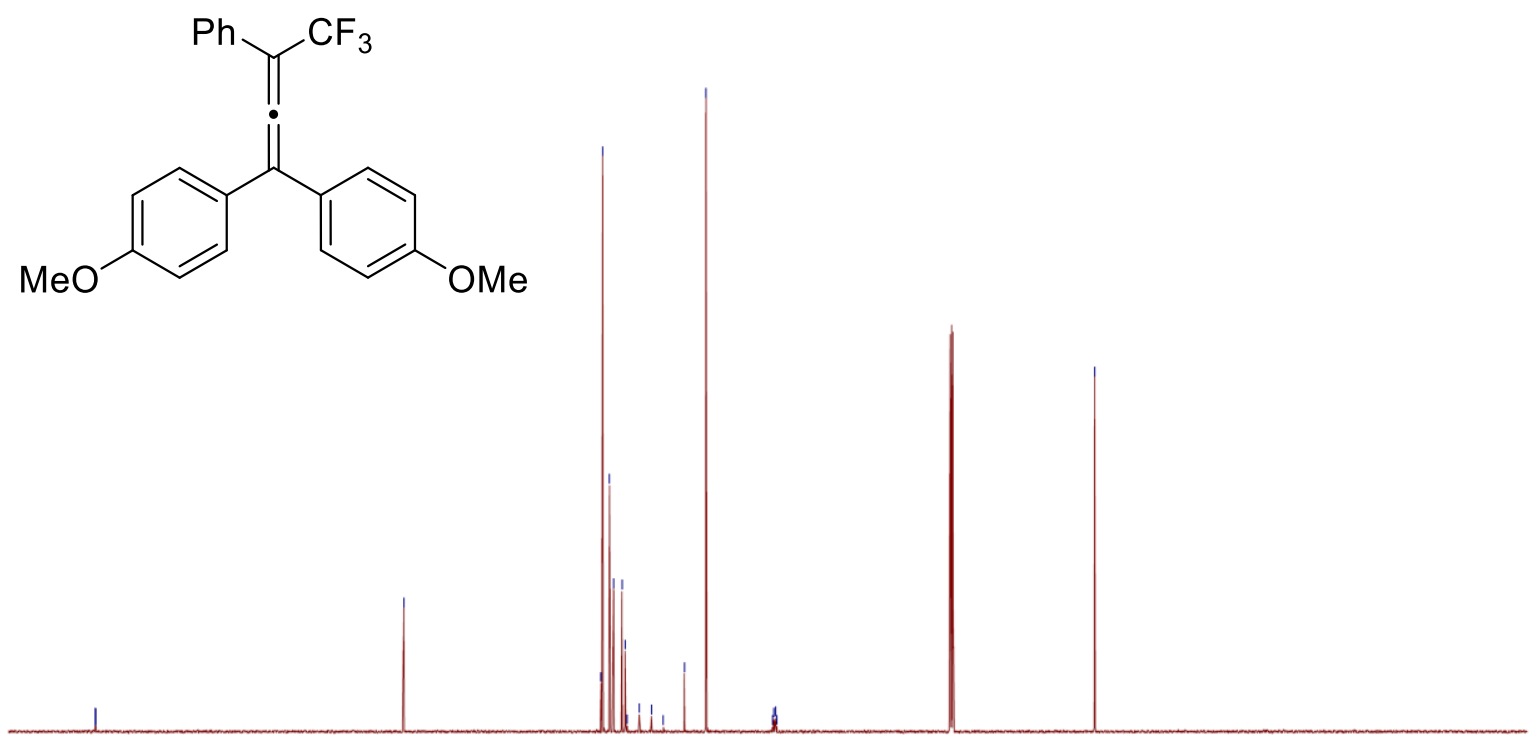

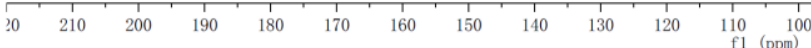


${ }^{19}$ F NMR (565 MHz, Chloroform- $d$ )

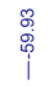

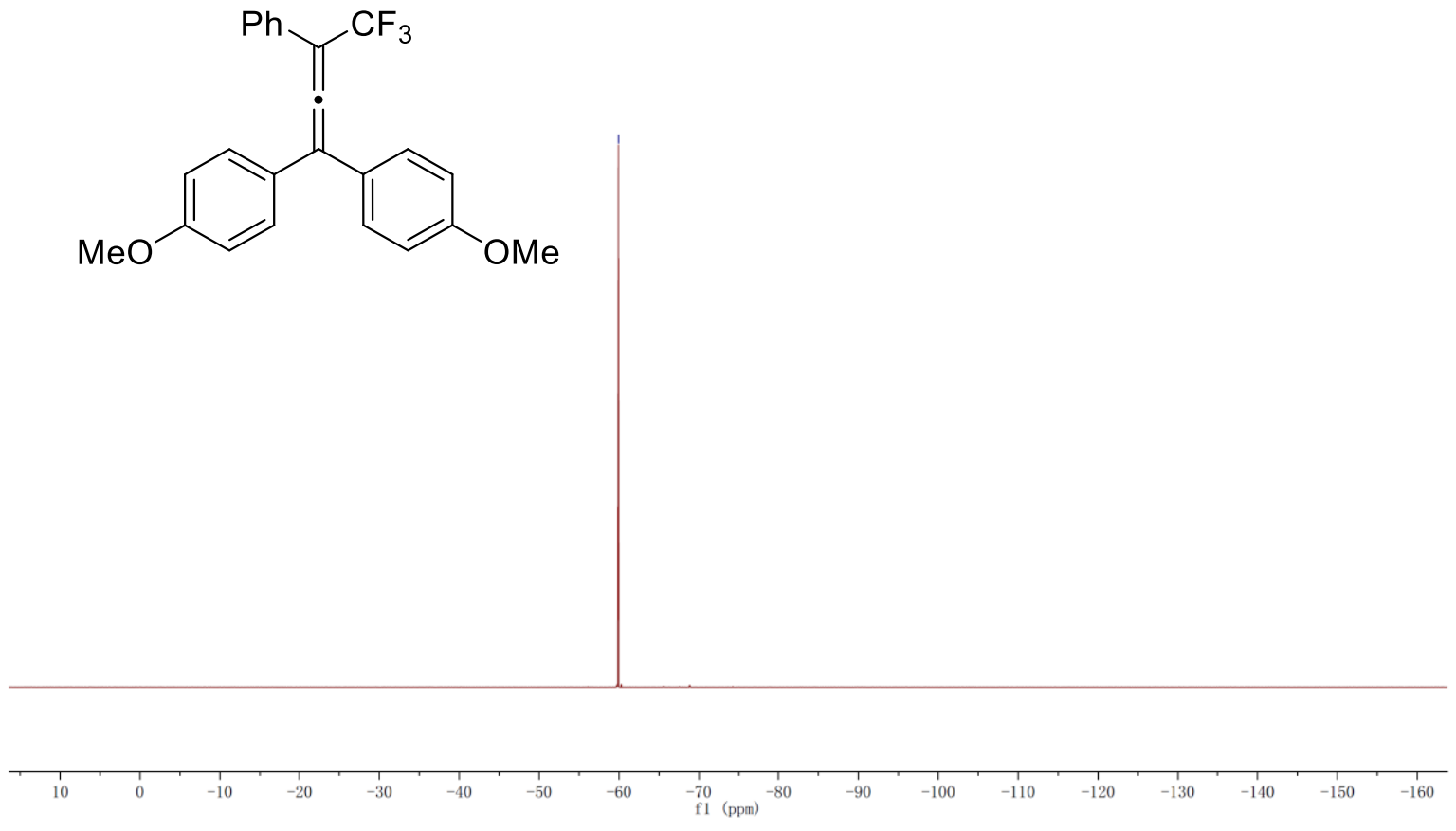


(4,4,4-Trifluoro-1-( $p$-tolyl)buta-1,2-diene-1,3-diyl)dibenzene (12f)

${ }^{1}$ H NMR (600 MHz, Chloroform- $d$ )

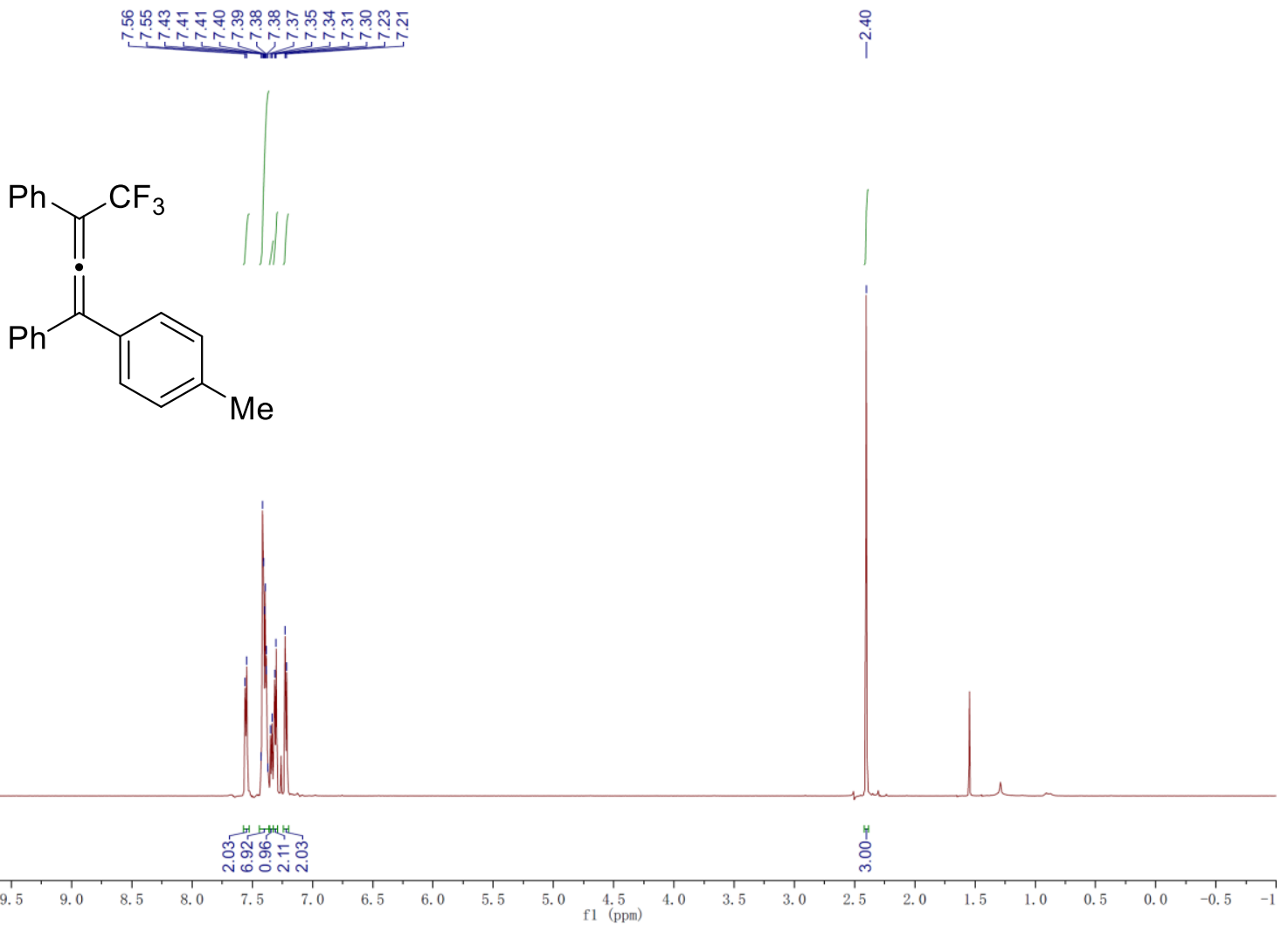

${ }^{13}$ C NMR (151 MHz, Chloroform- $\left.d\right)$

\section{\%}

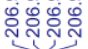

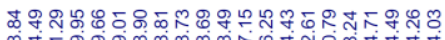

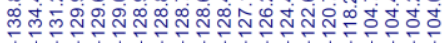<smiles>Cc1ccc(C(=C(c2ccccc2)C(F)(F)F)c2ccccc2)cc1</smiles> 
${ }^{19}$ F NMR (565 MHz, Chloroform- $d$ )

क़े<smiles>Cc1ccc(/C(=C/C(=C(/P)C(F)(F)F)c2ccccc2)c2ccccc2)cc1</smiles>

\begin{tabular}{rllllllllllllllllllllllllll}
1 & 1 & 1 & 1 & -10 & -20 & -30 & -40 & -50 & -60 & -70 & -80 & -90 & -100 & -110 & -120 & -130 & -140 & -150 & -160 & -170 & -180 & -190 & -200 & -210 \\
\hline
\end{tabular} 
(4,4,4-Trifluoro-1-(4-fluorophenyl)buta-1,2-diene-1,3-diyl)dibenzene (12g)

${ }^{1}$ H NMR (600 MHz, Chloroform- $d$ )

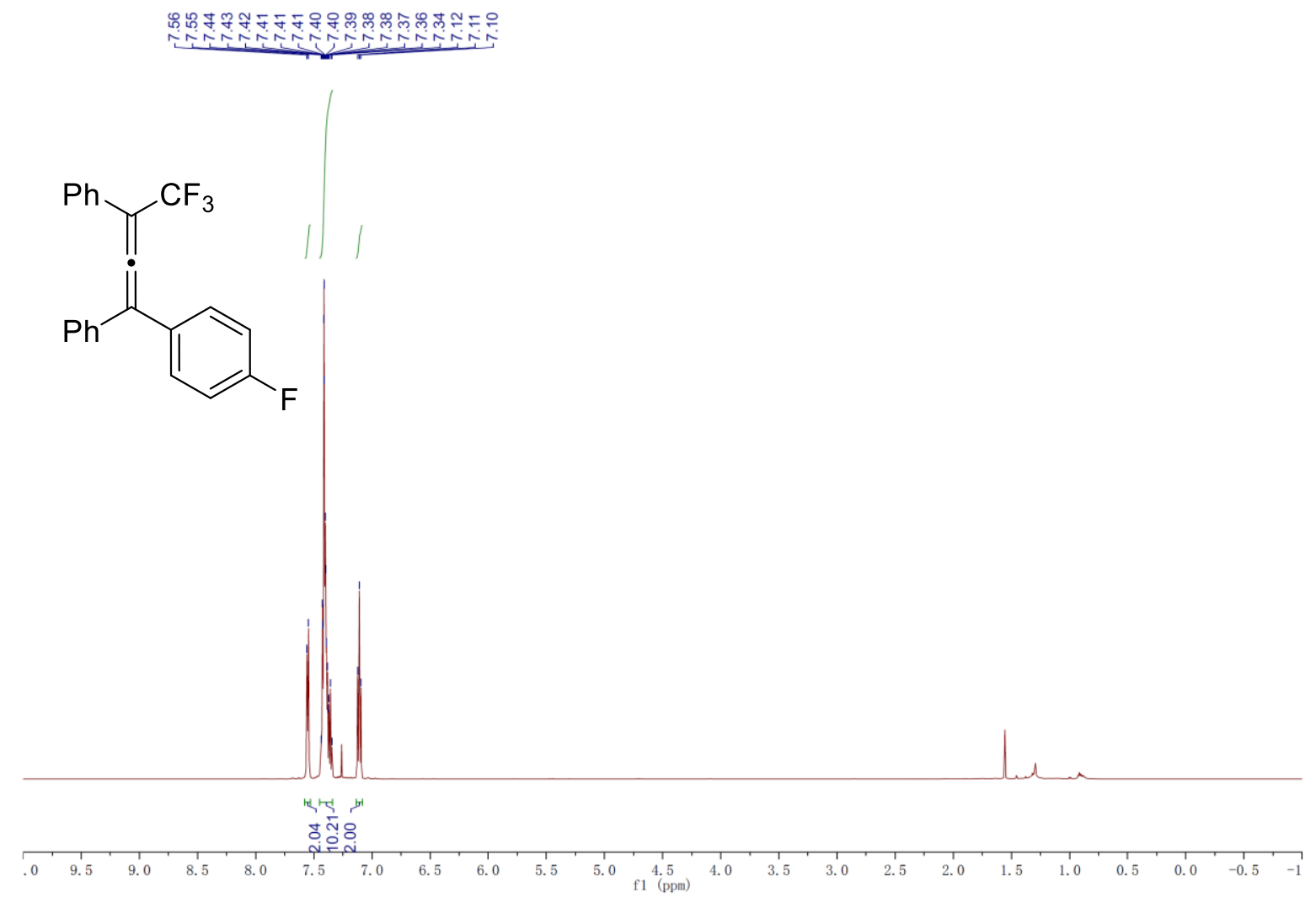

${ }^{13}$ C NMR (151 MHz, Chloroform-d)

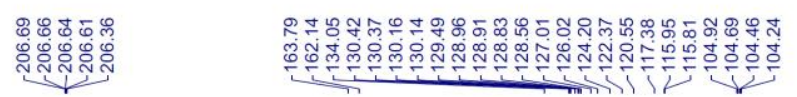<smiles>Fc1ccc(C(=C=C(c2ccccc2)C(F)(F)F)c2ccccc2)cc1</smiles>
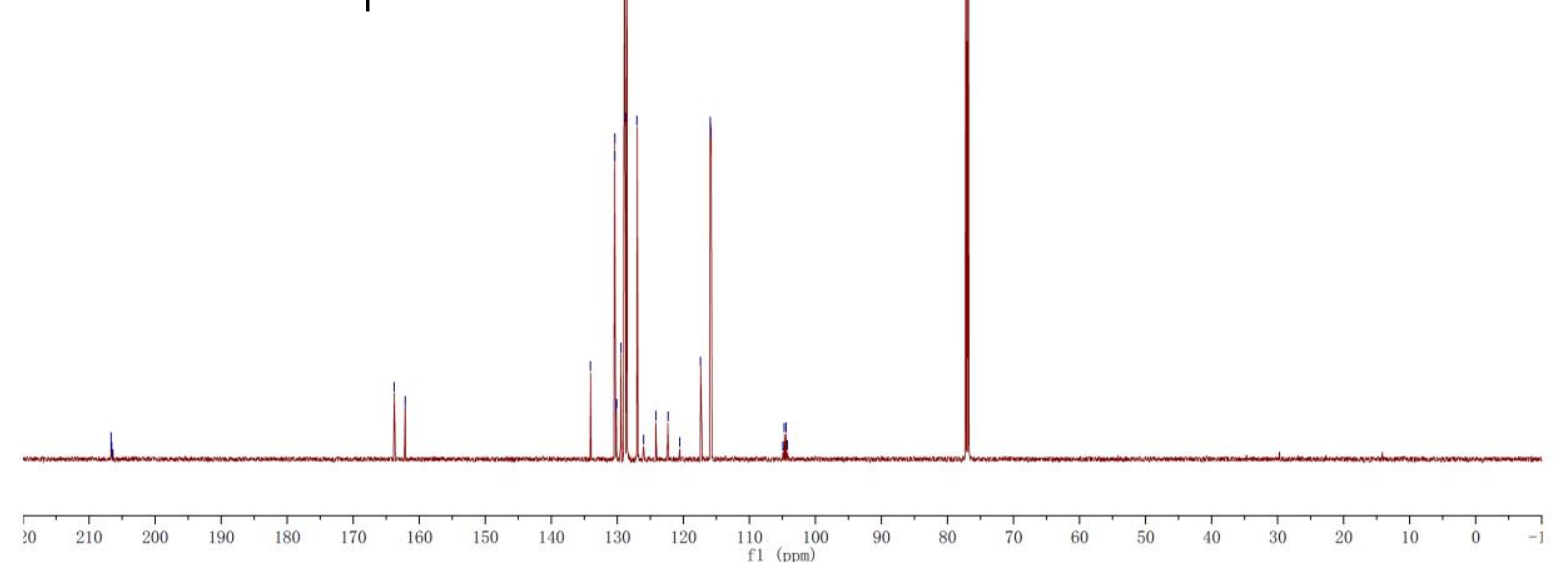
${ }^{19}$ F NMR (565 MHz, Chloroform- $d$ )

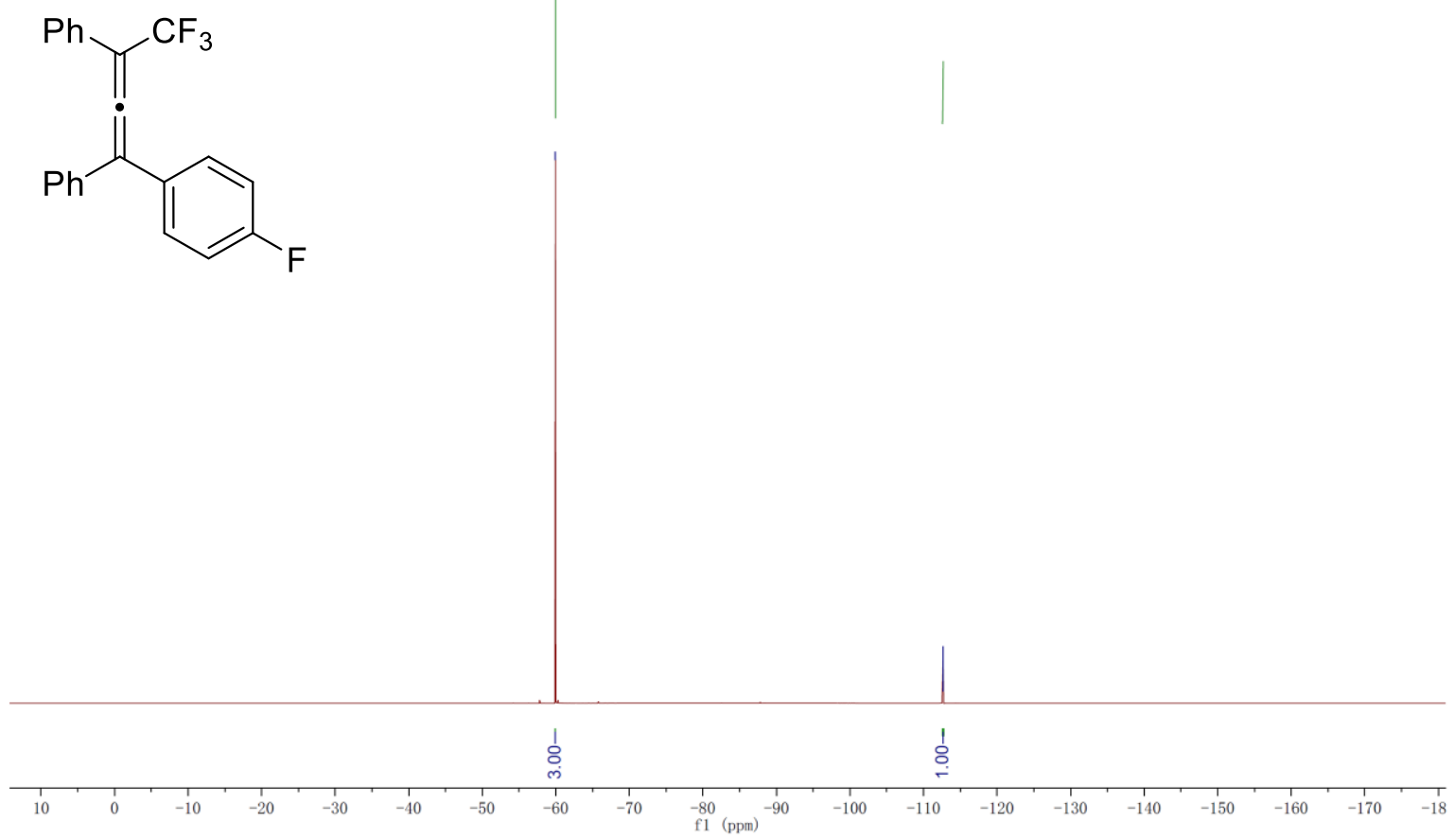


(1-(4-Chlorophenyl)-4,4,4-trifluorobuta-1,2-diene-1,3-diyl)dibenzene (12h)

${ }^{1}$ H NMR (600 MHz, Chloroform- $d$ )

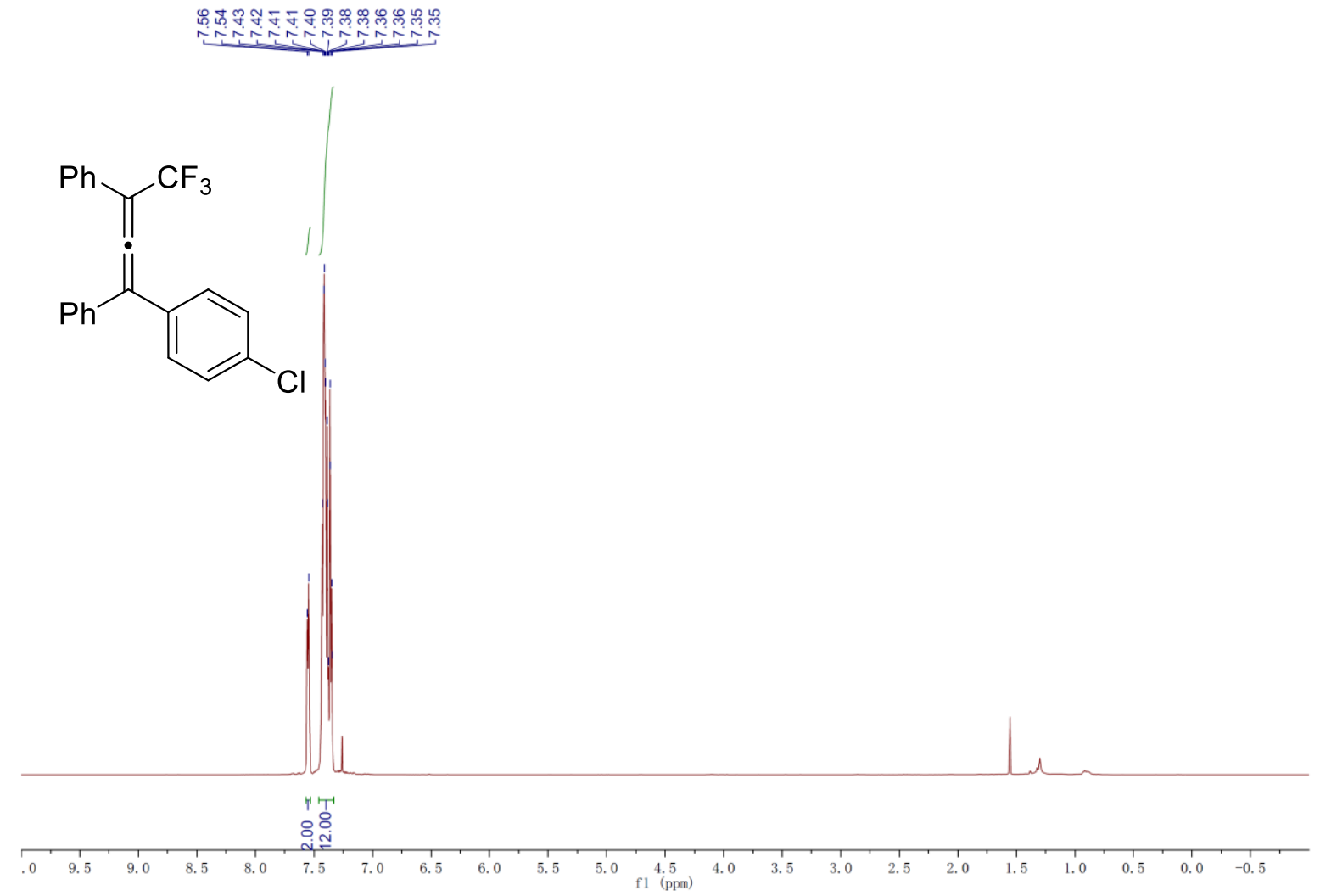

${ }^{13}$ C NMR (151 MHz, Chloroform-d)

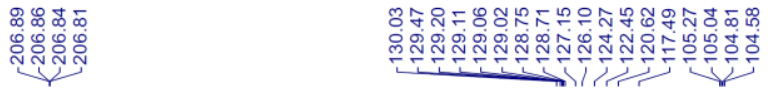<smiles>FC(F)(F)C(=C=C(c1ccccc1)c1ccc(Cl)cc1)c1ccccc1</smiles>

$\begin{array}{lllllllllllll}20 & 210 & 200 & 190 & 180 & 170 & 160 & 150 & 140 & 130 & 120 & 110 \\ \mathrm{f} 1(\mathrm{ppm}) & 100\end{array}$ 
${ }^{19}$ F NMR (565 MHz, Chloroform- $d$ )<smiles>FC(F)(F)C(=C=C(c1ccccc1)c1ccccc1)c1ccccc1</smiles>

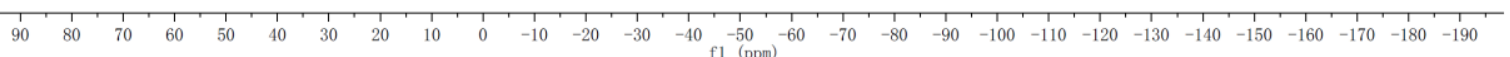


(1-(4-Bromophenyl)-4,4,4-trifluorobuta-1,2-diene-1,3-diyl)dibenzene (12i)

${ }^{1}$ H NMR (600 MHz, Chloroform- $d$ )

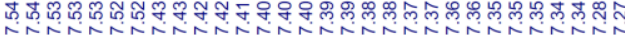

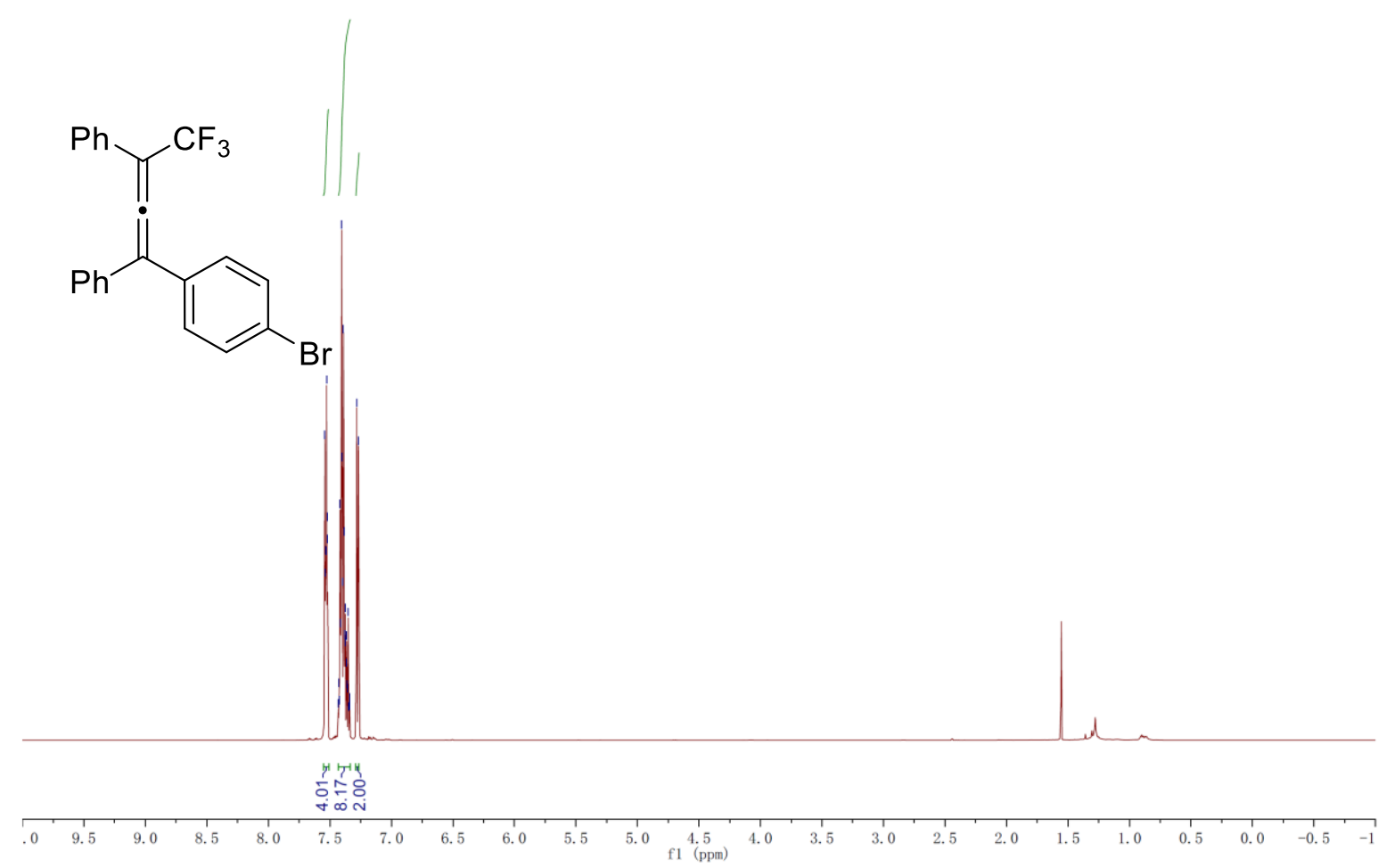

${ }^{13}$ C NMR (151 MHz, Chloroform- $d$ )

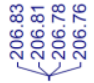

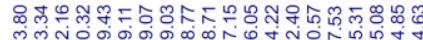

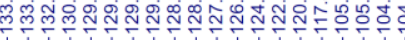<smiles>FC(F)(F)C(=C=C(c1ccccc1)c1ccccc1)c1ccccc1</smiles> 
${ }^{19}$ F NMR (565 MHz, Chloroform- $d$ )<smiles>FC(F)(F)C(=Cc1ccc(Br)cc1)c1ccccc1</smiles>

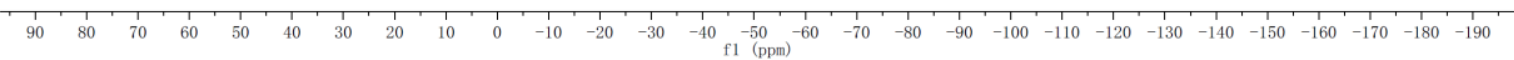


(4,4,4-Trifluoro-1-(4-methoxyphenyl)buta-1,2-diene-1,3-diyl)dibenzene (12j)

${ }^{1}$ H NMR (600 MHz, Chloroform- $d$ )

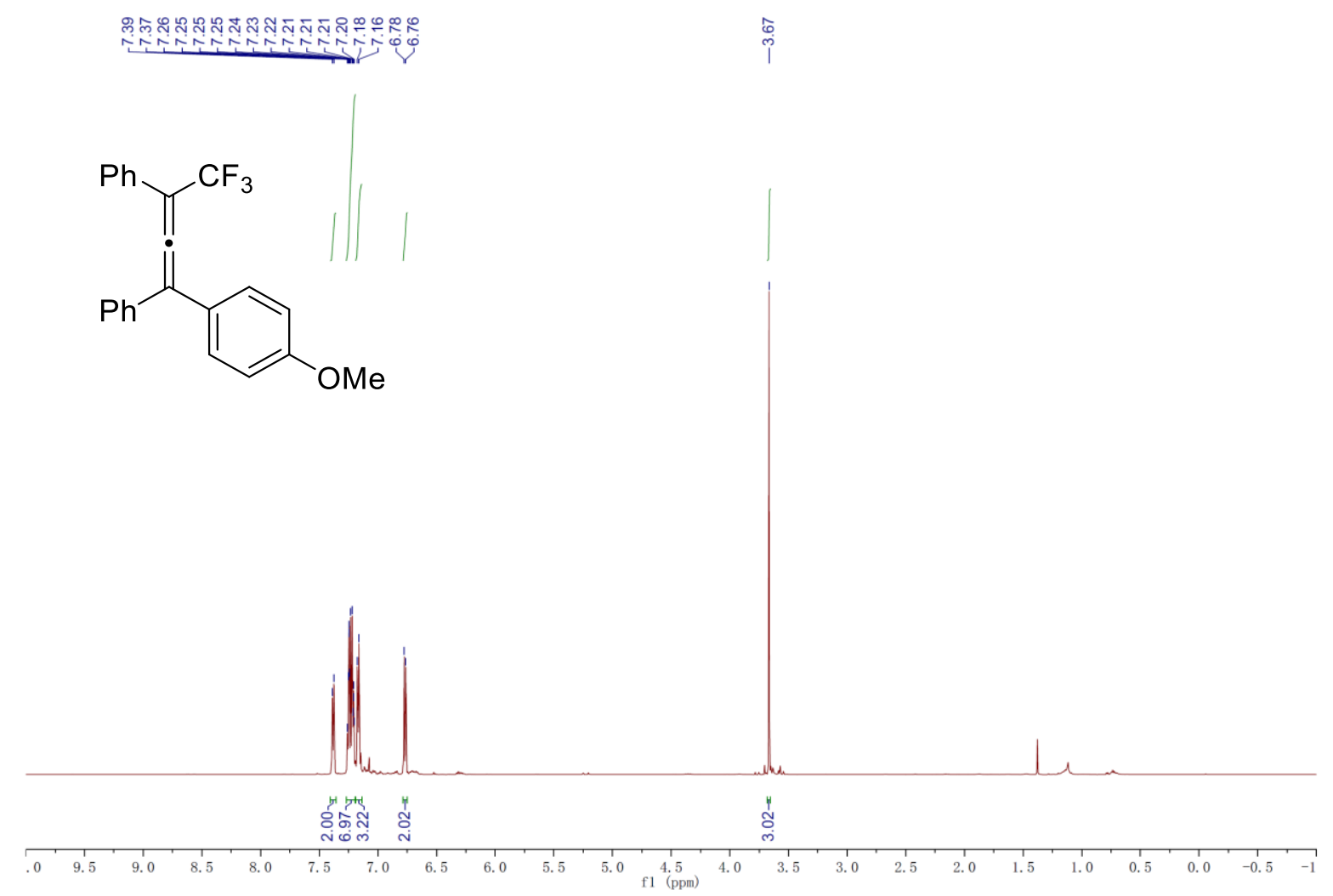

${ }^{13}$ C NMR (151 MHz, Chloroform- $d$ )
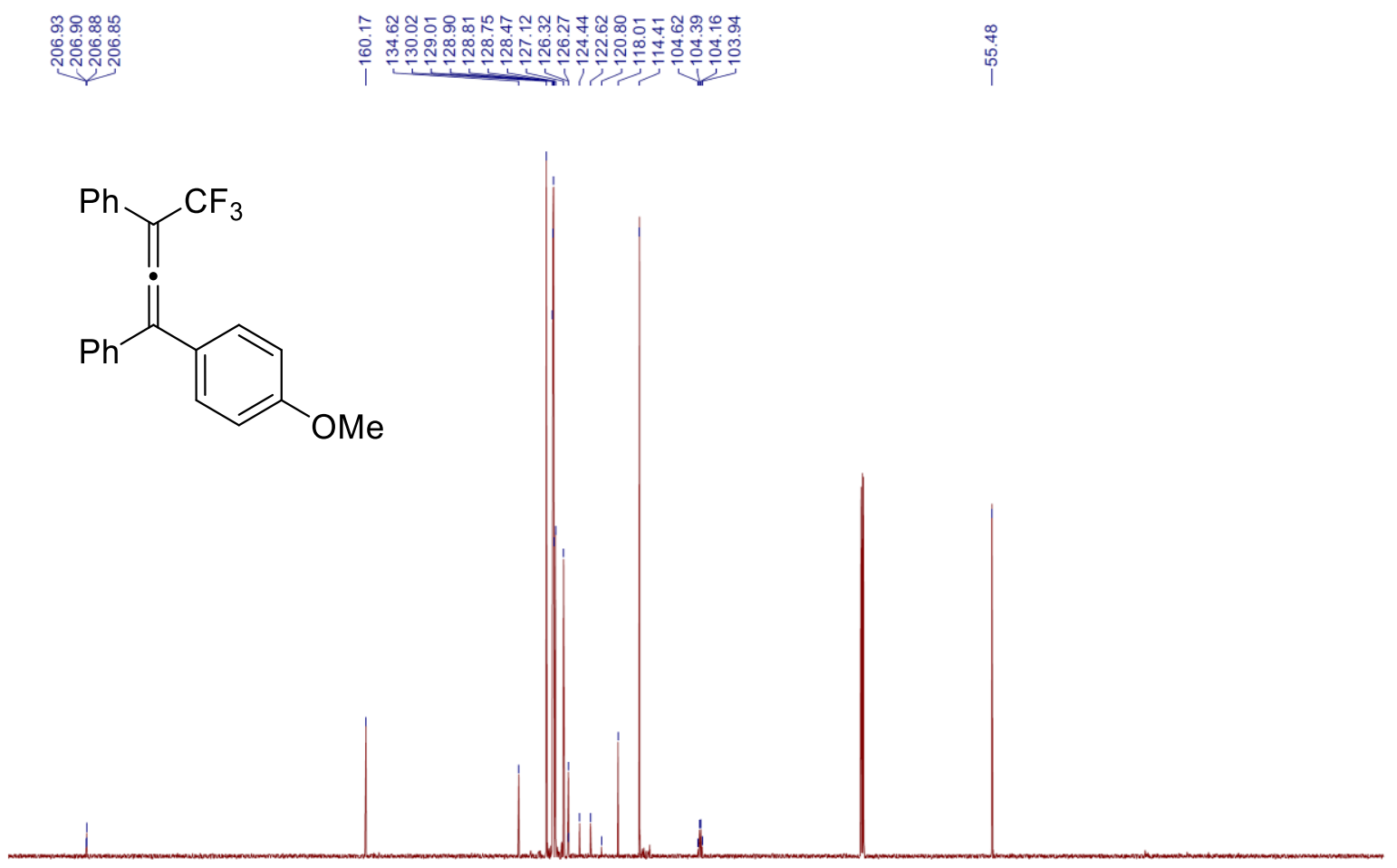

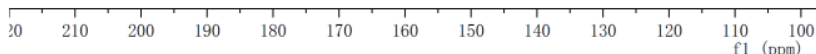


${ }^{19}$ F NMR (565 MHz, Chloroform- $d$ )<smiles>COc1ccc(/C(=C/c2ccccc2)C(F)(F)F)cc1</smiles>

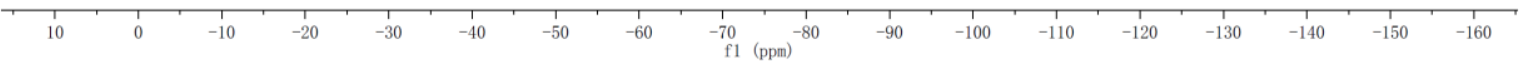


4-(4,4,4-Trifluoro-1,3-diphenylbuta-1,2-dien-1-yl)-1,1'-biphenyl (12k)

${ }^{1}$ H NMR $(600 \mathrm{MHz}$, Chloroform- $d$ )

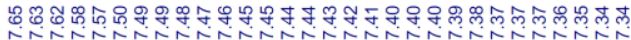

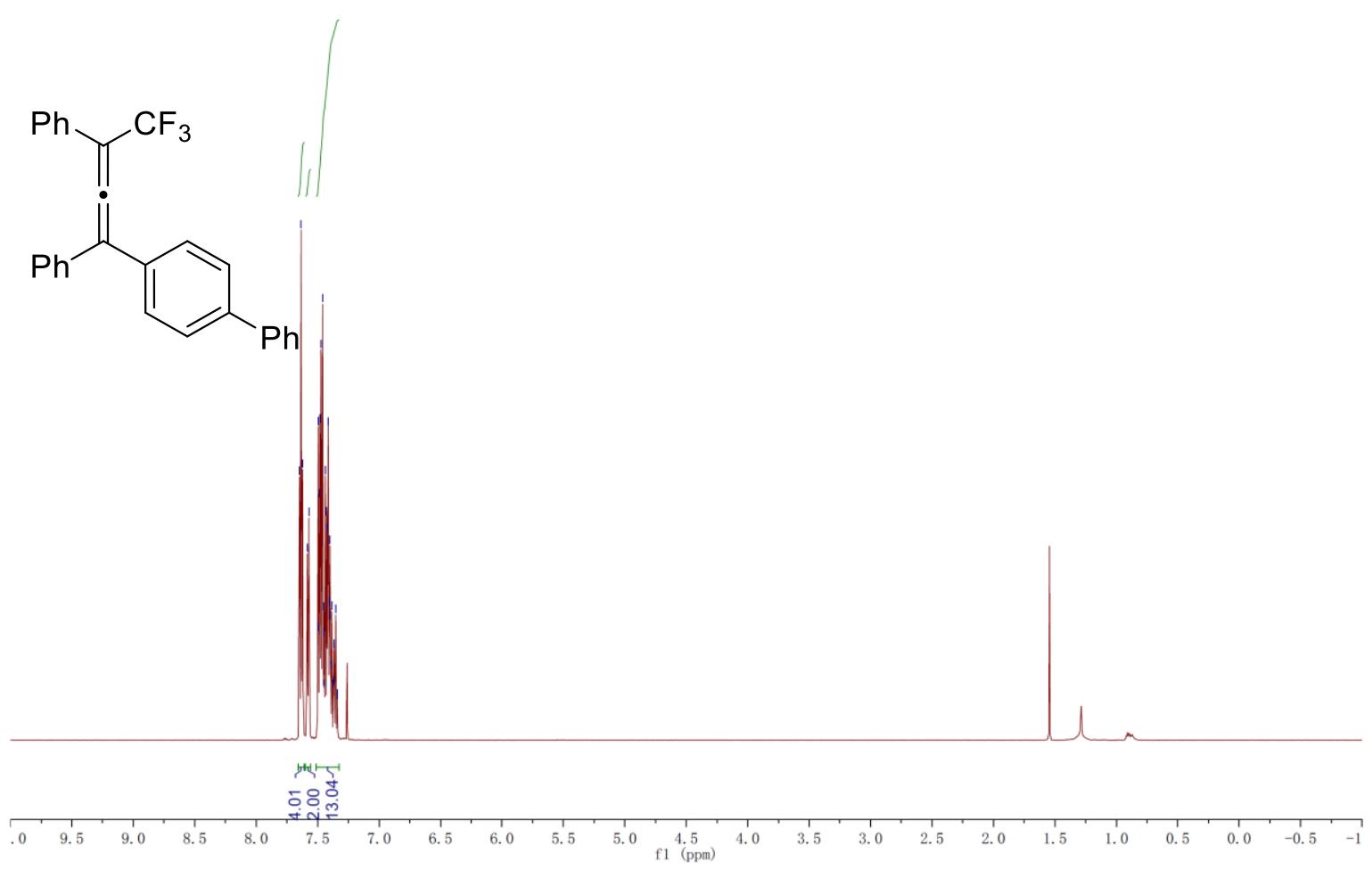

${ }^{13}$ C NMR (151 MHz, Chloroform- $d$ )

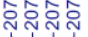

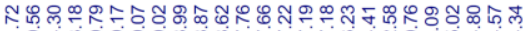

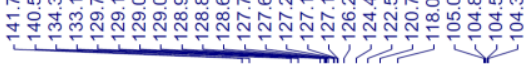

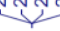

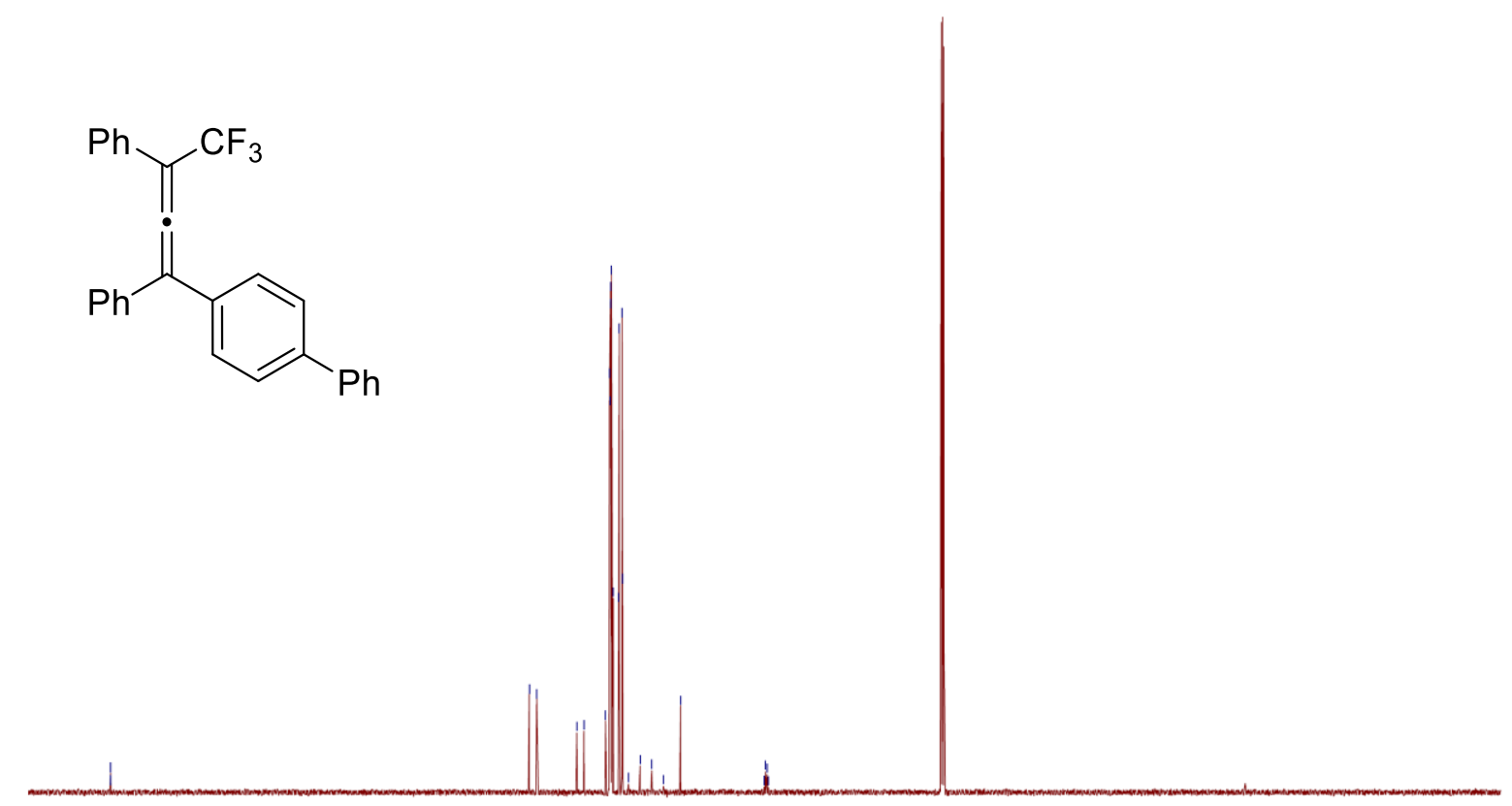

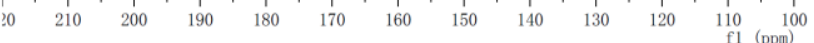


${ }^{19}$ F NMR (565 MHz, Chloroform- $d$ )

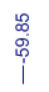

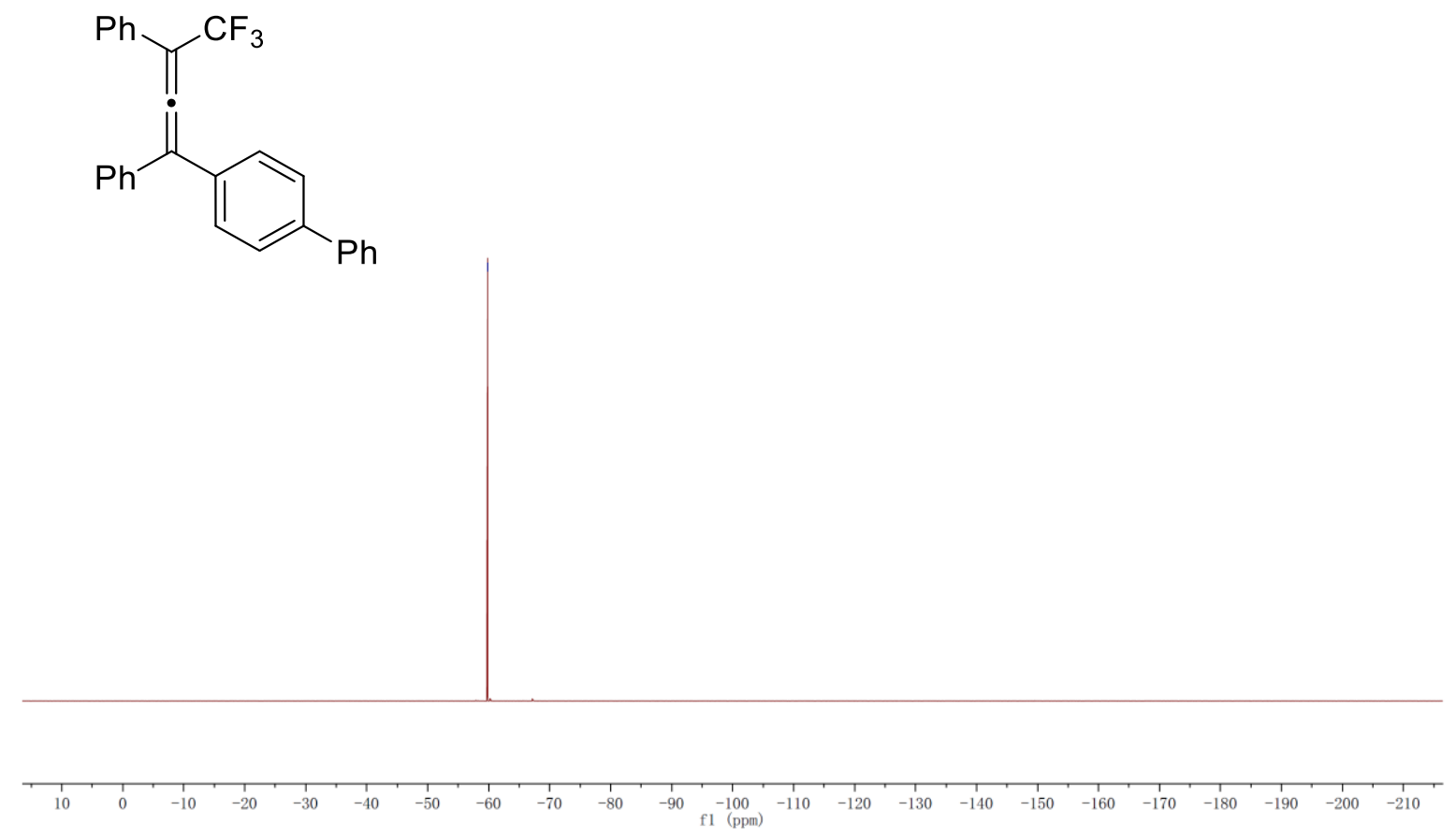


(4,4,4-Trifluoro-1-( $m$-tolyl)buta-1,2-diene-1,3-diyl)dibenzene (12l)

${ }^{1}$ H NMR (600 MHz, Chloroform- $d$ )

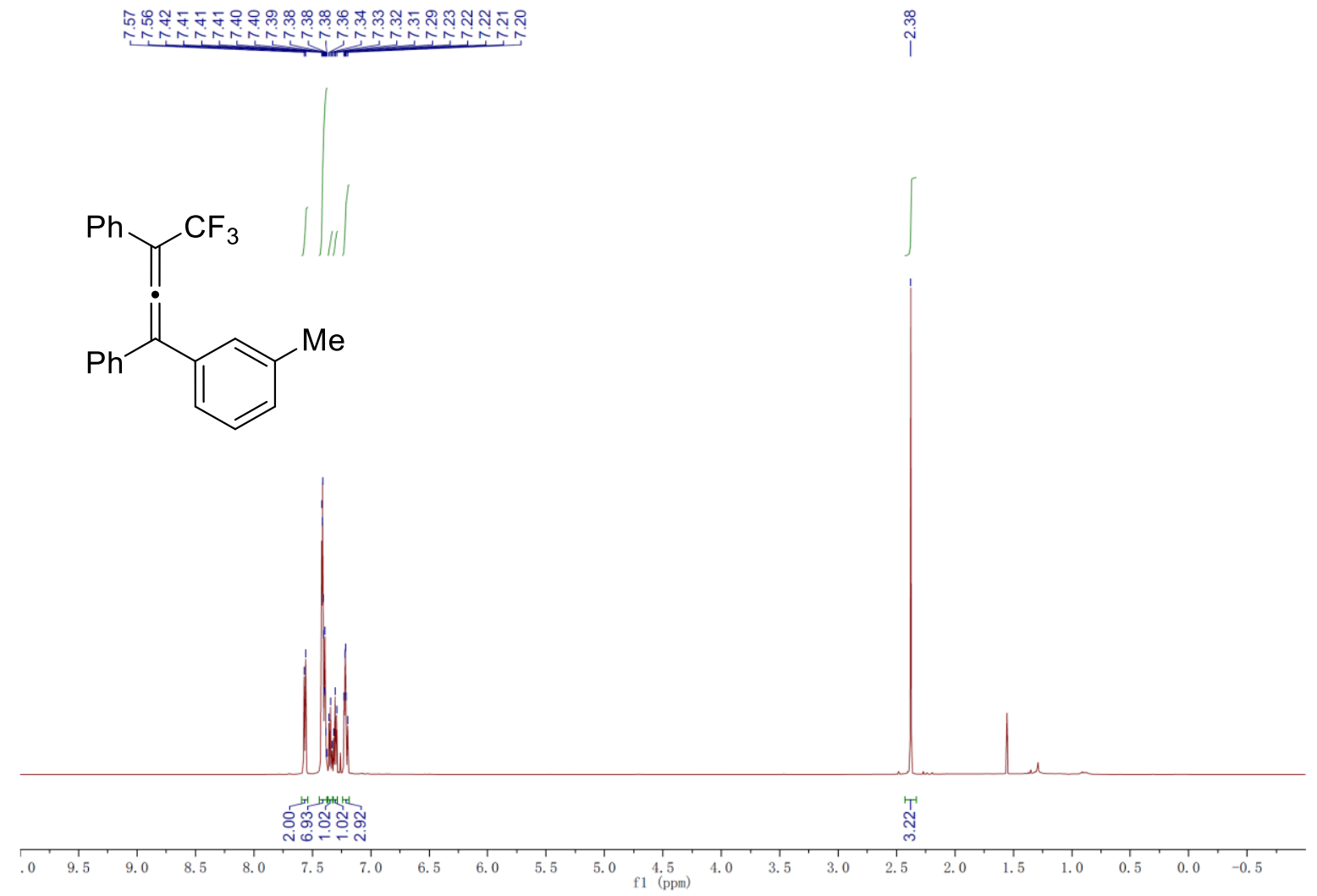

${ }^{13}$ C NMR (151 MHz, Chloroform-d)

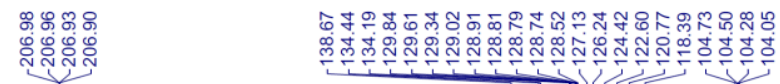

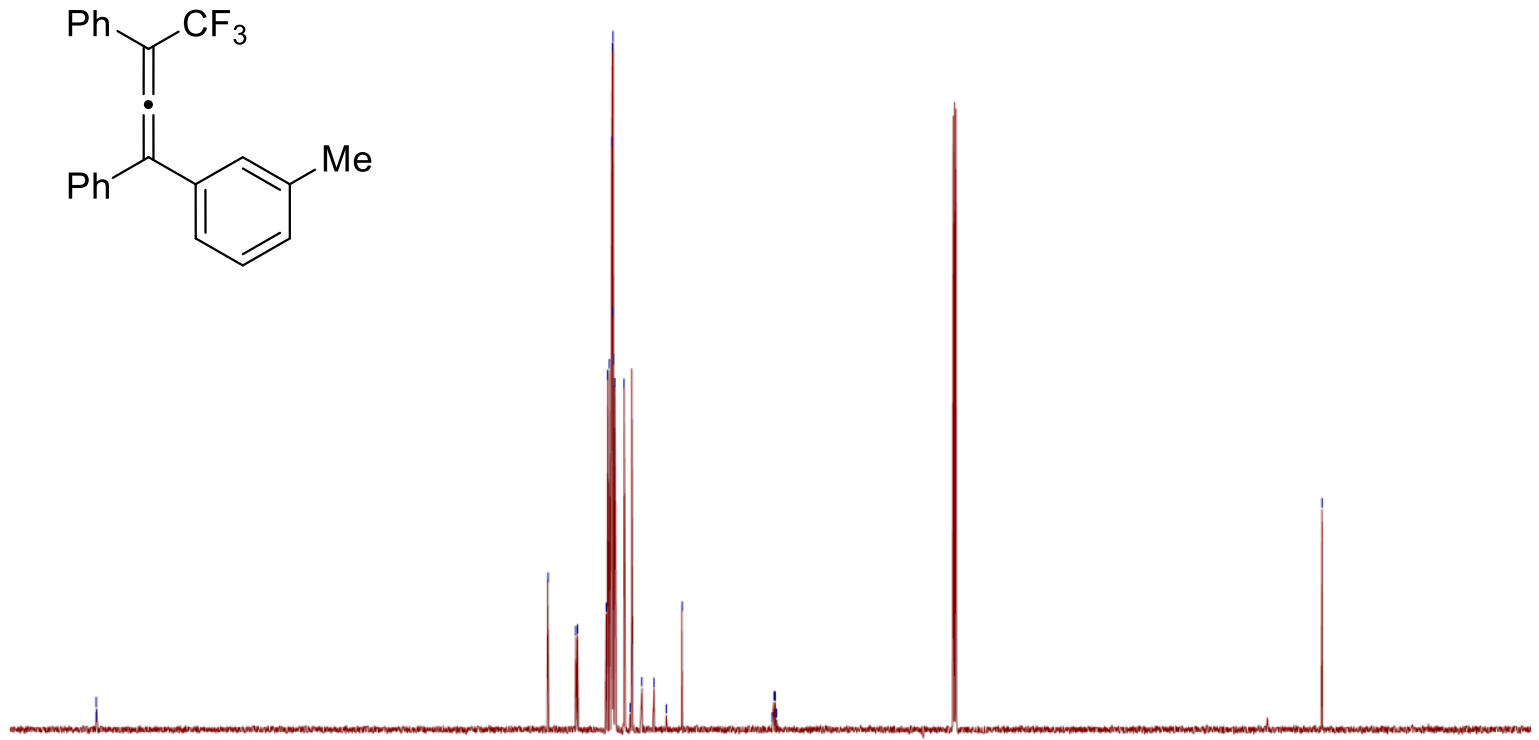


${ }^{19}$ F NMR (565 MHz, Chloroform- $d$ )<smiles>Cc1cccc(C(=C=C(c2ccccc2)C(F)(F)F)c2ccccc2)c1</smiles>

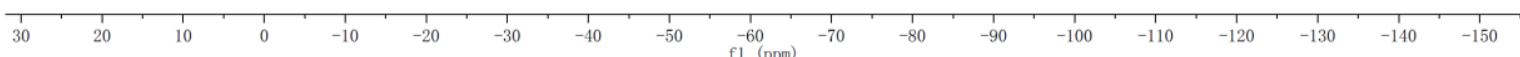


(4,4,4-Trifluoro-1-(o-tolyl)buta-1,2-diene-1,3-diyl)dibenzene (12m)

${ }^{1}$ H NMR (600 MHz, Chloroform- $d$ )

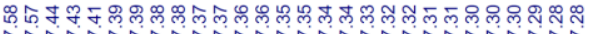

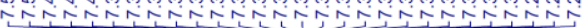<smiles>Cc1ccccc1C(=C=C(c1ccccc1)C(F)(F)F)c1ccccc1</smiles>

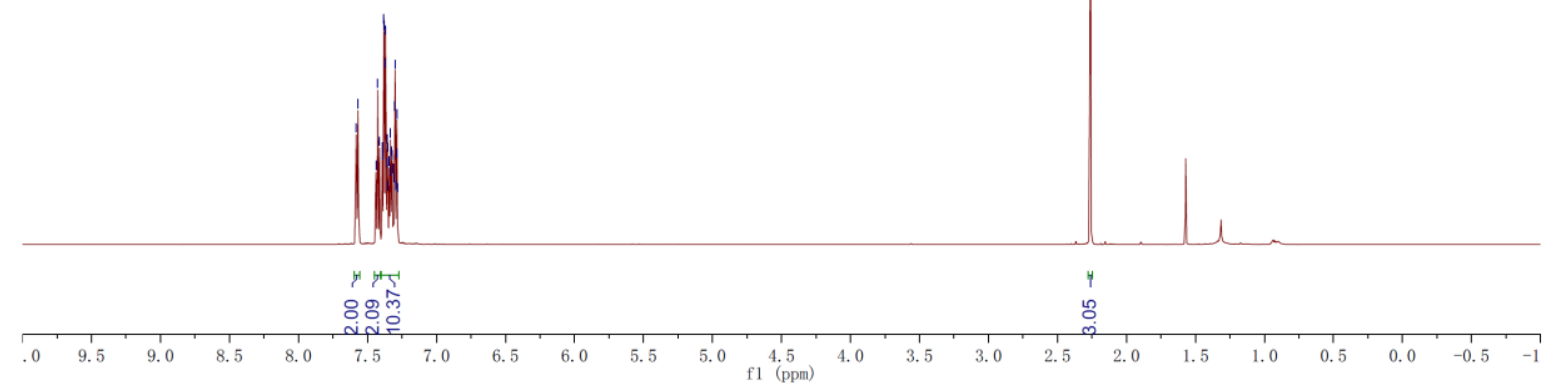

${ }^{13}$ C NMR (151 MHz, Chloroform- $d$ )

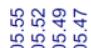

กู่ํํำ

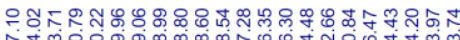

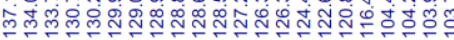

กิ่<smiles>Cc1ccccc1C(=C=C(c1ccccc1)C(F)(F)F)c1ccccc1</smiles>

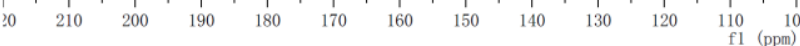


${ }^{19}$ F NMR (565 MHz, Chloroform- $d$ )

|

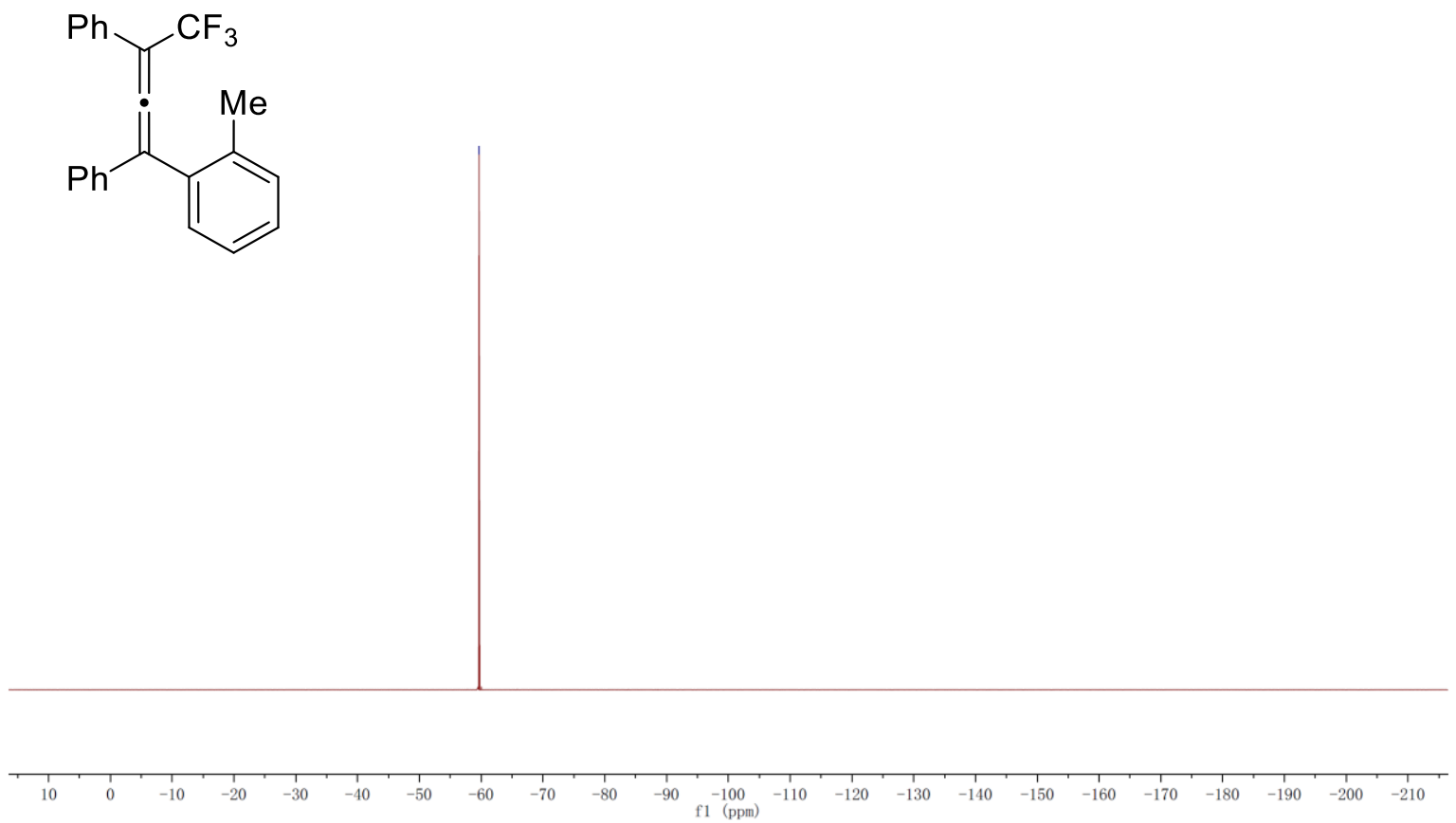


(4,4,4-Trifluoro-1-(2-fluorophenyl)buta-1,2-diene-1,3-diyl)dibenzene (12n)

${ }^{1}$ H NMR (600 MHz, Chloroform- $d$ )

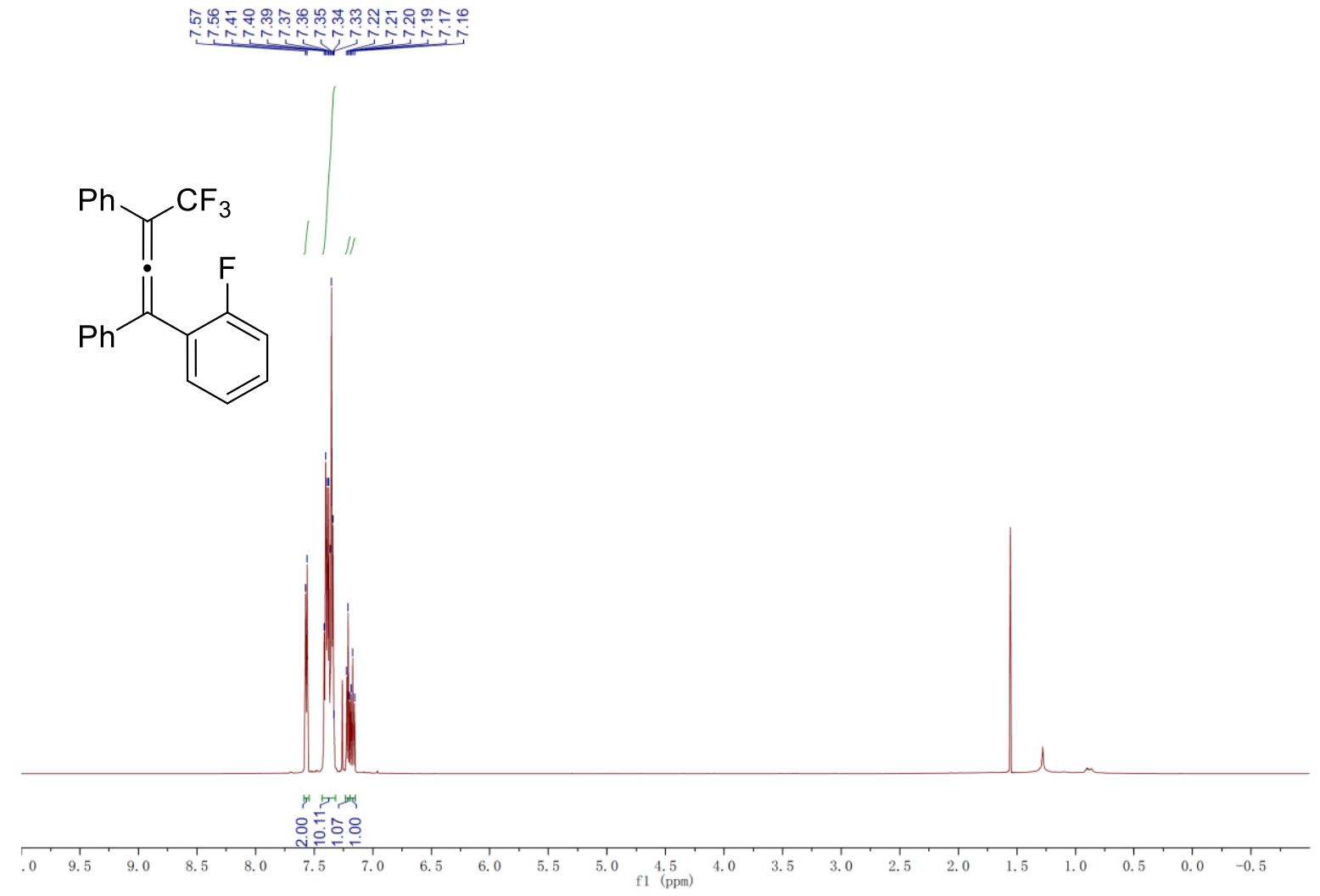

${ }^{13}$ C NMR (151 MHz, Chloroform- $d$ )
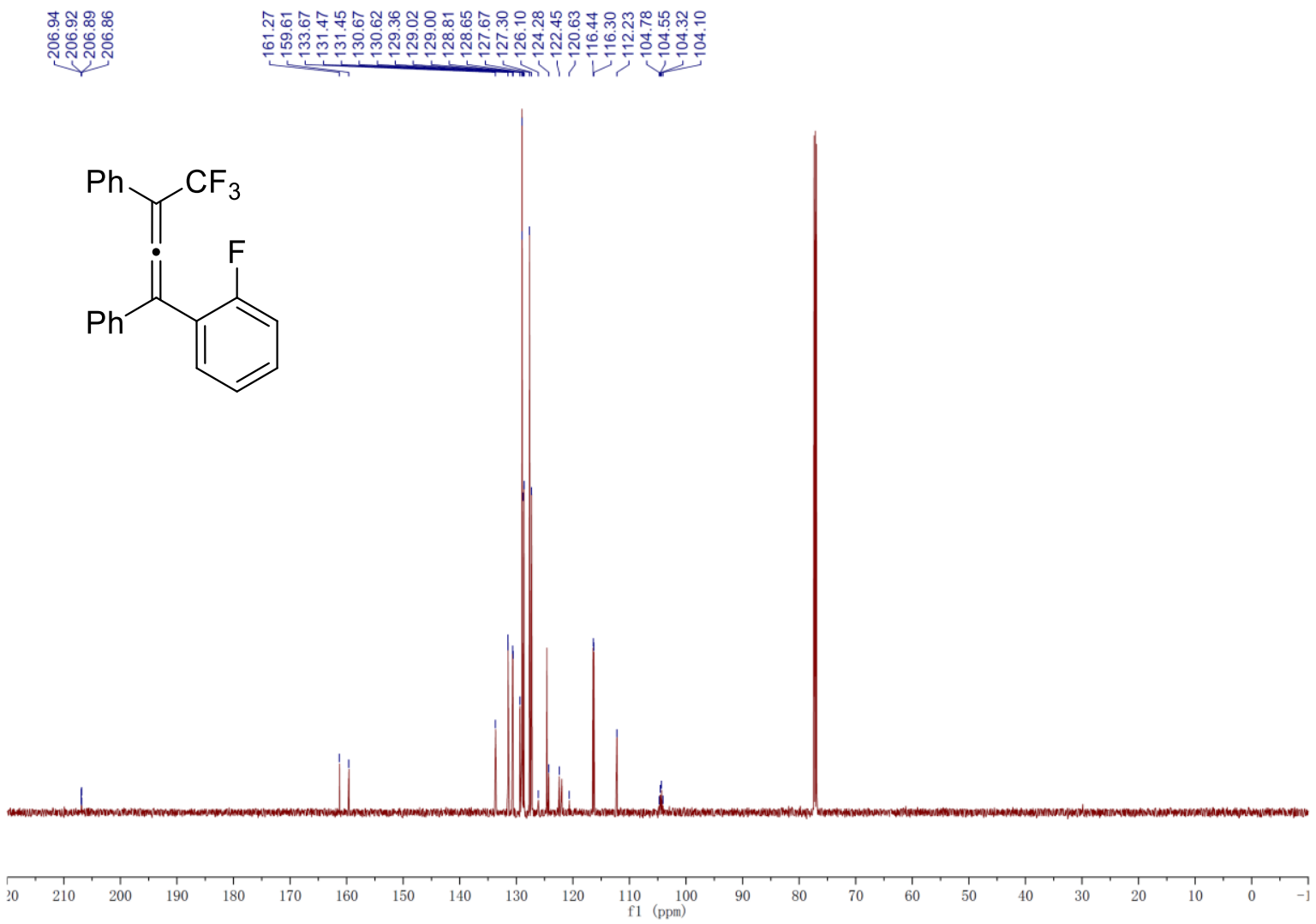
${ }^{19}$ F NMR (565 MHz, Chloroform- $d$ )

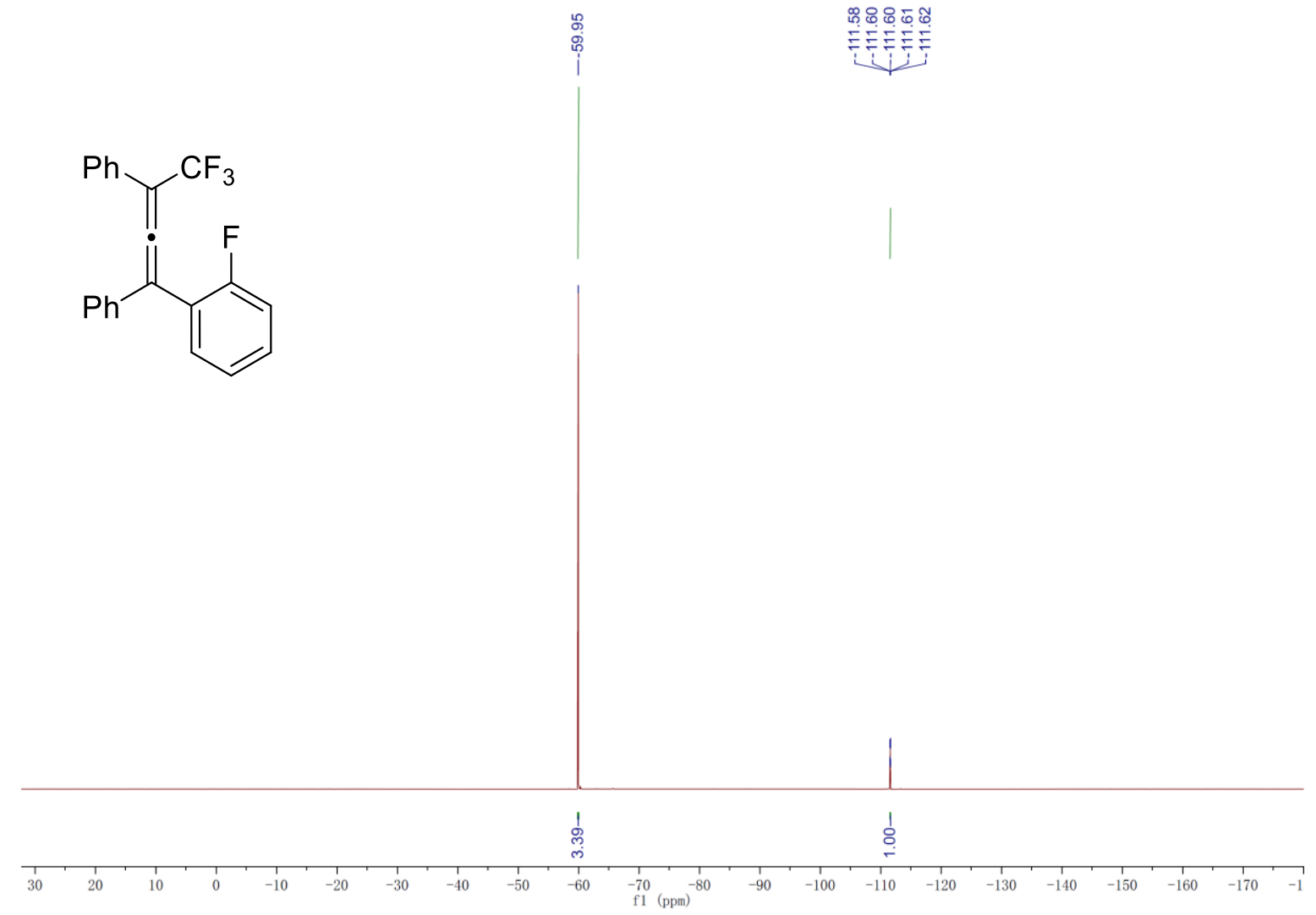


(1-(2-Chlorophenyl)-4,4,4-trifluorobuta-1,2-diene-1,3-diyl)dibenzene (12o)

${ }^{1}$ H NMR (600 MHz, Chloroform- $d$ )

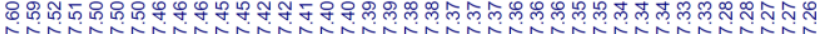

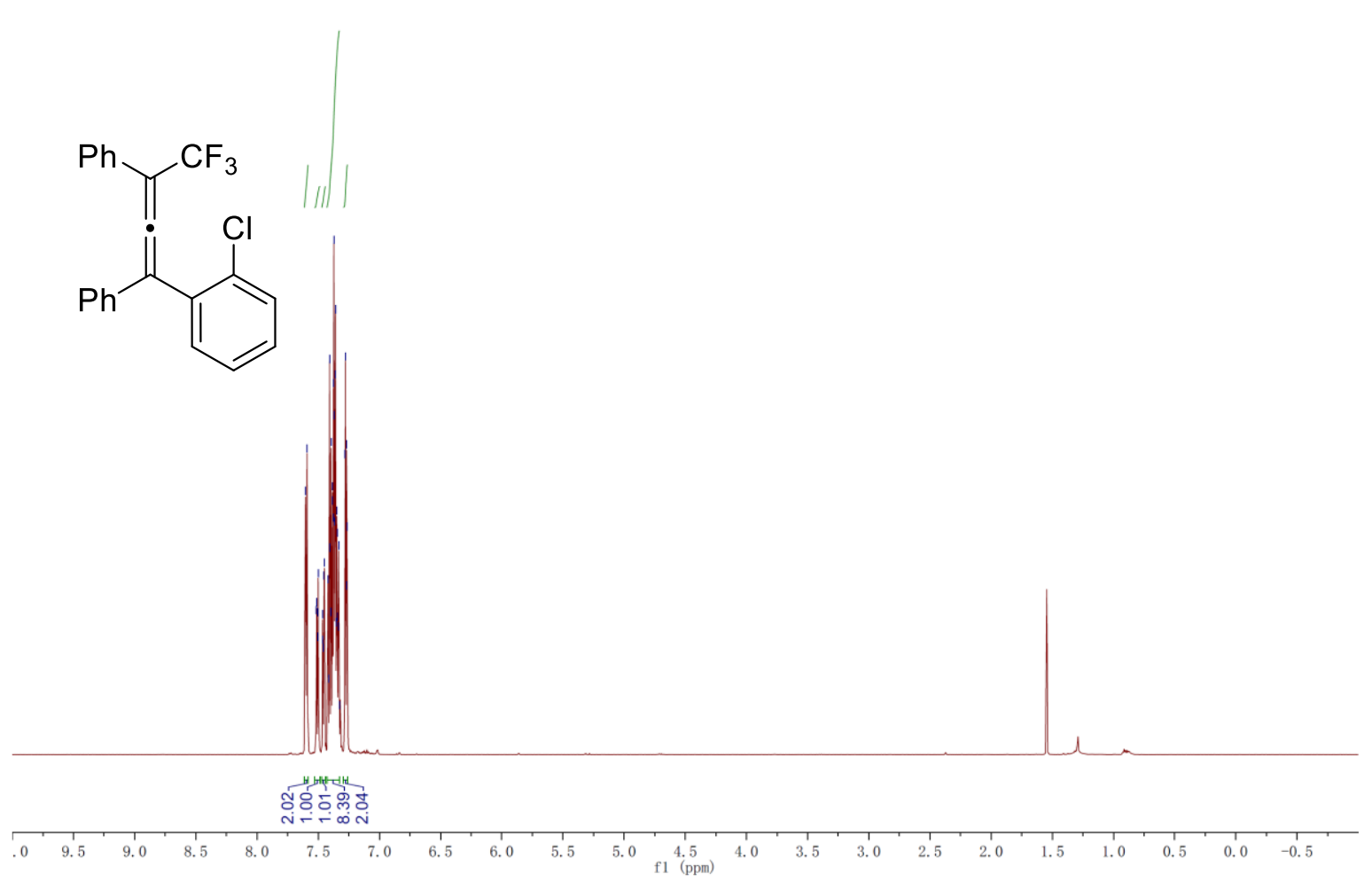

${ }^{13}$ C NMR (151 MHz, Chloroform-d)

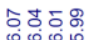

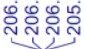

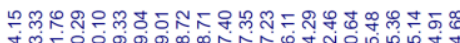

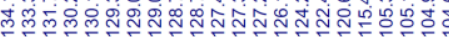

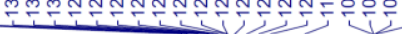<smiles>FC(F)(F)C(=C=C(c1ccccc1)c1ccccc1Cl)c1ccccc1</smiles>

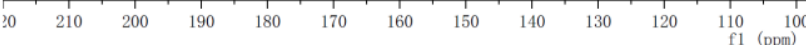


${ }^{19}$ F NMR (565 MHz, Chloroform- $d$ )

$$
\begin{aligned}
& \text { N } \\
& \text { i } \\
& \text { i }
\end{aligned}
$$

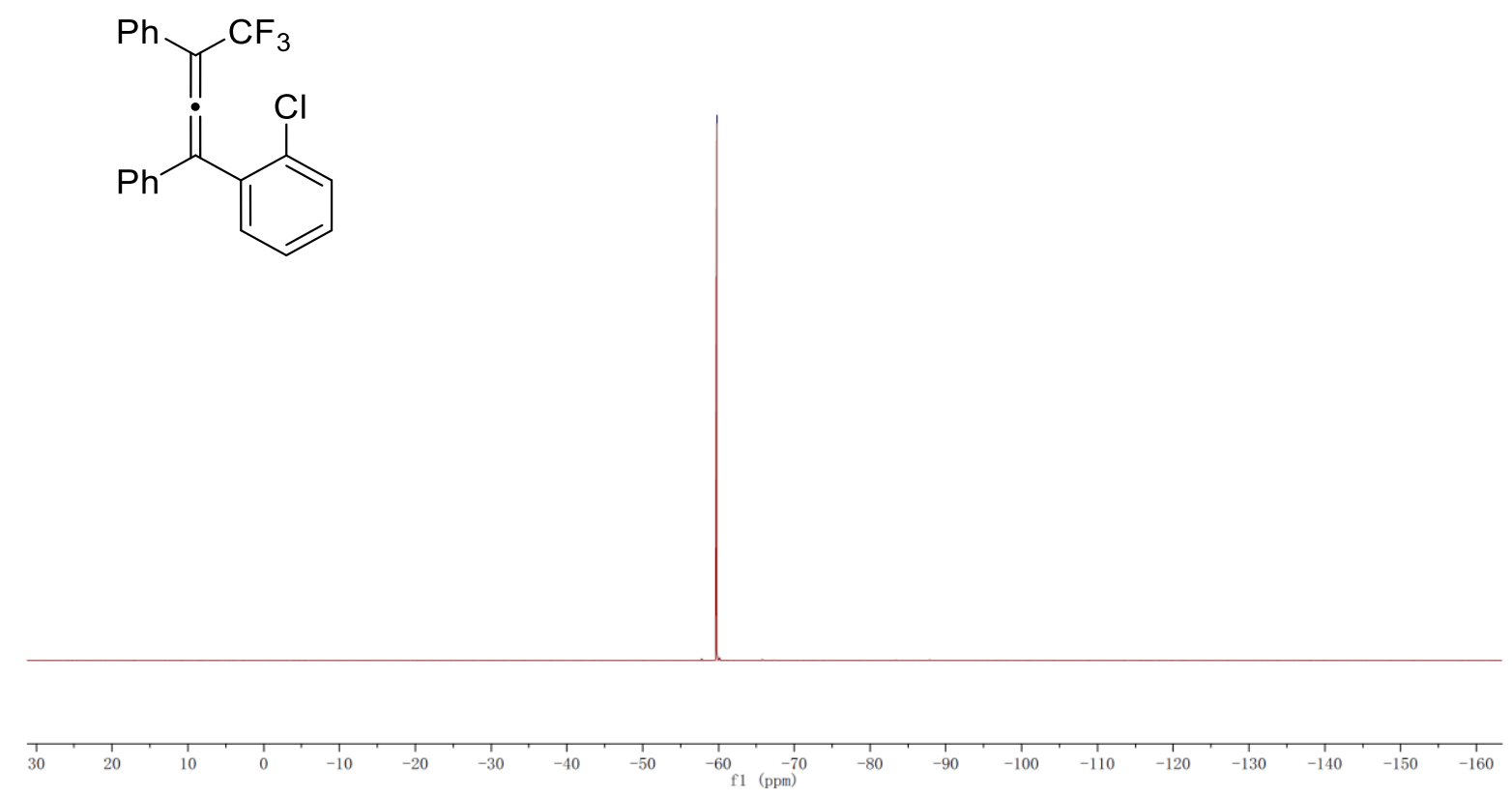


2-(4,4,4-Trifluoro-1,3-diphenylbuta-1,2-dien-1-yl)naphthalene (12p)

${ }^{1}$ H NMR (600 MHz, Chloroform- $d$ )

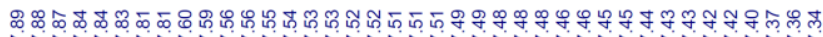

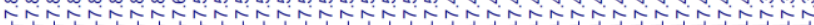<smiles>FC(F)(F)C(=C=C(c1ccccc1)c1ccc2ccccc2c1)c1ccccc1</smiles>

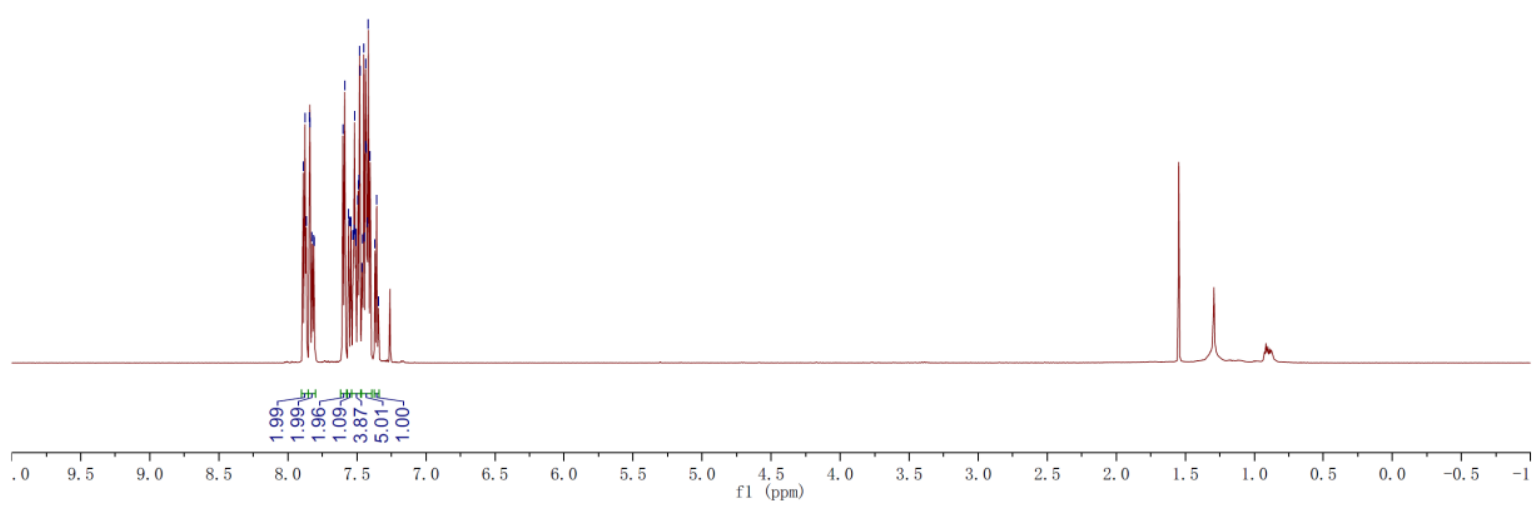

${ }^{13}$ C NMR (151 MHz, Chloroform- $d$ )

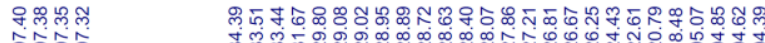

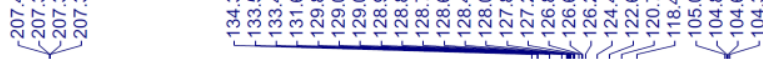

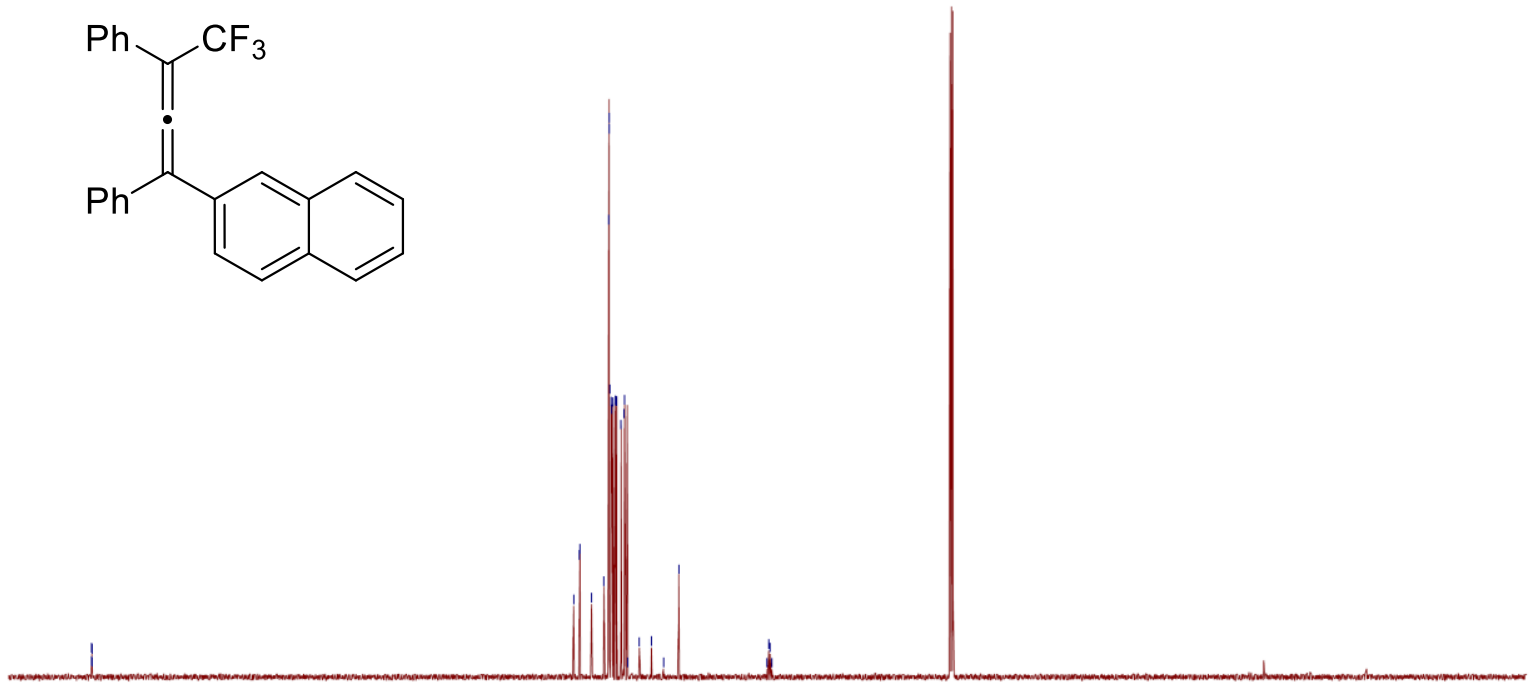

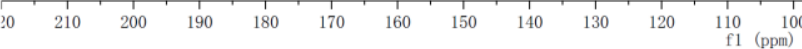


${ }^{19}$ F NMR (565 MHz, Chloroform- $d$ )<smiles>FC(F)(F)C(=C=C(c1ccccc1)c1ccc2ccccc2c1)c1ccccc1</smiles>

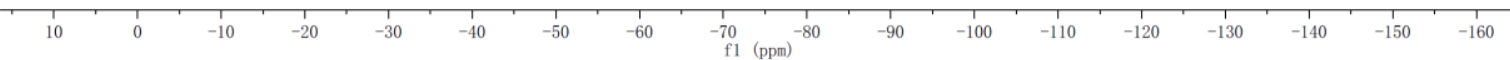


1-(4,4,4-Trifluoro-1,3-diphenylbuta-1,2-dien-1-yl)naphthalene (12q)

${ }^{1}$ H NMR (600 MHz, Chloroform- $d$ )

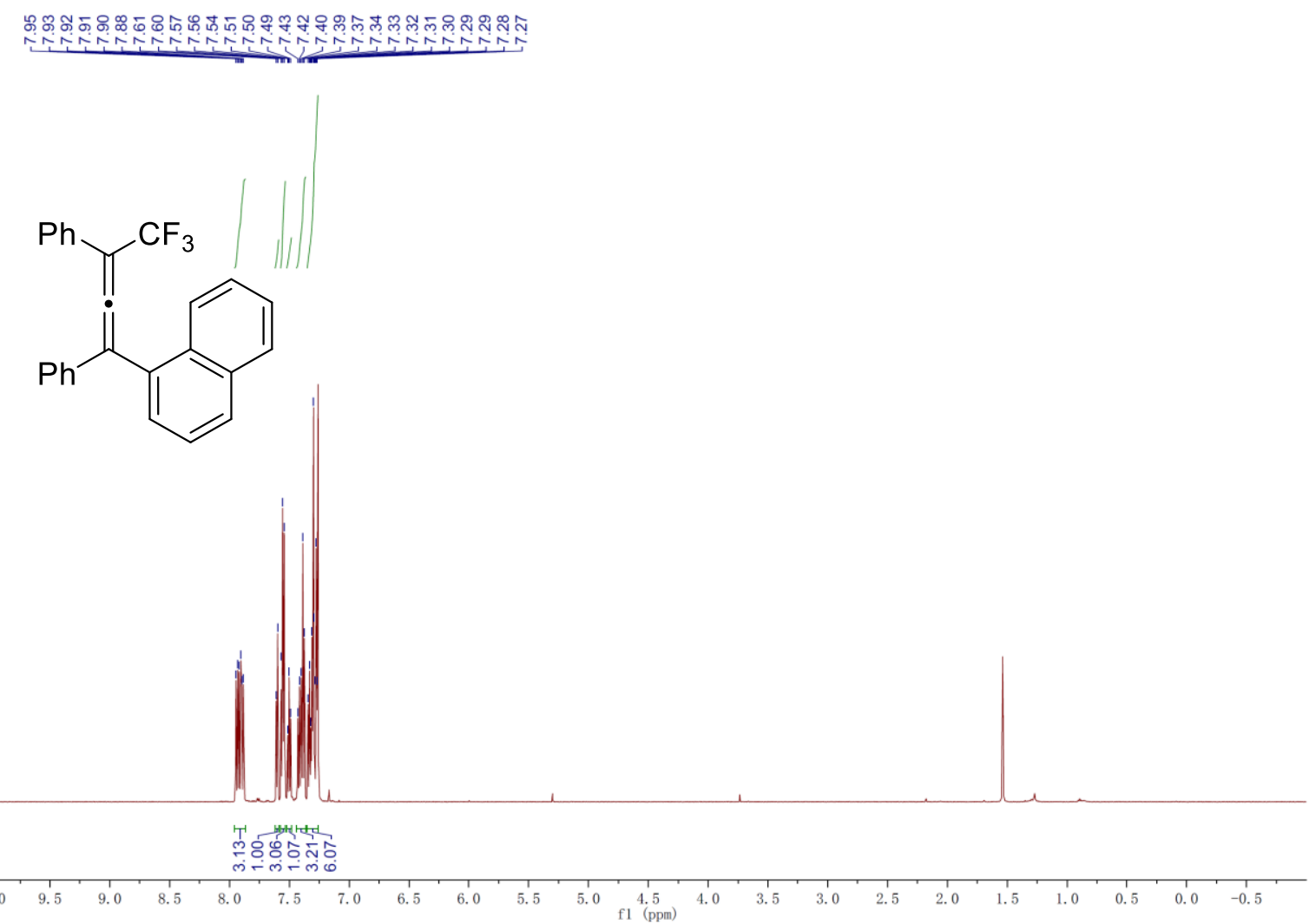

${ }^{13}$ C NMR (151 MHz, Chloroform-d)

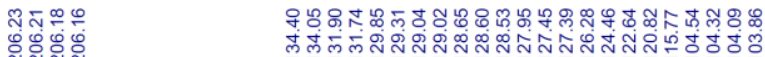

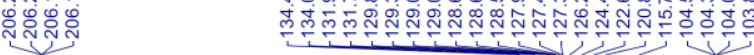

(c)

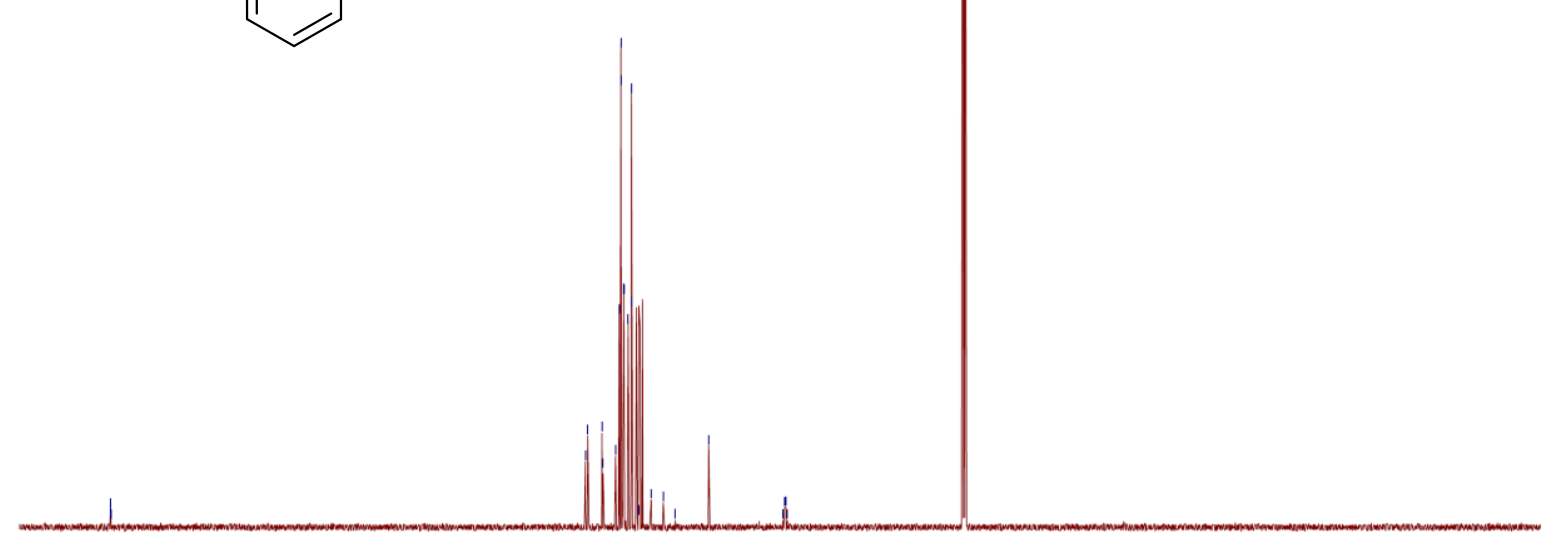

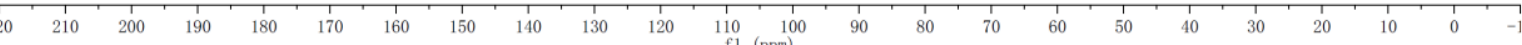


${ }^{19}$ F NMR (565 MHz, Chloroform- $d$ )

$$
\text { ํㅗㅇ }
$$<smiles>FC(F)(F)C(=NC(c1ccccc1)c1ccccc1)c1ccccc1</smiles>

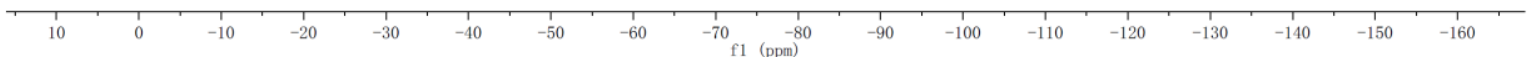


2-(4,4,4-Trifluoro-1,3-diphenylbuta-1,2-dien-1-yl)thiophene (12r)

${ }^{1}$ H NMR (600 MHz, Chloroform- $d$ )

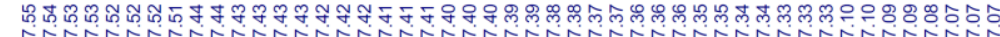

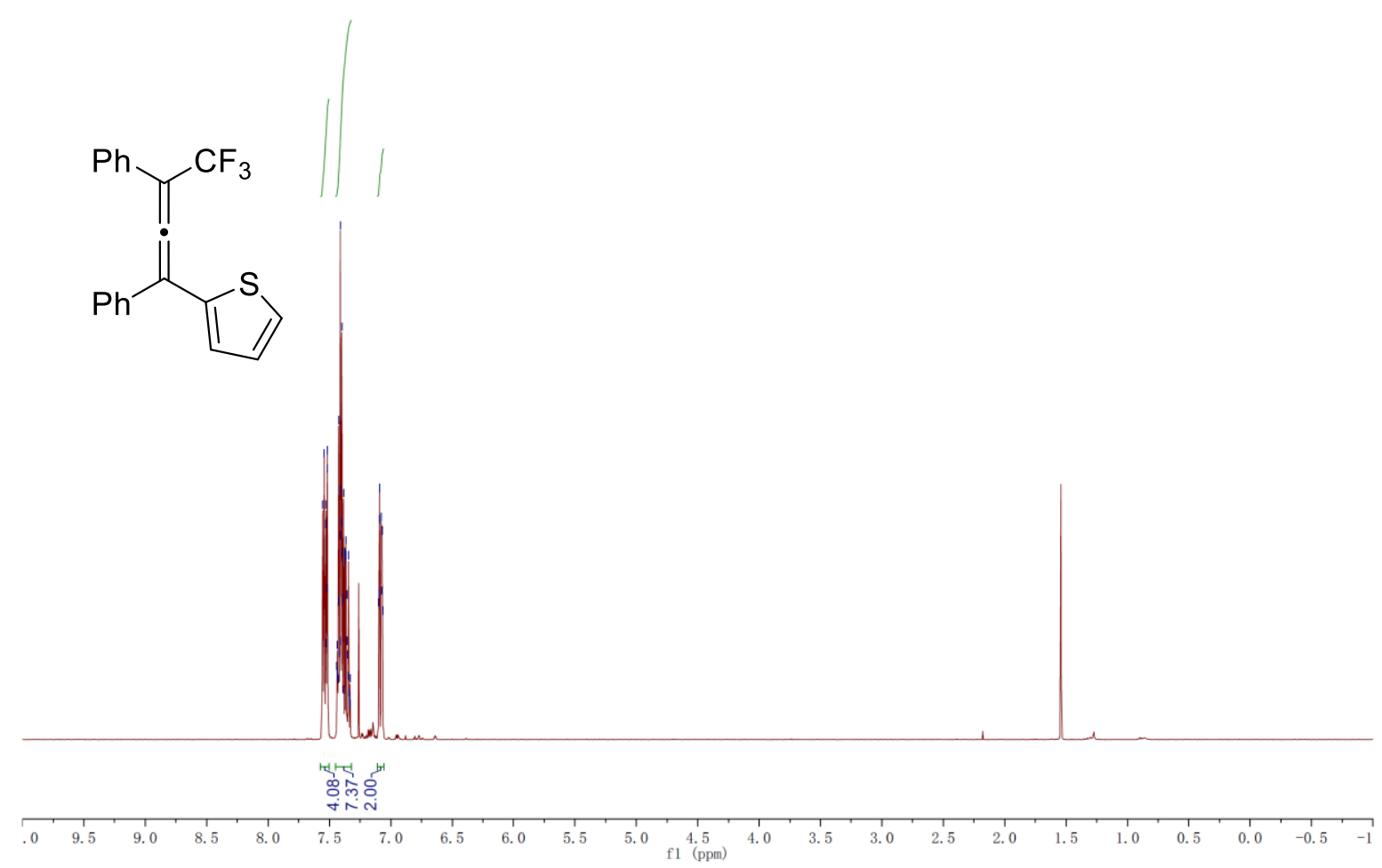

${ }^{13}$ C NMR (151 MHz, Chloroform- $d$ )

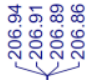

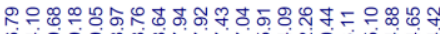

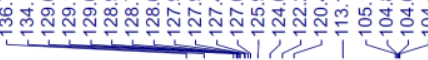

Nin<smiles>FC(F)(F)C(=C=C(c1ccccc1)c1cccs1)c1ccccc1</smiles>

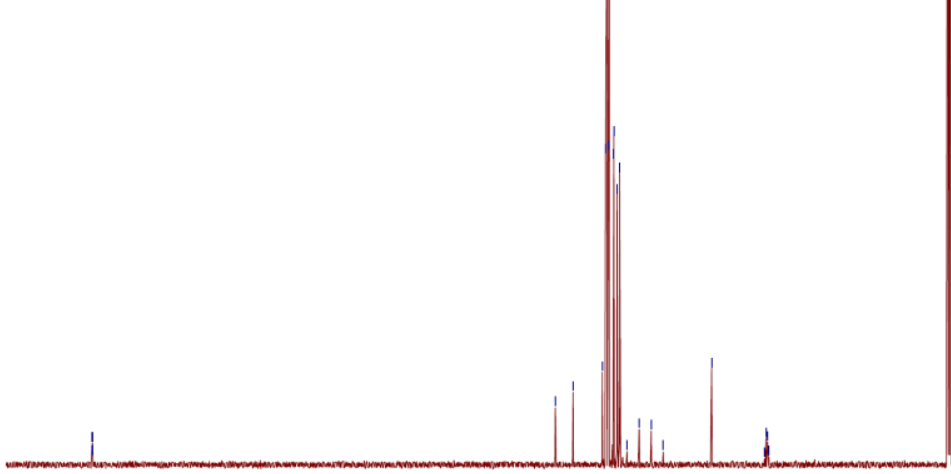

$\begin{array}{llllllllllll}20 & 210 & 200 & 190 & 180 & 170 & 160 & 150 & 140 & 130 & 120 & 110 \\ \mathrm{f} 1(\mathrm{ppm}) & 100\end{array}$ 
${ }^{19}$ F NMR (565 MHz, Chloroform- $d$ )

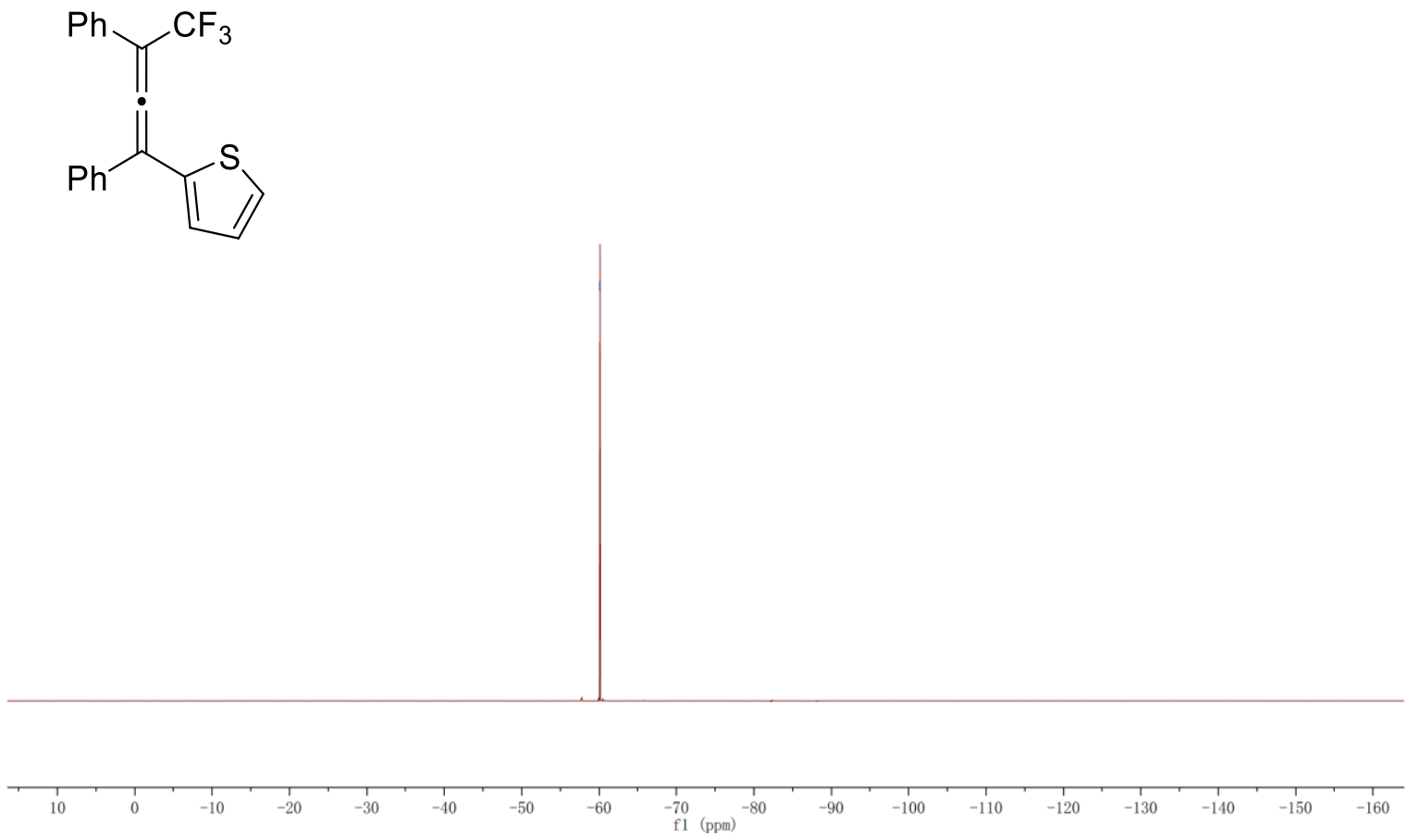


5-(3,3,3-Trifluoro-2-phenylprop-1-en-1-ylidene)-10,11-dihydro-5H-dibenzo[a,d][7]annulene (12s) ${ }^{1}$ H NMR (600 MHz, Chloroform- $d$ )

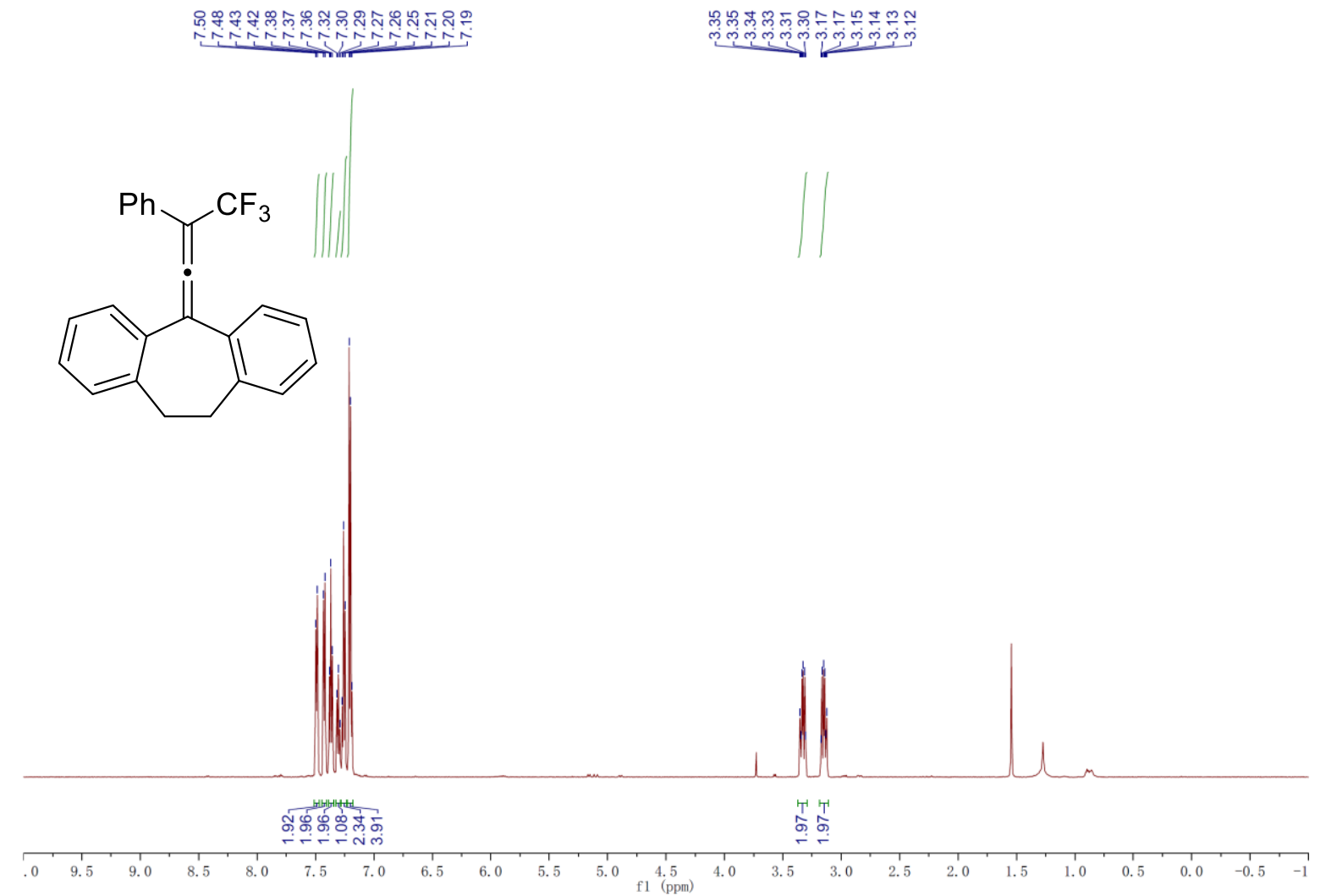

${ }^{13}$ C NMR (151 MHz, Chloroform-d)

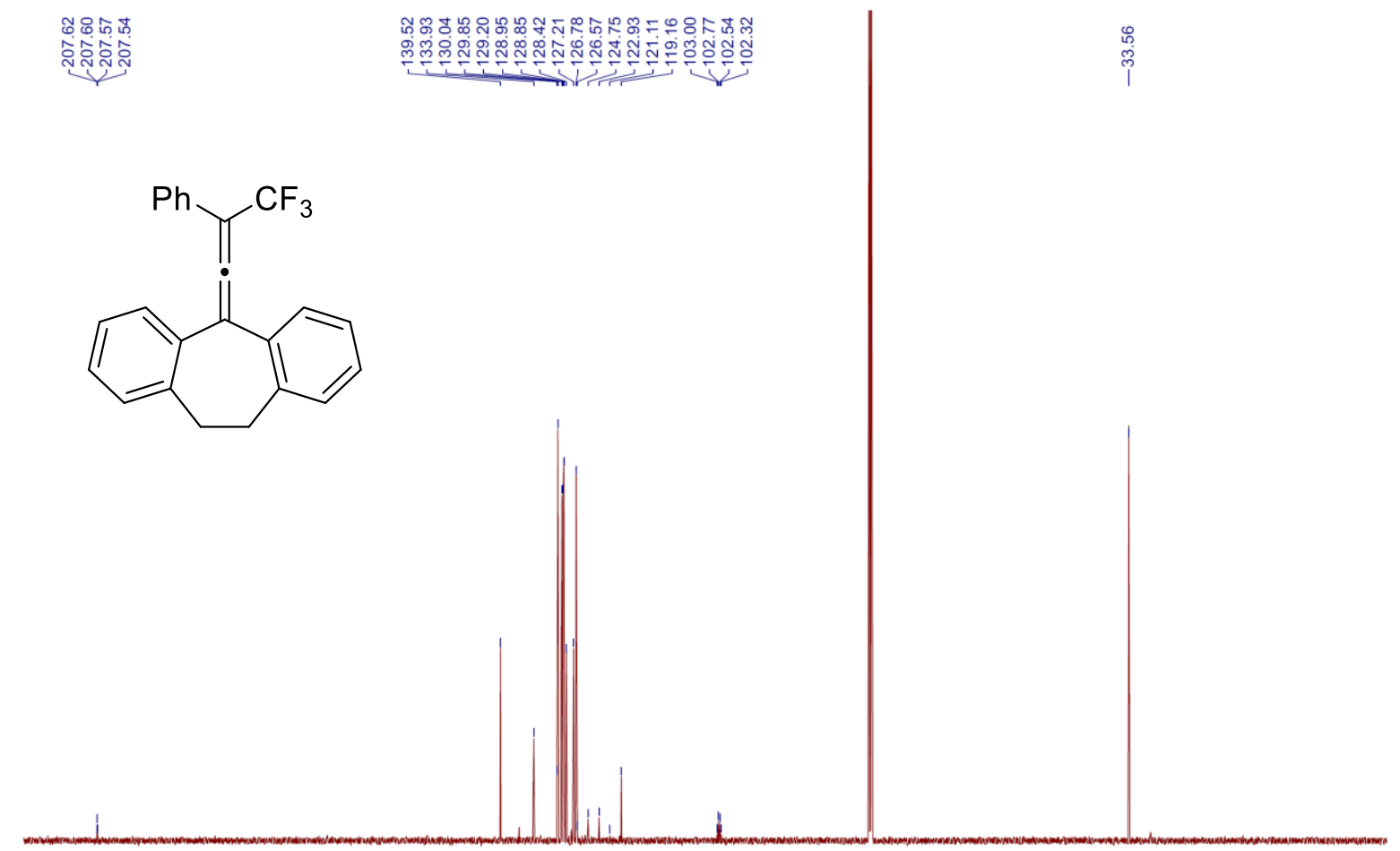


${ }^{19}$ F NMR (565 MHz, Chloroform- $d$ )

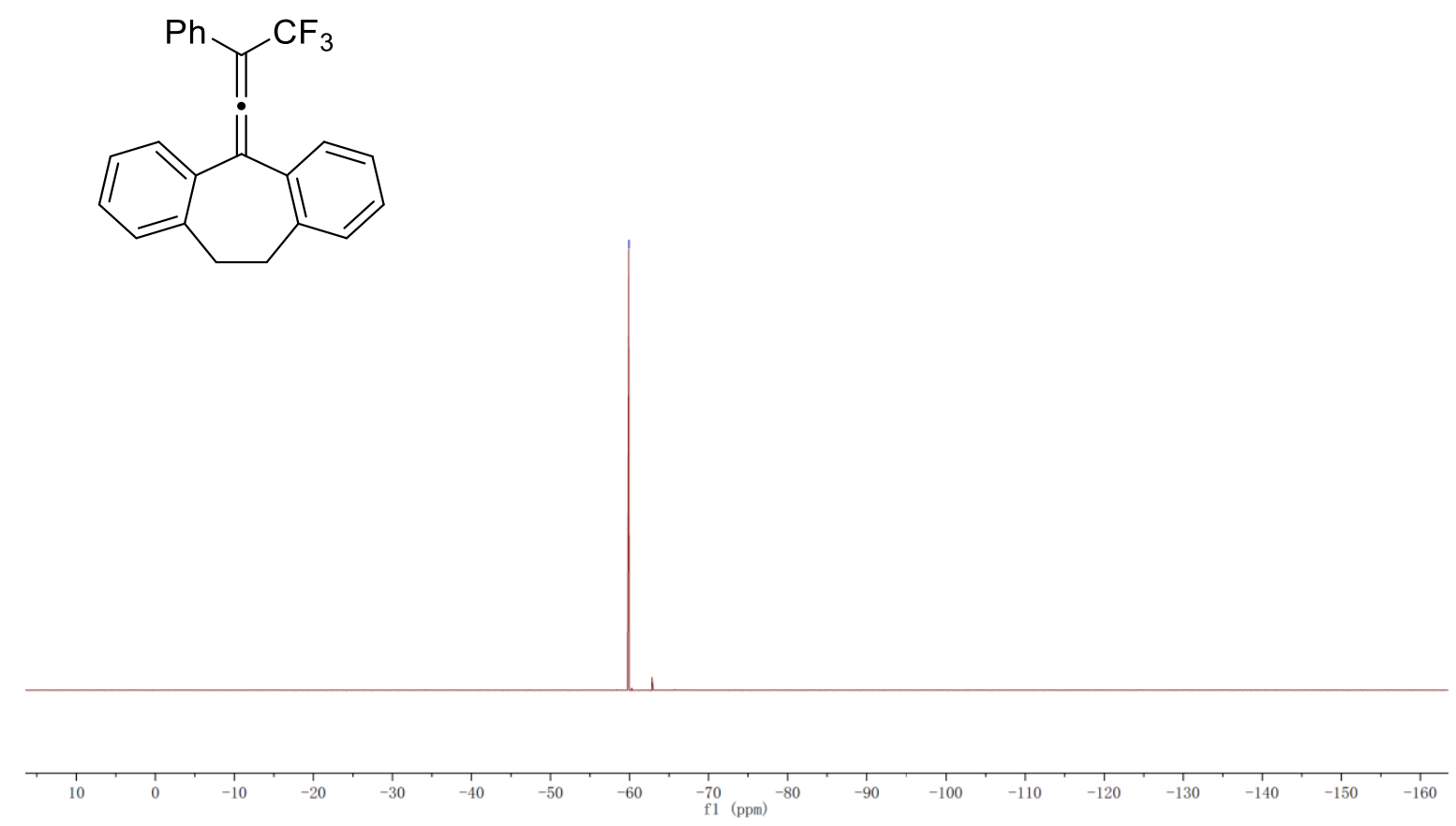


5-(3,3,3-Trifluoro-2-phenylprop-1-en-1-ylidene)-5H-dibenzo[ $a, d][7]$ annulene (12t)

${ }^{1}$ H NMR (600 MHz, Chloroform- $d$ )

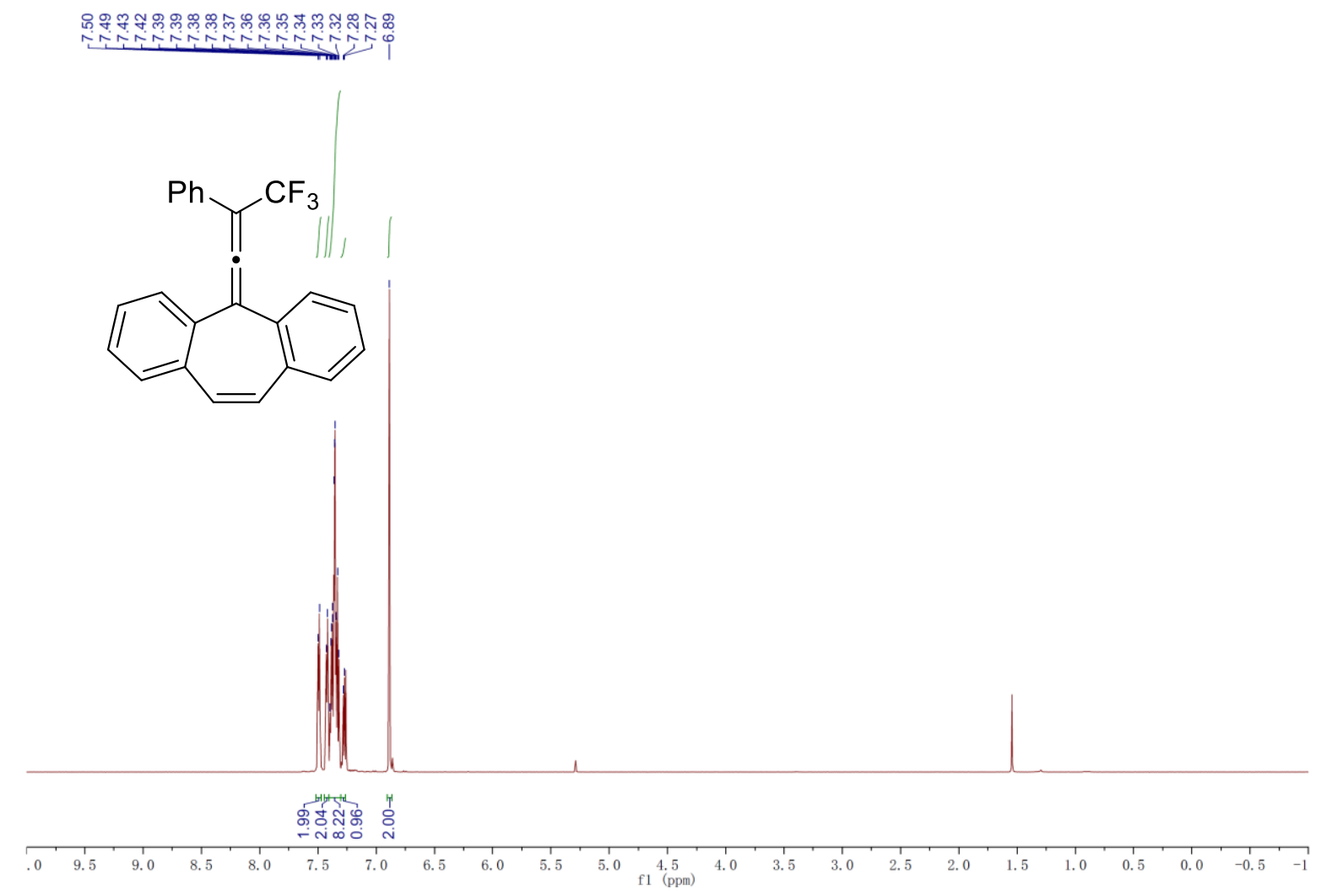

${ }^{13}$ C NMR (151 MHz, Chloroform- $d$ )

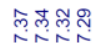
จิํํํํํ

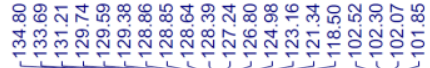

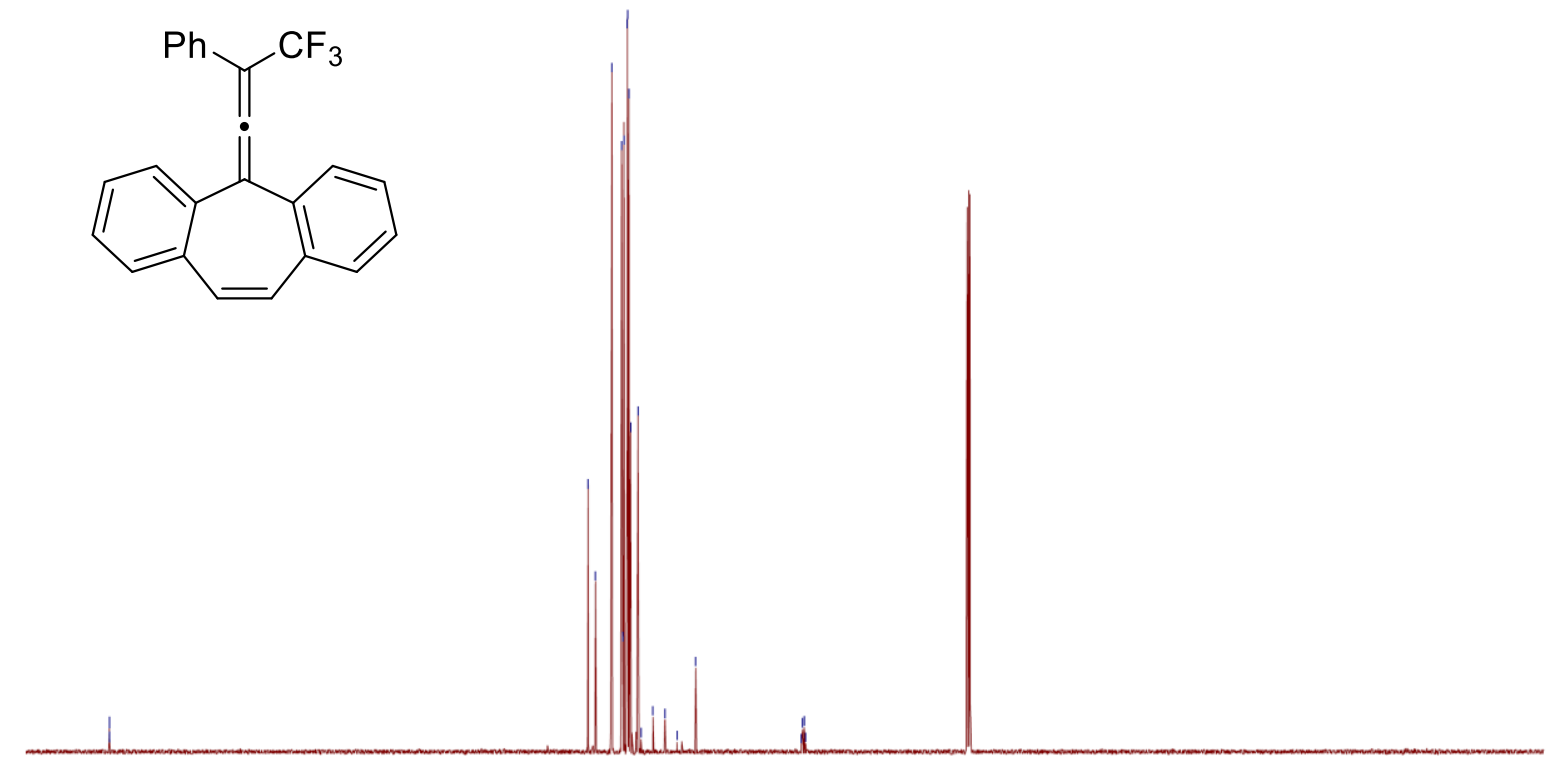

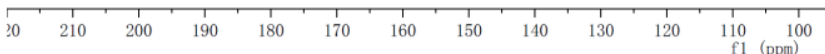


${ }^{19}$ F NMR (565 MHz, Chloroform- $d$ )

$$
\text { | }
$$<smiles>FC(F)(F)C(=C=C1c2ccccc2C=Cc2ccccc21)c1ccccc1</smiles>

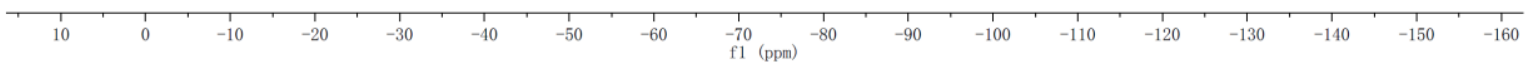


(3-(4-Chlorophenyl)-4,4,4-trifluorobuta-1,2-diene-1,1-diyl)dibenzene (12u)

${ }^{1}$ H NMR (600 MHz, Chloroform- $d$ )

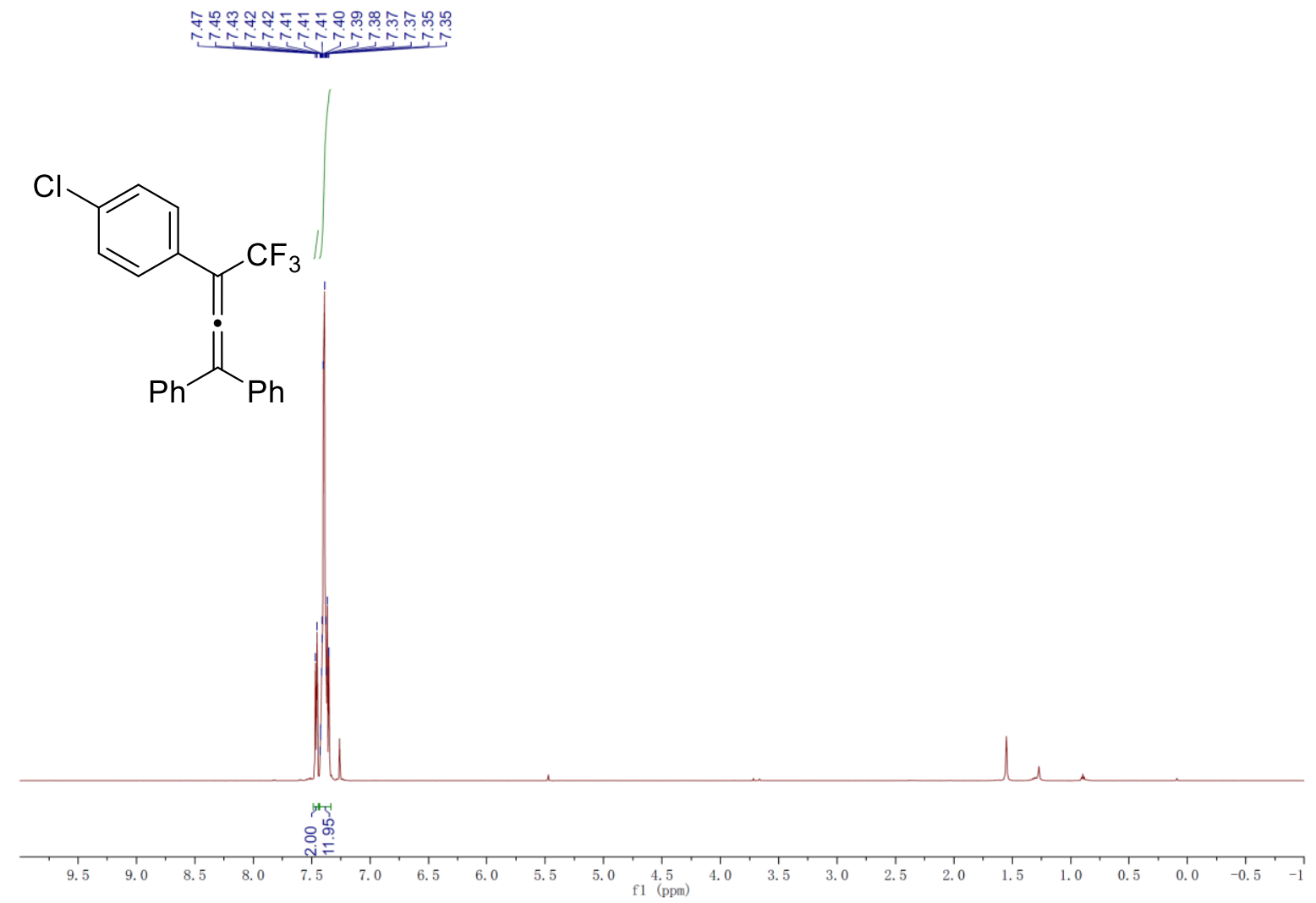

${ }^{13}$ C NMR (151 MHz, Chloroform- $\left.d\right)$

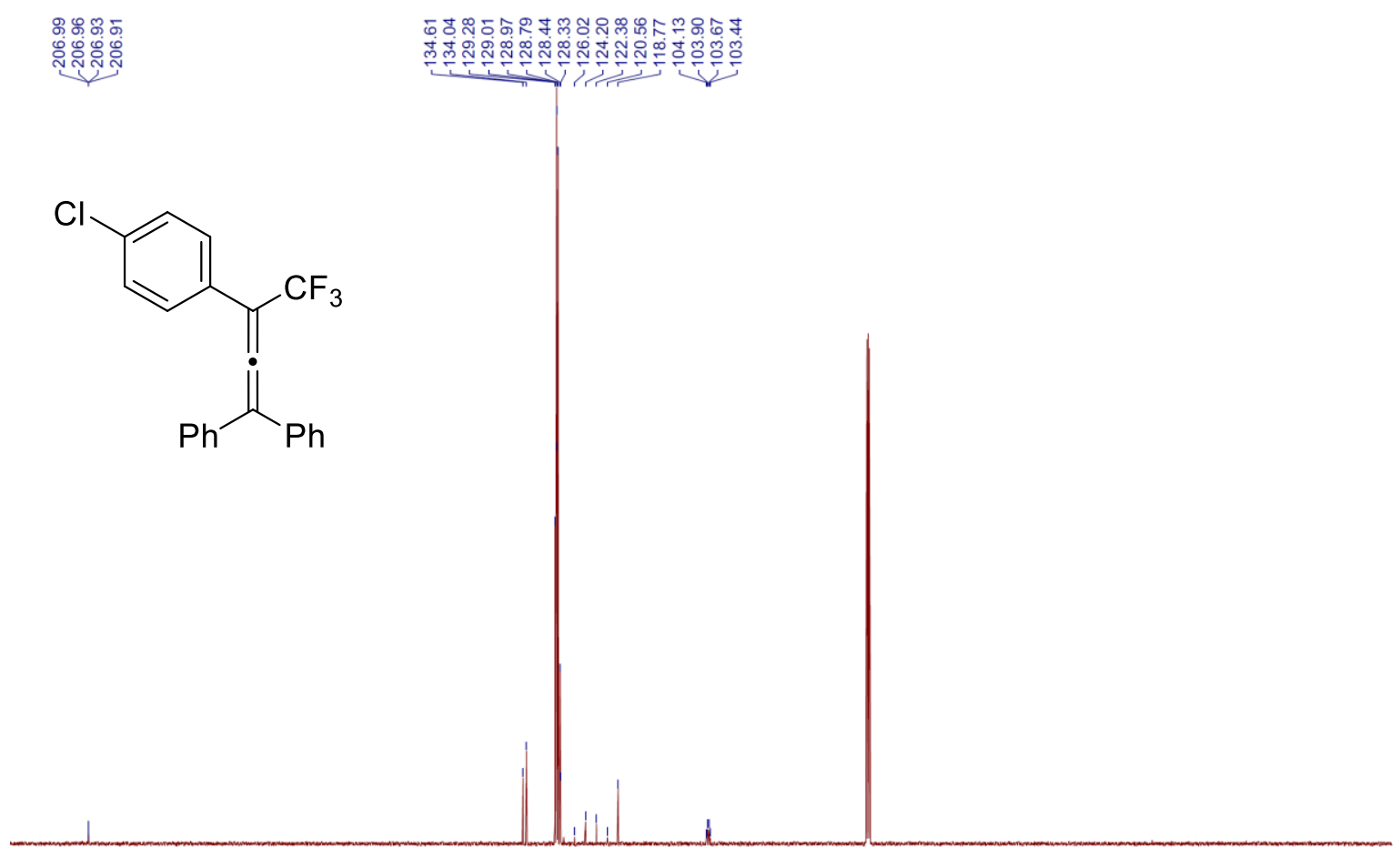

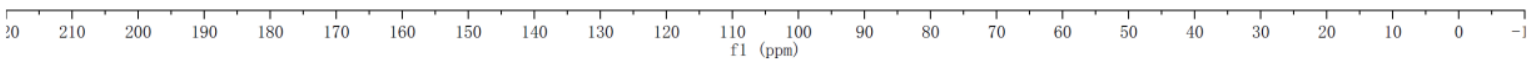


${ }^{19}$ F NMR (565 MHz, Chloroform- $d$ )

$$
\text { 웅 }
$$<smiles>FC(F)(F)C(=C=C(c1ccccc1)c1ccccc1)c1ccc(Cl)cc1</smiles>

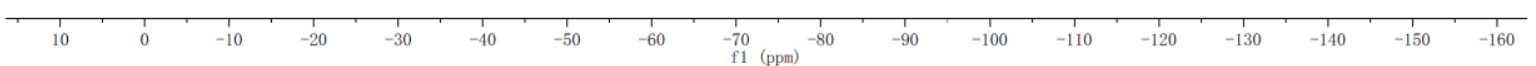


(4,4,4-Trifluoro-3-( $p$-tolyl)buta-1,2-diene-1,1-diyl)dibenzene (12v)

${ }^{1}$ H NMR (600 MHz, Chloroform- $d$ )

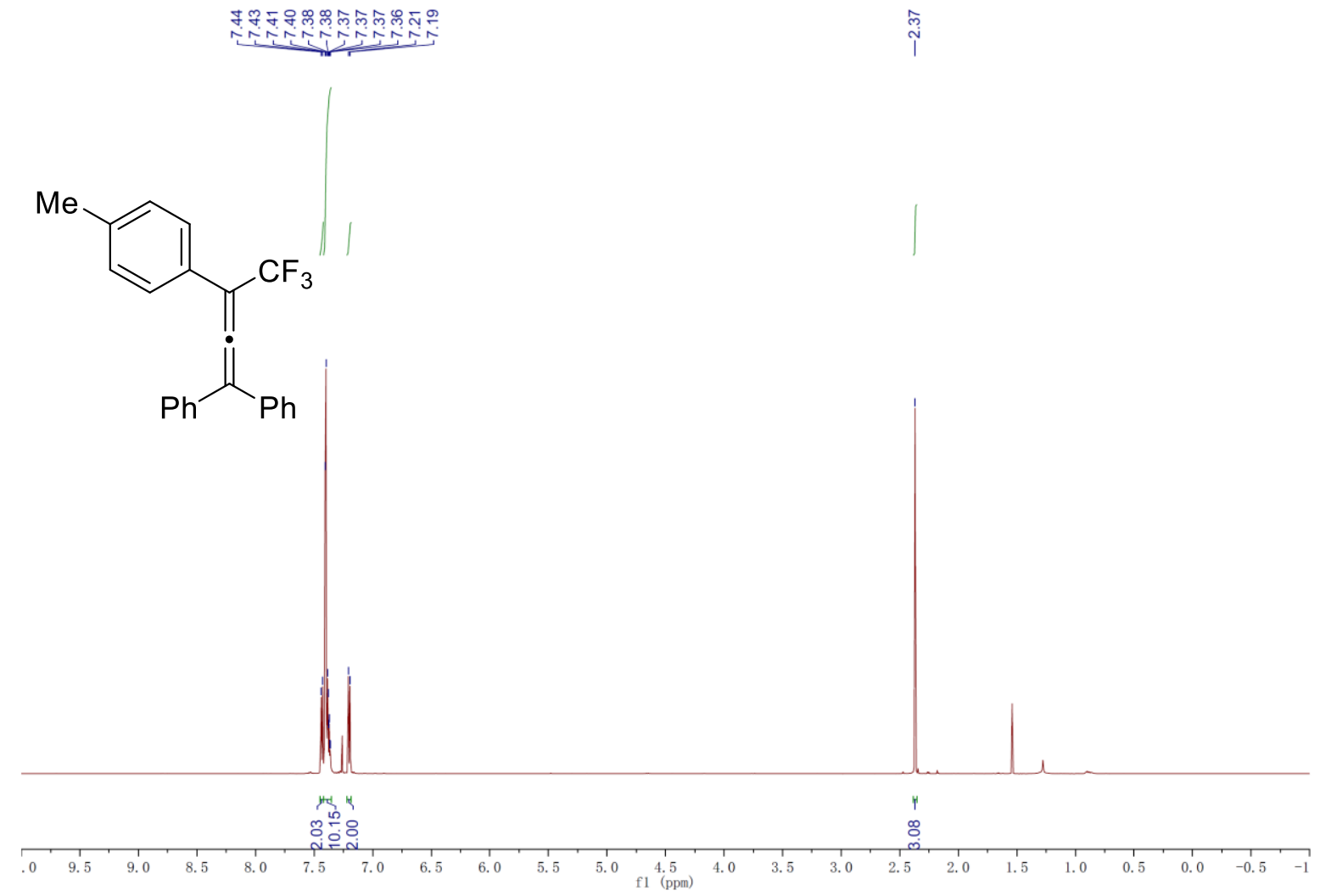

${ }^{13}$ C NMR (151 MHz, Chloroform- $d$ )

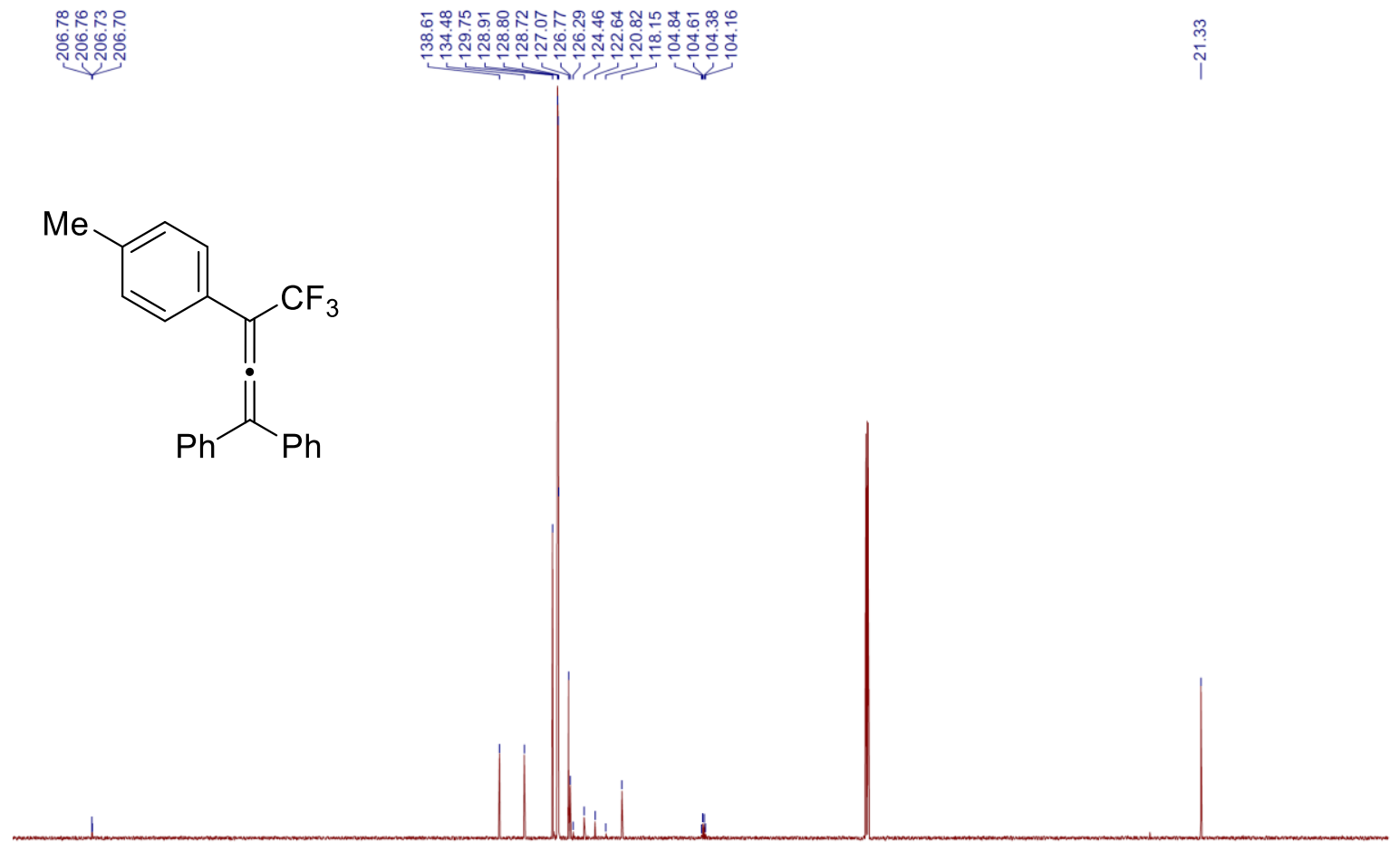

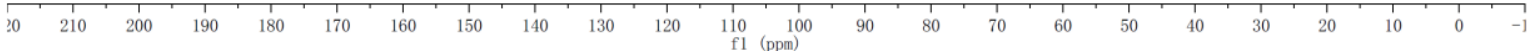


${ }^{19}$ F NMR (565 MHz, Chloroform- $d$ )

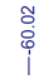<smiles>Cc1ccc(C(=C=C(c2ccccc2)c2ccccc2)C(F)(F)F)cc1</smiles>

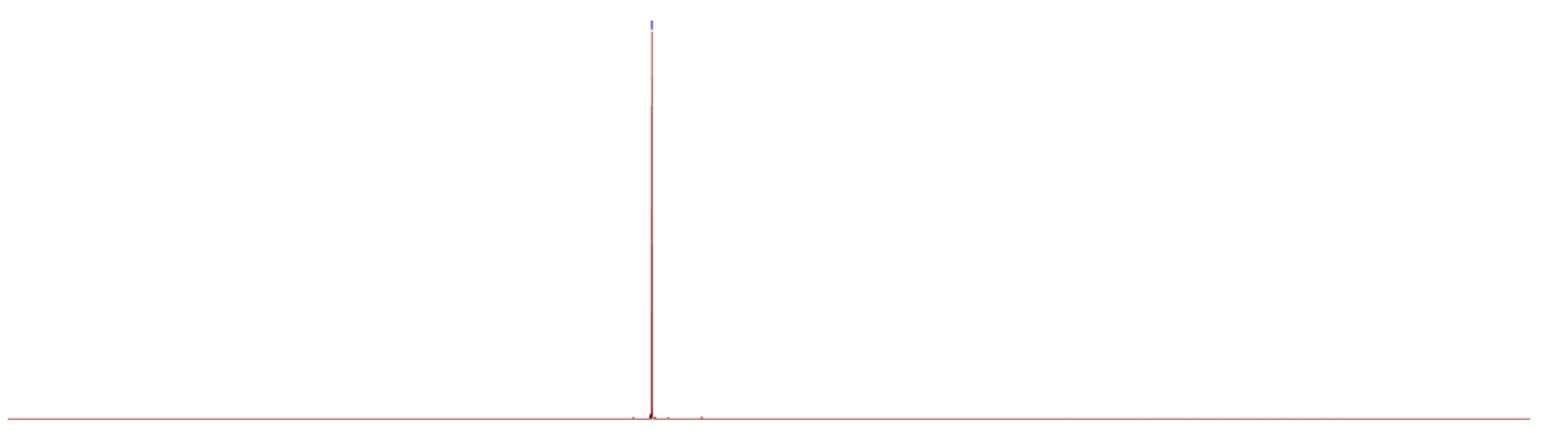

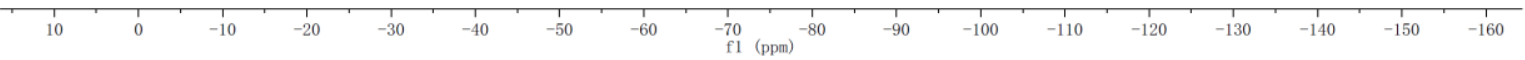


(4,4,4-Trifluoro-3-(4-fluorophenyl)buta-1,2-diene-1,1-diyl)dibenzene (12w)

${ }^{1}$ H NMR (600 MHz, Chloroform- $d$ )

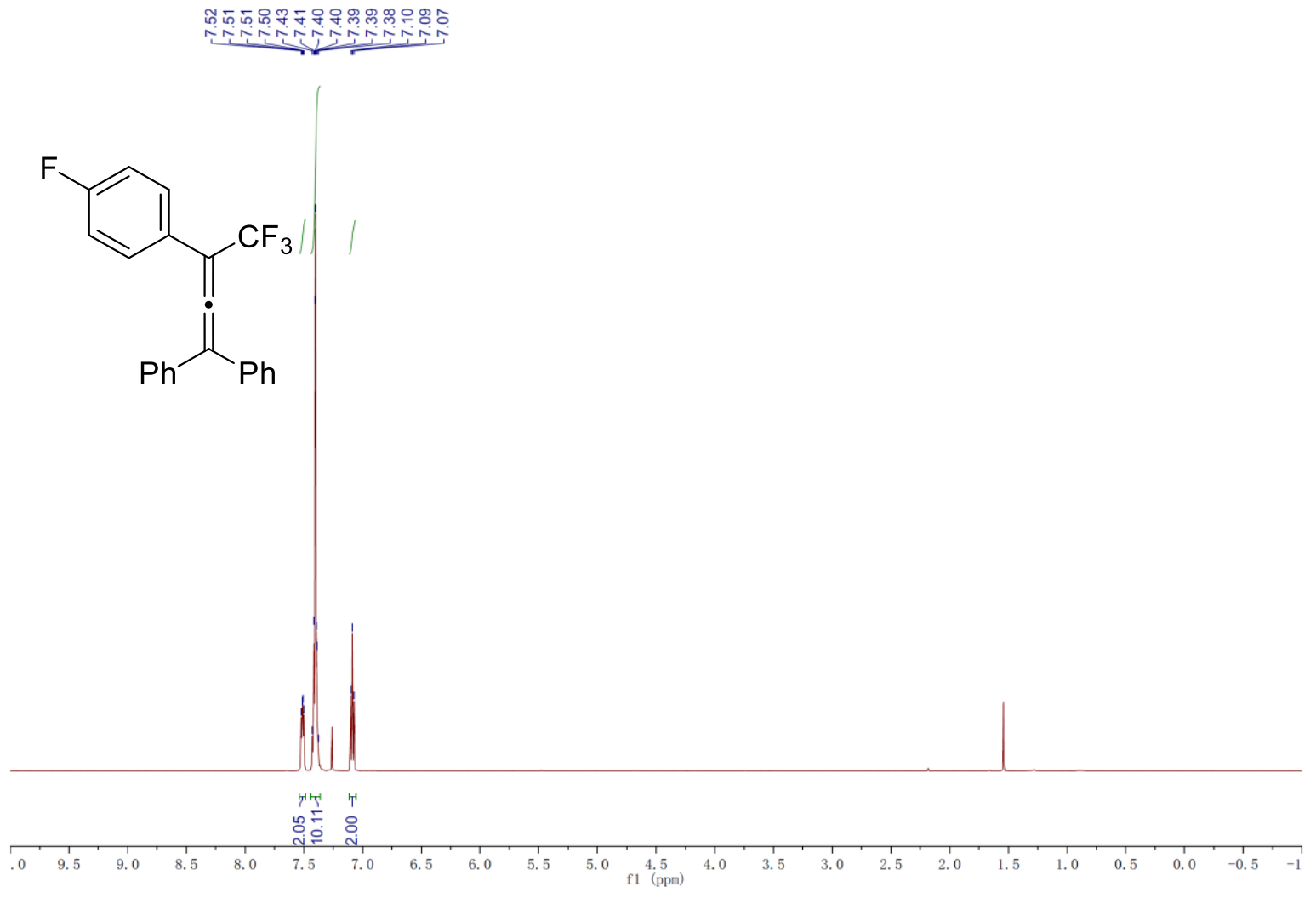

${ }^{13}$ C NMR (151 MHz, Chloroform-d)

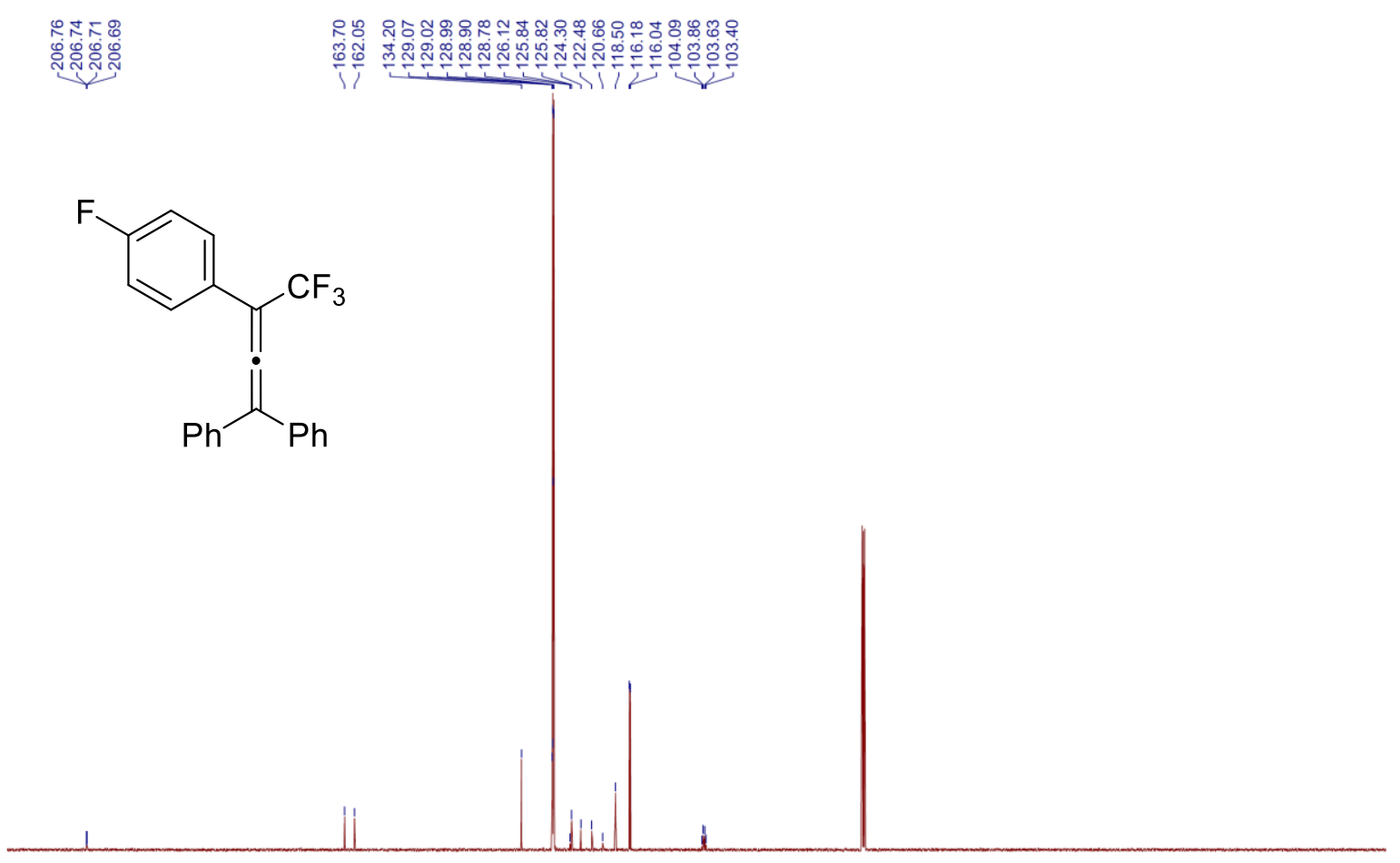

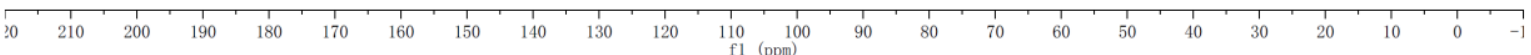


${ }^{19}$ F NMR (565 MHz, Chloroform- $d$ )

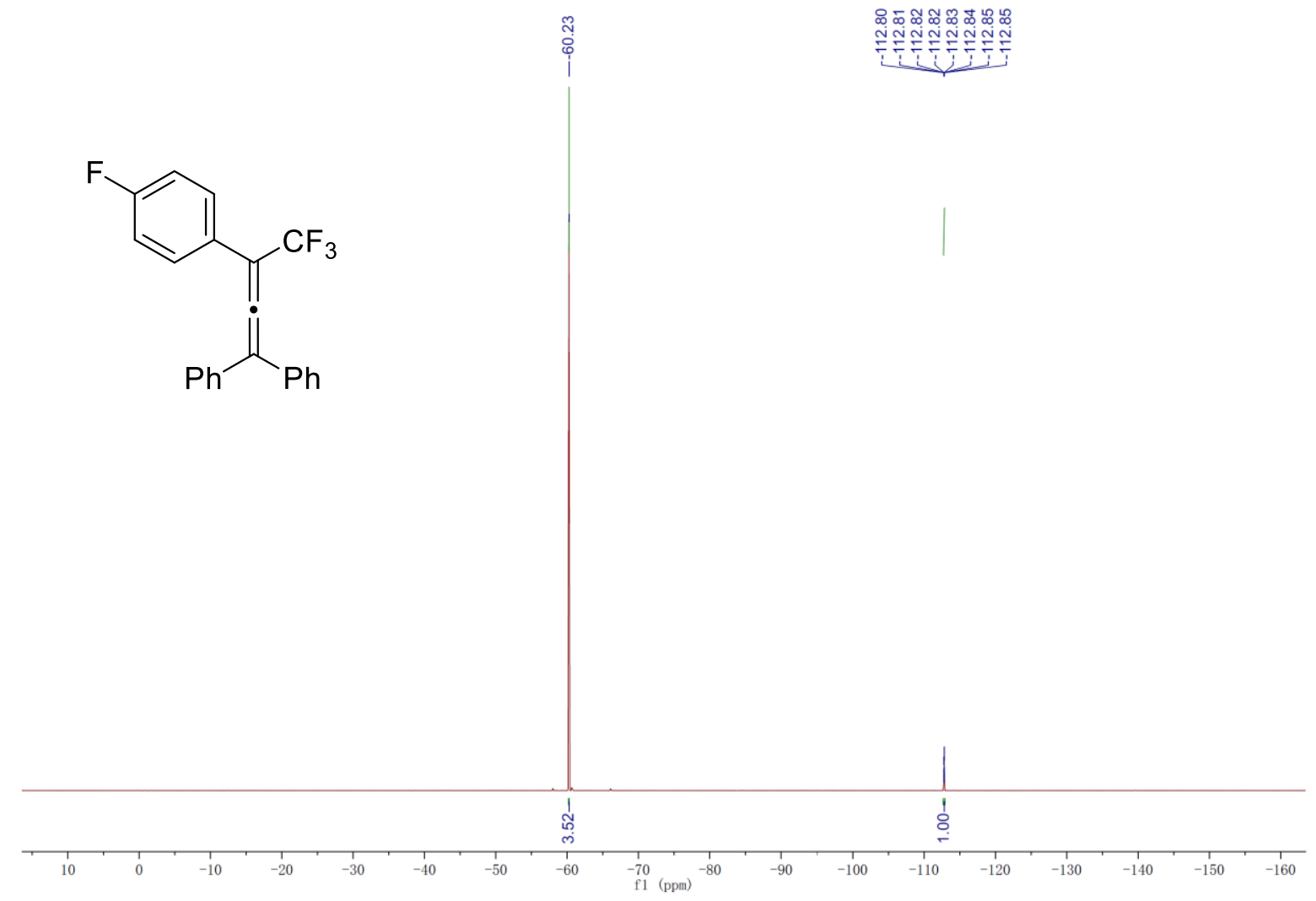


6-Methyl-1-phenyl-3-(p-tolyl)-1-(trifluoromethyl)-1H-indene (16)

${ }^{1}$ H NMR (400 MHz, Chloroform- $d$ )

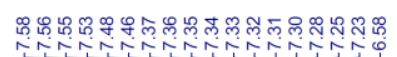

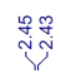
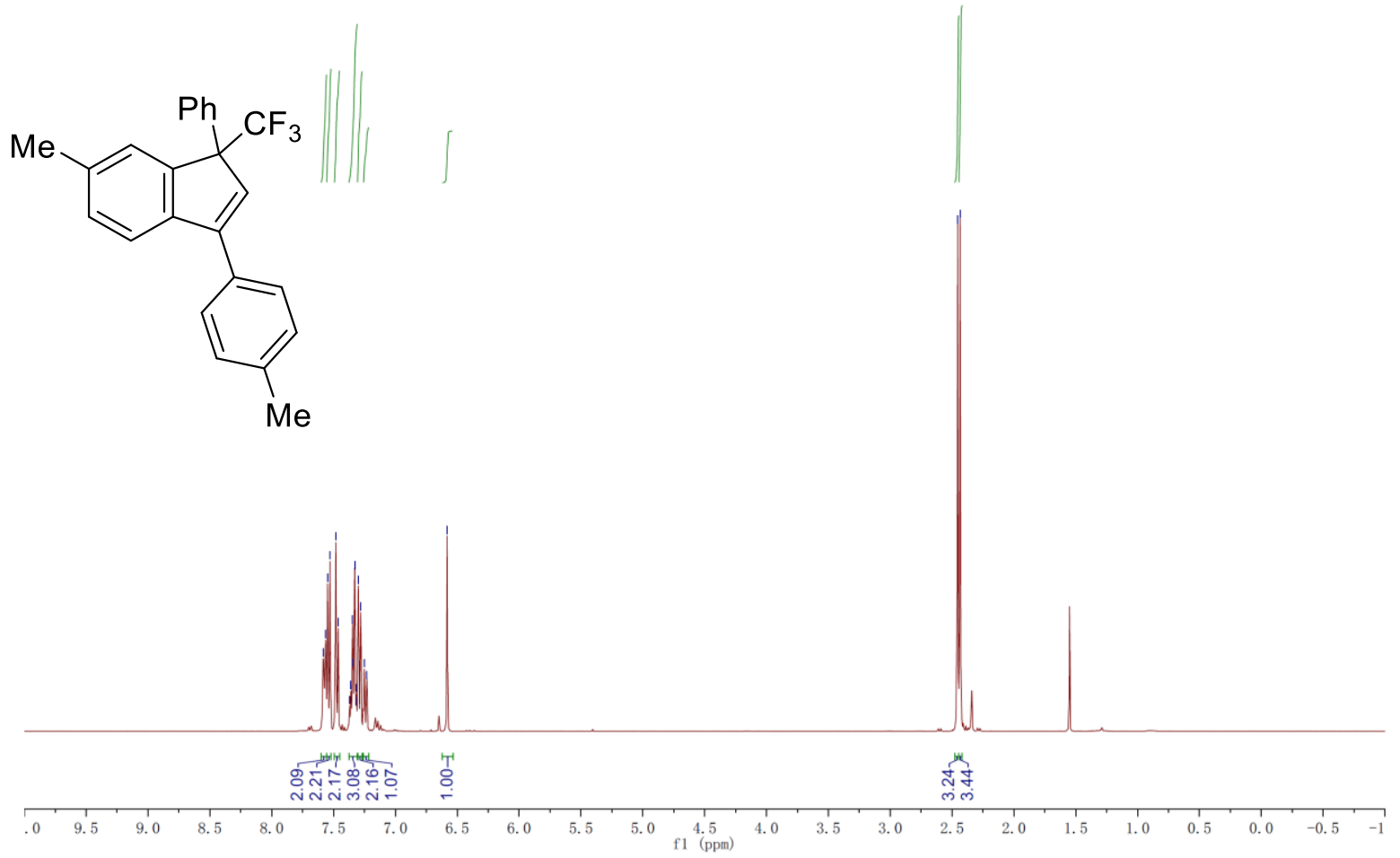

${ }^{13}$ C NMR (101 MHz, Chloroform- $d$ )

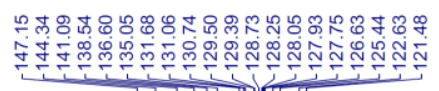

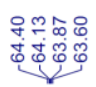

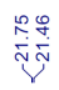
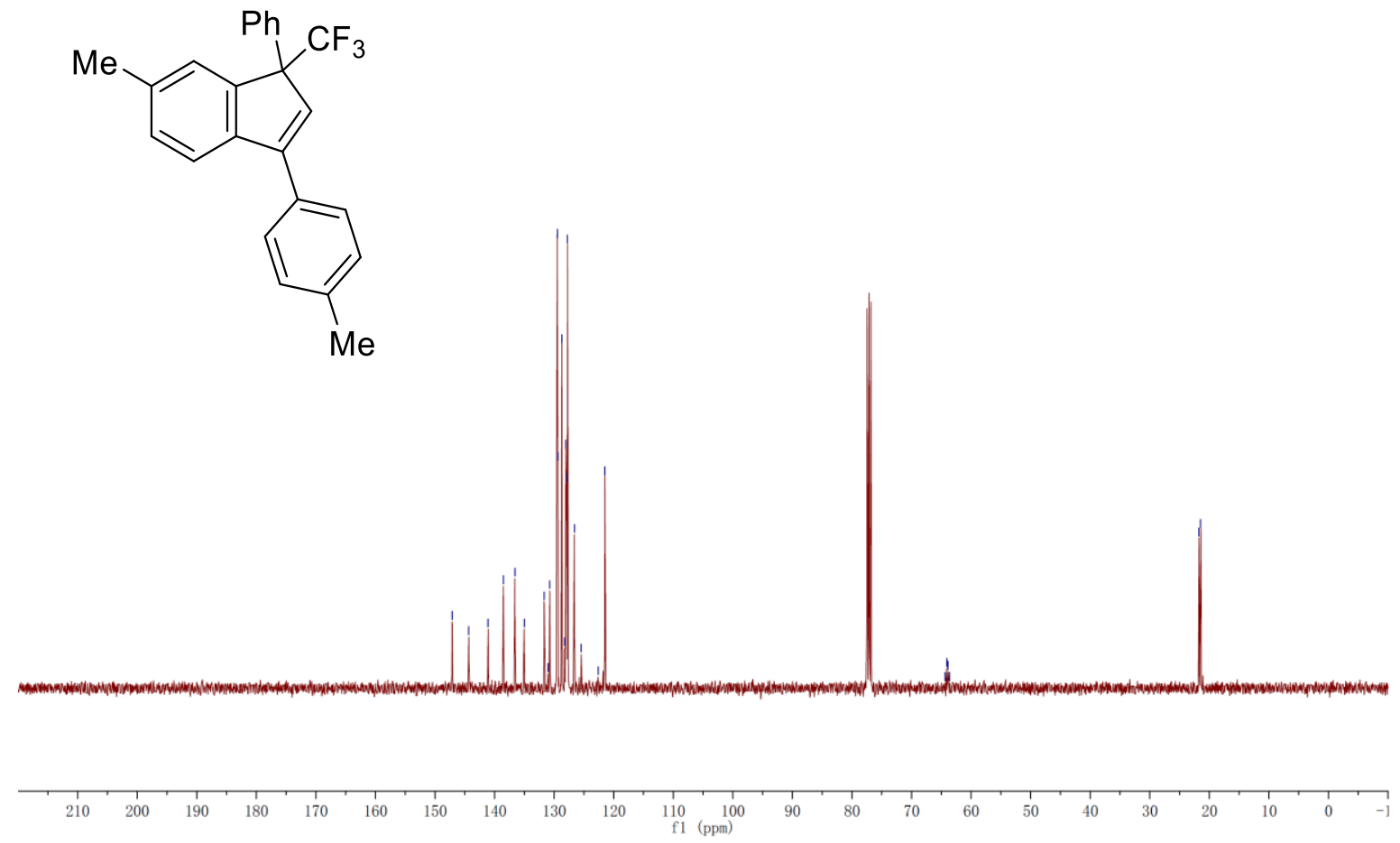

S69 
${ }^{19}$ F NMR (376 MHz, Chloroform- $d$ )

$$
\stackrel{\infty}{\stackrel{\infty}{i}}
$$

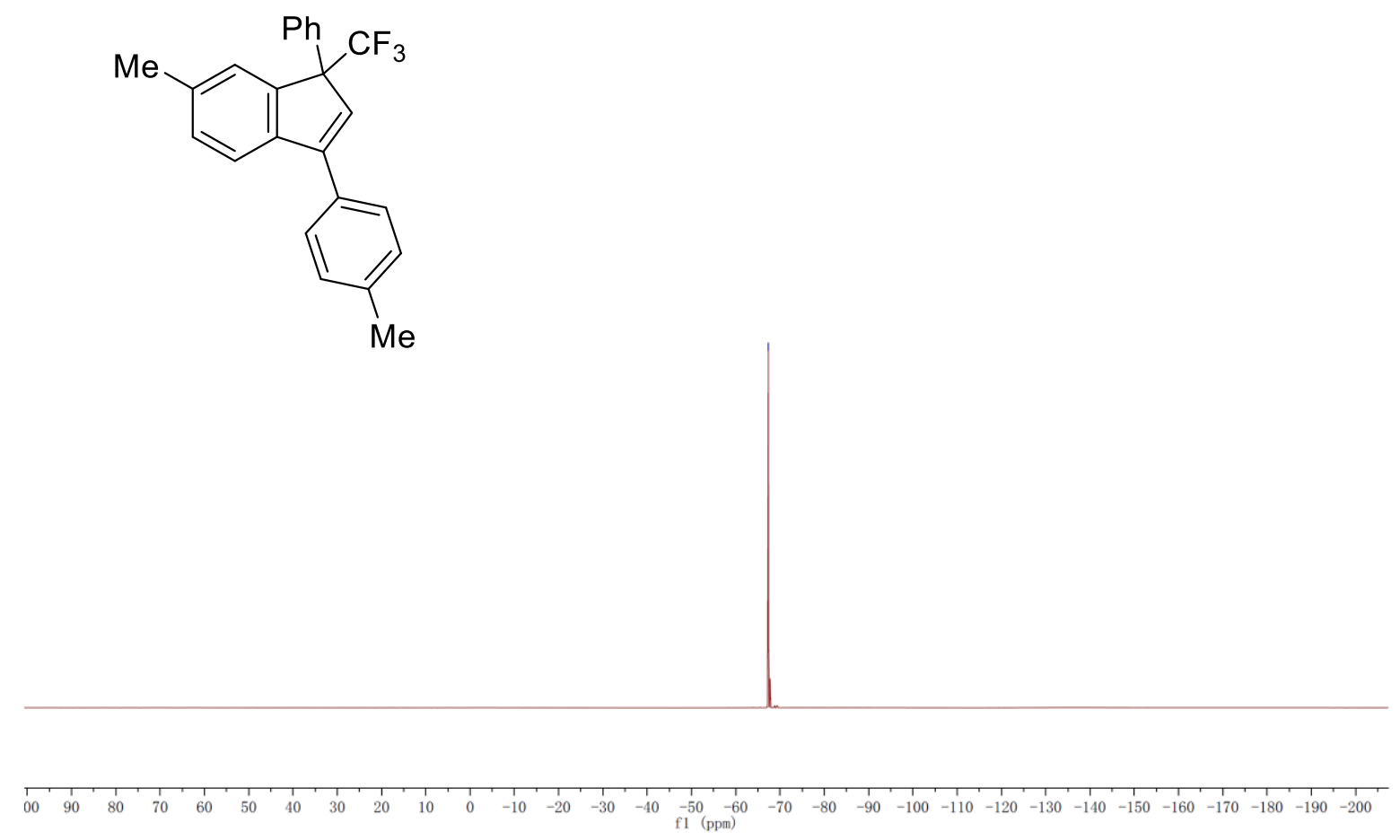


1,1-Di-p-tolyl-3-(trifluoromethyl)- $1 H$-indene (17)

${ }^{1}$ H NMR (600 MHz, Chloroform- $d$ )

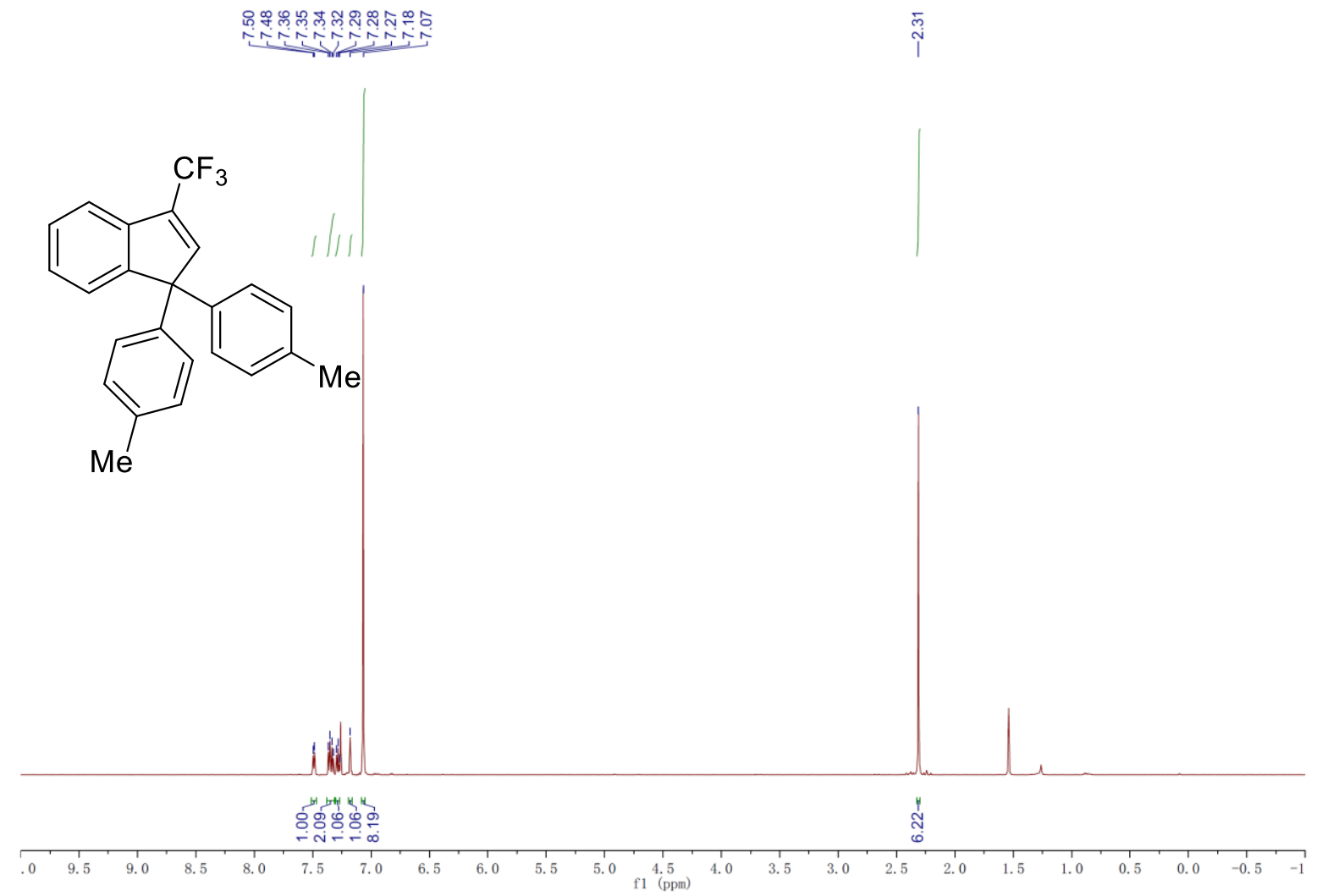

${ }^{13}$ C NMR (151 MHz, Chloroform- $d$ )

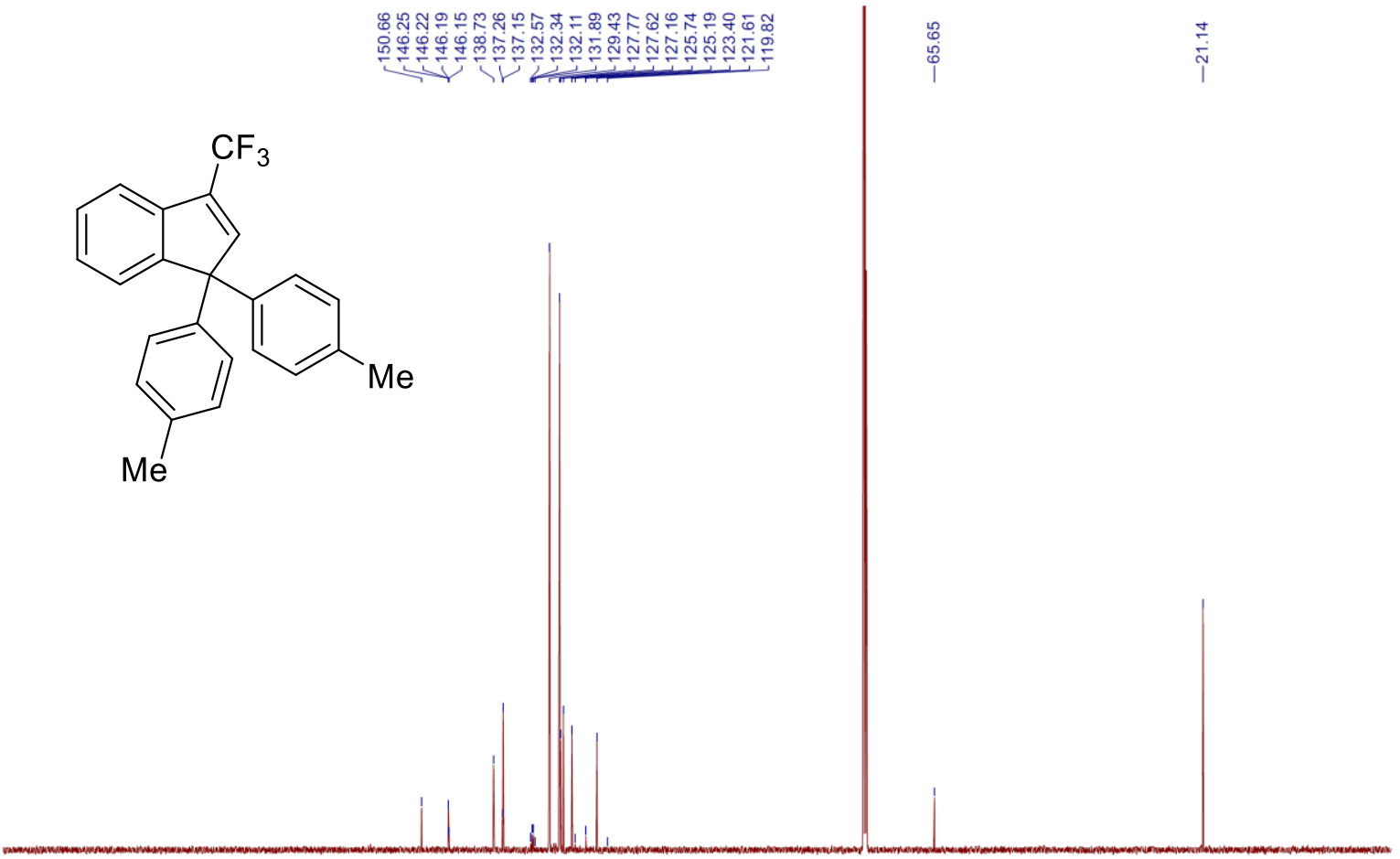

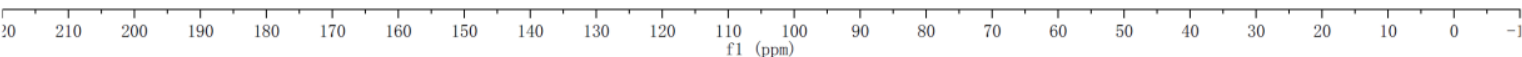


${ }^{19}$ F NMR (565 MHz, Chloroform- $d$ )

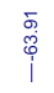

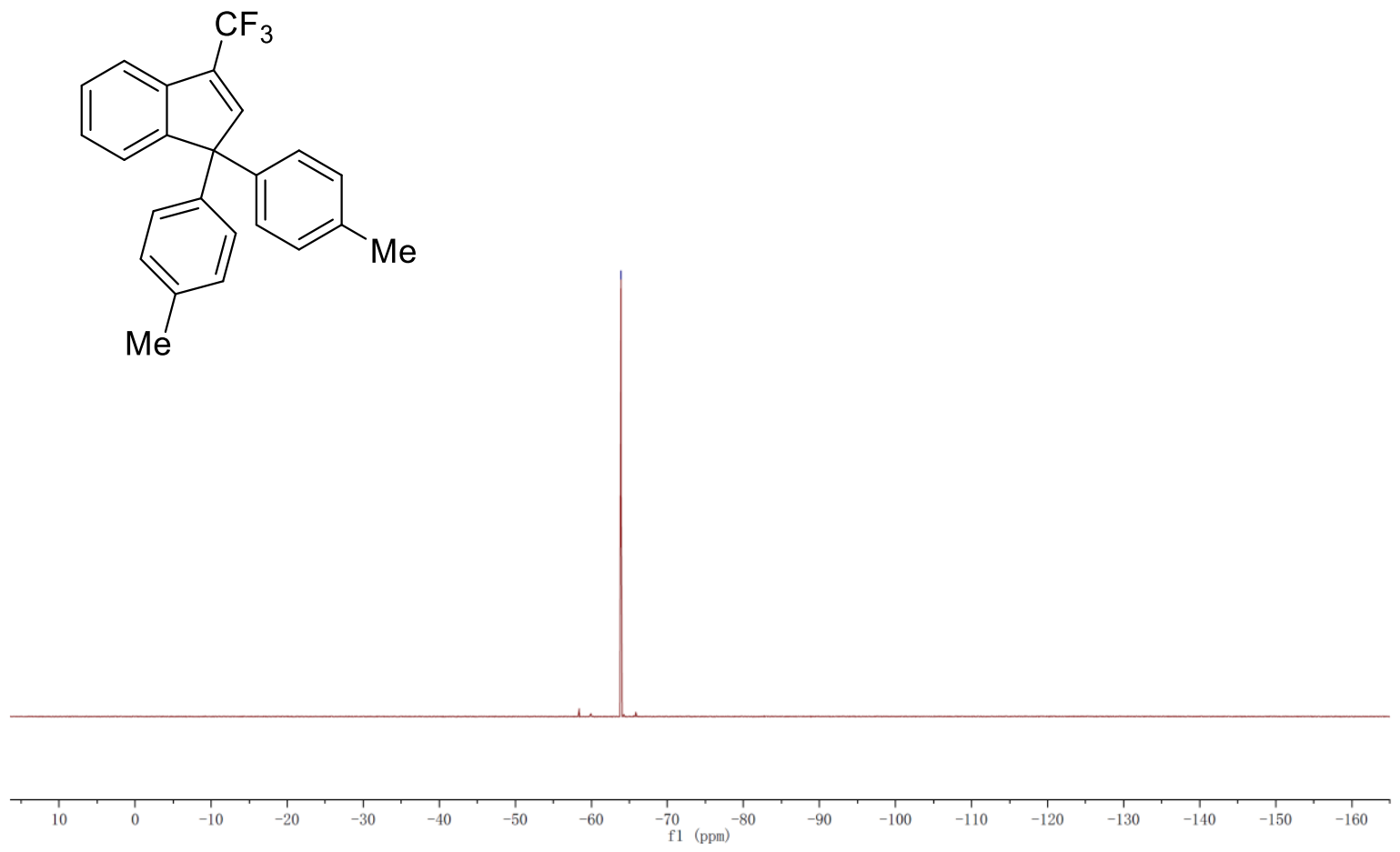

